\title{
DISPERSION OF MASS
} IN OPEN-CHANNEL FLOW

William W. Sayre by

\section{August 1975}


Several departments at Colorado State University have substantial research and graduate programs oriented to hydrology. These Hydrology Papers, therefore, are intended to communicate quickly the current results of this research to specialists interested in these activities. The papers supply most of the background, research data, and results of this work. Shorter versions are usually published in appropriate scientific and professional journals, or presented at national and international scientific and professional meetings and published in the proceedings of these meetings.

The research work leading to this paper was supported by U. S. Geological Survey, Water Resources Division research Program on transport and dispersion Processes in Alluvial channels. Research investigations leading to this paper were made at Colorado State University.

\section{EDITORIAL BOARD}

Dr. Arthur T. Corey, Professor, Agricultural Engineering Department.

Dr. James R. Meiman, Associate Professor, Earth Resources Department.

Dr. Stanley A. Schumm, Professor, Earth Resources Department.

Dr. David A. Woolhiser, Hydraulic Engineer, USDA, ARS, SWC.

Dr. V. Yevjevich, Professor, Civil Engineering Department.

Subscriptions and correspondence to these papers should be addressed to: Secretary of Hydrology Papers, Colorado State University, Fort Collins, Colorado 80521. 


\section{DISPERSION OF MASS IN OPEN-CHANNEL FLOW}

by

William W. Sayre*

HYDROLOGY PAPERS

COLORADO STATE UNIVERSITY

FORT COLLINS, COLORADO

*Professor and Research Engineer, Institute of Hydraulic Research, The University of lowa. Formerly Ph.D. ctıldent, Department of Civil Engineering, Colorado State University. 


\section{ACKNOWLEDGMENTS}

Special thanks are due to Dr. L. V. Baldwin, Dean of Engineering, who both as research adviser and teacher has by his contagious enthusiasm sparked the interest of the writer in turbulent-flow dispersion processes. His helpful advice and encouragement throughout the course of the study has contributed much to this report.

A vote of thanks is extended to Dr. H. R. Bailey, formerly Associate Professor of Mathematics, who bolstered the confidence of the writer during the critical early stages of the numerical analysis when the writer's programs were giving the computer chronic indigestion. Appreciation is also expressed to Drs. M. L. Albertson, D. B. Simons, V. Yevjevich, Professors of Civil Engineering and Dr. R. H. Niemann, Professor of Mathematics, for their review and constructive criticism of the report.

The entire study was.a part of the U. S. Geological Survey, Water Resources Division, research program on transport and dispersion processes in alluvial channels. Dr. F. M. Chang and D. W. Hubbell, research colleagues of the writer in the USGS, participated in the collection and analysis of the experimental data and in many discussions which helped to lay the groundwork for the theoretical phase of the study.

The writer acknowledges the following computing centers for the use without charge of the facilities as listed: The National Center for Atmospheric Research, Boulder, Colorado, for the use of their CDC 6600; the Western Data Processing Center of UCLA for the use of their IBM 7094 via teleprocessing; and the Statistical Laboratory of Colorado State University for the use of their IBM 1620 . 
ABSTRACT . . . . . . . . . . . . . . . . . . . . . . . . . .

I INTRODUCTION . . . . . . . . . . . . . . . . . . . . . . . . 1

II THEORIES OF LONGITUDINAL DISPERSION IN OPEN-CHANNEL FLOW . . . . . . . . . . . . . . . . . . . . . . 3

1. Derivation of the Eulerian Dispersion Equation . . . . . . . . . 3

2. Fickian Diffusion Theory . . . . . . . . . . . . . . . . . 5

3. Diffusion by Continuous Movements .

4. Longitudinal Dispersion by Differential Convection Due to a Velocity Gradient . . . . . . . . . . . . 7

5. Applications of Dispersion Theory in Suspended Sediment Transport . . . . . . . . . . . . . . . 10

6. Reynolds' Analogy for the Equivalence of Mass and Momentum Transfer . . . . . . . . . . . . . . 11

1. Development of the Working Equations. . . . . . . . . . . . 13

2. Analytical Solutions for Simple Cases . . . . . . . . . . . . . . . 14

3. Finite Difference Equations . . . . . . . . . . . . . . . . . 16

4. Statistical Parameters for Describing. . . . . . . . . . . . Numerical Solutions . . . . . . . . . . . . . . . . 18

5. Additional Background Information for Numerical Solutions . . . . . . . . . . . . . . . . . 20

6. Results from Program I. . . . . . . . . . . . . . . . . 22

7. Results from Program II . . . . . . . . . . . . . . 23

a. Results for $\beta=0$. . . . . . . . . . . . . . . . 23

b. Interpretation of results for $\beta=0$ in terms of diffusion by continuous movements. . . . . . . . . 27

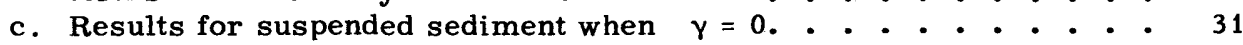

d. Results for suspended sediment when. . . . . . . . . . . . . . $\alpha=1$ and $\gamma>0$...................... 36

e. Results for deposited sediment and composite distributions... . . . . . . . . . . . . . . . 42

f. Estimated longitudinal sediment distribution curves. . . . . . . 46

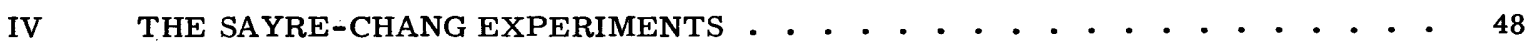

1. Description of Experiments . . . . . . . . . . . . . . . . 48

2. Comparison of Numerical Solutions With Experimental Results. . . . . . . . . . . . . . . . 50

a. Dispersion of fluorescent dye . . . . . . . . . . . . . 50

b. Dispersion of suspended silt . . . . . . . . . . . . . . 52

V SUMMARY AND CONCLUSIONS. . . . . . . . . . . . . . . . . . . . . 60

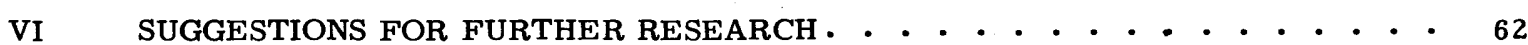

BIBLIOGRAPHY . . . . . . . . . . . . . . . . . . . . . . . 63

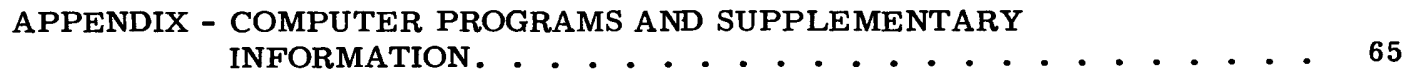




\section{LIST OF FIGURES AND TABLES}

3-1 Definition sketch of variables in finite difference equations . . . . . . . . 16

3-2 Distributions of velocity and eddy diffusivity with respect to depth . . . . 20

3-3 Convective component of longitudinal dispersion coefficient as a function of dispersion time . . . . . . . . . . . . . . . . . . . .

3-4 Variance of longitudinal concentration distribution as a function of dispersion time in flows with a logarithmic velocity distribution for $\kappa=$

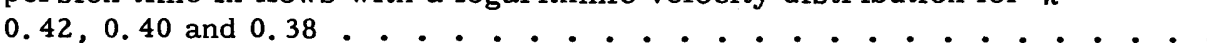

3-5 Convective component of variance of longitudinal concentration distribution as a function of dispersion time . . . . . . . . . . . . . .

3-6 Skew coefficient of longitudinal concentration distribution as a function of dispersion time ..... . . . . . . . . . . . . . . .

3-7 Distribution of properties of longitudinal concentration distribution along a streamline, with respect to depth in a flow with a logarithmic velocity distribution. . . . . . . . . . . . . . . . . . .

3-8 Estimated longitudinal concentration distributions at various dispersion times for $\kappa=0.42$. . . . . . . . . . . . . . . . . . . . 27

3-9 Behavior of the variance at very small dispersion times, $\kappa=0.42$. . .

3-10 Average Lagrangian correlation coefficient as a function of dispersion time. . . . . . . . . . . . . . . . . . . . . .

3-11 Vertical distributions of suspended sediment when $\tau \geq 0.5$ for particles having different terminal fall velocities . . . . . . . . . . . . .

3-12 Fraction of sediment retained in suspension when $\alpha=1$ as a function of dispersion time for particles having different terminal fall velocities. . .

3-13 Mean rate at which particles settle out of suspension as a function of terminal fall velocity. . . . . . . . . . . . . . . . . .

3-14 Mean longitudinal displacement, in a coordinate system moving at the mean flow velocity, of the suspended sediment as a function of dispersion time. . .

3-15 Limiting mean rate of longitudinal displacement, relative to mean flow velocity, of suspended sediment as a function of terminal fall velocity for $\tau \geq 0.5$. . . . . . . . . . . . . . . . . . . .

3-16 Mean longitudinal displacement, from the source, of suspended sediment as a function of dispersion time in a flow with $\bar{U} / U_{\tau}=7$ and $\beta=0.3$. . .

3-17 Variance of longitudinal distribution of suspended sediment as a function of dispersion time . . . . . . . . . . . . . . . . . . . .

3-18 Relative longitudinal dispersion coefficient for suspended sediment as a function of terminal fall velocity . . . . . . . . . . . . . . .

3-19 Intercept on $\sigma_{S}^{2}$ vs. $\tau$ curve for suspended sediment as a function of terminal fall velocity. . . . . . . . . . . . . . . . . . 


\section{LIST OF FIGURES AND TABLES - Continued}

3-20 Skew coefficient for longitudinal distributions of suspended sediment as a function of dispersion time. . . . . . . . . . . . . . . . . 37

3-21 Vertical distribution of suspended sediment at $\tau=3$ with entrainment of deposited particles occurring; $\beta=0.1 . . . . . . . . . . . . . .$.

3-22 Fraction of sediment carried in suspension as a function of dispersion time with entrainment of deposited particles occurring; $\beta=0.1 . . . . . . .$.

3-23 Mean longitudinal displacement, from the source, of the suspended sediment as a function of dispersion time in a flow with $\gamma=0.02$ and $\bar{U} / U_{\tau}=7$. .

3-24 Variance of longitudinal distribution of suspended sediment as a function of dispersion time in a flow with $\gamma=0.02$ and $\overline{\mathrm{U}} / \mathrm{U}_{\tau}=7 . . . . . . \cdot$. .

3-25 Skew coefficient of longitudinal distribution of suspended sediment as a function of dispersion time in a flow with $Y=0.02$ and $\bar{U} / U_{T}=7 . .$. .

3-26 Mean longitudinal displacement, from the source, of the deposited sediment as a function of dispersion time in a flow with $\gamma=0$ and $\bar{U} / U_{\tau}=7 \ldots$. .

3-27 Variance of longitudinal distribution of deposited sediment as a function of

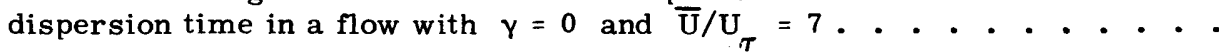

3-28 Skew coefficient of longitudinal distribution of deposited sediment as a function of dispersion time in a flow with $\gamma=0$ and $\bar{U} / U_{\tau}=7$. . . . .

3-29 Mean longitudinal displacement, from the source, of all the sediment as a function of dispersion time in a flow with $Y=0.02$ and $\bar{U} / U_{T}=7 .$. .

3-30 Variance of longitudinal distribution of all the sediment as a function of dispersion time in a flow with $\gamma=0.02$ and $\overline{\mathrm{U}} / \mathrm{U}_{\tau}=7 . . . . . .$. .

3-31 Skew coefficient of longitudinal distribution of all the sediment as a function of dispersion time in a flow with $\gamma=0.02$ and $\overline{\mathrm{U}} / \mathrm{U}_{\tau}=7 . . . . . .$. .

3-32 Esitmated longitudinal distribution of suspended sediment at $\tau=3$ in a

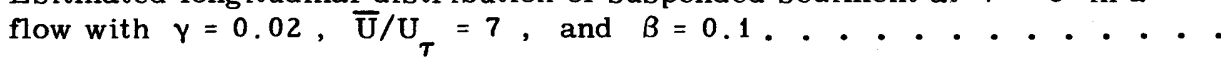

3-33 Estimated longitudinal composite distribution of all the sediment at $\tau=3$ in a flow with $\gamma=0.02, \overline{\mathrm{U}} / \mathrm{U}_{\tau}=7$, and $\beta=0.1 . . . . . . . . .447$

4-1 Velocity distribution data from dispersion experiments. . . . . . . . . . 48

4-2 Continuous and discrete sampling systems used for determining dye and sediment concentrations in flume dispersion experiments. . . . . . . . . 49

4- 3 Typical longitudinal dispersion data for natural silt and glass beads,

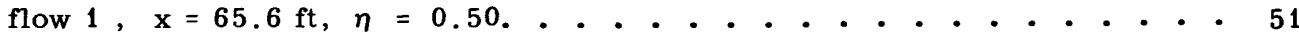

4-4 Fall velocity distribution curves for the sediments used in the experiments .51

4-5 Comparison of experimental and approximate theoretical relative concentration versus time curves, longitudinal dispersion of dye, Run D-1 • • • 53

4-6 Comparison of experimental and approximate theoretical relative concentration versus time curves, longitudinal dispersion of dye, Run D-2 . . . . 53

4-7 Comparison of experimental and approximate theoretical relative concentration versus time curves, longitudinal dispersion of dye, Run D-3 • • • 54 
4-8 Comparison of experimental and approximate theoretical mean travel times, variances and skew coefficients, longitudinal dispersion of dye . . 54

4-9 Comparison of experimental and theoretical mean rates at which particles settle out of suspension. . . . . . . . . . . . . . . . . 55

4-10 Experimental relative concentration versus time curves, longitudinal dispersion of 53-62 $\mu$ glass beads, Run CG-1 . . . . . . . . . . . . 56

4-11 Experimental relative concentration versus time curves, longitudinal dispersion of 53-62 $\mu$ glass beads, Run CG-2 . . . . . . . . . . . . 56

4-12 Experimental relative concentration versus time curves, longitudinal dispersion of 53-62u glass beads, Run CG-3 . . . . . . . . . . . 57

4-13 Comparison of experimental and approximate theoretical recovery ratios, Runs CG-1, CG-2 and CG-3 ................. 57

4-14 Comparison of experimental and approximate theoretical mean travel times, Runs CG-1, CG-2 and CG-3 . . . . . . . . . . . . . . 58

4-15 Comparison of experimental and approximate theoretical variances, Runs CG-1, CG-2 and CG-3 . . . . . . . . . . . . . . . . 59

Table $\quad$ Page

3-1 KEY TO ANALYTICAL SOLUTIONS OF ARIS EQUATIONS . . . . . . . . 14

3-2 RESULTS OF TRIAL AND ERROR MESH-SIZE INVESTIGATIONS . • • . 18

3-3 KEY TO NUMERICAL SOLUTIONS OF ARIS EQUATIONS . • • • • • • . • 21

3-4 APPROXIMATE COMPUTATION TIMES FOR NUMERICAL SOLUTIONS. • • 22

3-5 COMPARISON BETWEEN NUMERICAL AND ANALYTICAL RESULTS FOR CONVECTIVE COMPONENT OF LONGITUDINAL DISPERSION COEFFICIENT AT LARGE DISPERSION TIMES . . . . . . . . . . . 23

3-6 LAGRANGIAN TURBULENCE CHARACTERISTICS FOR FLOWS WITH LOGARITHMIC AND PARABOLIC VELOCITY DISTRIBUTIONS . . . . . . 30

4-1 HYDRAULIC CONDITIONS IN DISPERSION EXPERIMENTS • • . • • • . $\quad$ - 49 


\section{LIST OF SELECTED SYMBOLS}

Symbol

A

$A_{0}$

C

$C_{p}$

D

d

$f_{A}(a ; b)$

$\mathrm{K}_{\mathbf{x}}$

$\mathrm{K}_{\xi}$

$\mathrm{K}_{\mathrm{C}}, \mathrm{K}_{\mathrm{T}}$

$\mathrm{L}_{\mathbf{t}_{\mathbf{i}}}$

$\mathrm{L}_{\boldsymbol{T}}$

$\mathrm{m}_{\mathrm{p}}$

$R_{u_{i}^{\prime}}(\tau)$

$R_{u_{L}^{\prime}}\left(\tau^{\prime}\right)$

S

$S_{S}, S_{W}, S_{T}$

t

$\bar{t}$

$\mathrm{U}$

$\overline{\mathrm{U}}$

$\mathbf{u}^{\prime}$

\section{$\underline{\text { Definition }}$}

Area under concentration vs . time curve

Area under concentration vs. time curve at time $t=0$

Concentration of dispersant at a point in weight per unit volume

p'th moment of longitudinal concentration distribution taken about $\xi=0$

Vertical eddy diffusivity averaged over the depth of flow

Diameter of a sediment particle

Probability density function for the random variable $\mathrm{A}$, with respect to the variable $a$, at the position or time specified by the variable $b$. The variables $A, a$, and $b$, used here for illustrative purposes, are arbitrary

Overall longitudinal dispersion coefficient

$\mathrm{L}^{2} / \mathrm{T}$

Dimensionless overall longitudinal dispersion coefficient

Components of dimensionless overall longitudinal dispersion coefficient due to convection and turbulence, respectively

Lagrangian integral time scale of turbulence for turbulence components in the $i-$ th coordinate direction

$\mathbf{T}$

Dimensionless Lagrangian integral time scale for convective longitudinal component of turbulence

Cross-sectional average of $\mathrm{C}_{\mathrm{p}}$

$\mathrm{F} / \mathrm{L}^{3}$

Lagrangian correlation coefficient which correlates the fluctuating component $u_{i}^{\prime}$ of the velocity of a particle of dispersant at the times $t$ and $t+\tau$

Lagrangian correlation coefficient redefined in terms of the dimensionless variables

Skew coefficient for longitudinal concentration distribution curves

Skew coefficients for the suspended deposited and combined phases, respectively, of the longitudinal distribution of dispersant

Dispersion time

$\mathbf{T}$

Mean time of travel of dispersant over a prescribed distance $\quad T$

Time-averaged local velocity of flow in the $\mathrm{x}$-direction

$\mathrm{L} / \mathrm{T}$

Cross-sectional average of $U$

$\mathrm{L} / \mathrm{T}$

$\mathrm{L} / \mathrm{T}$ 


\section{LIST OF SELECTED SYMBOLS - Continued}

$\mathrm{U}_{\mathbf{S}}$

$\overline{\mathrm{U}}_{\mathbf{S}}$

$\mathrm{U}_{\boldsymbol{T}}$

$\mathrm{V}_{\mathbf{S}}$

W

W

$\mathrm{x}, \mathrm{y}, \mathrm{z}$

$\mathrm{y}_{\mathrm{n}}$

$\alpha$

$\beta$

$\gamma$

$\epsilon$

$\epsilon_{\mathrm{M}}, \epsilon_{\mathrm{T}}$

$\epsilon_{\mathrm{m}}, \epsilon_{\mathrm{y}}, \epsilon_{\mathrm{s}}$

$\eta$

$\lambda$

$\bar{\mu}_{\mathrm{S}}$

$\mu_{X}$

$v_{\mathrm{s}}$
Time-averaged local velocity of a suspended dispersant particle

$\mathrm{L} / \mathrm{T}$

Concentration-weighted cross-sectional average velocity of suspended dispersant

$\mathrm{L} / \mathrm{T}$

Shear velocity in a wide, open channel defined as $\sqrt{\tau / p}$ or $\sqrt{g_{n} \mathrm{~S}_{\mathrm{e}}}$

$\mathrm{L} / \mathrm{T}$

Settling velocity of dispersant in water

$\mathrm{L} / \mathrm{T}$

Amount of dispersant stored per unit area of bed surface

$\mathrm{F} / \mathrm{L}^{2}$

$p^{\prime}$ th moment of longitudinal distribution of $\mathrm{W}$, taken about $\xi=0$

$\mathrm{F} / \mathrm{L}^{2}$

Distance coordinates in the longitudinal, vertical and lateral directions, respectively

L

Normal depth, i.e., depth of flow in a channel with uniform flow

L

Bed absorbency coefficient representing the probability that a particle of dispersant coming into contact with the bed is deposited

Dimensionless fall velocity parameter, defined as $\mathrm{V}_{\mathrm{S}} / \kappa \mathrm{U}_{\boldsymbol{T}}$

Dimensionless entrainment-rate coefficient, defined so that $\gamma W$ is the mean rate of entrainment

Local eddy diffusivity, sometimes called the kinematic eddy viscosity

$\mathrm{L}^{2} / \mathrm{T}$

Molecular and turbulent diffusivities, respectively

$\mathrm{L}^{2} / \mathrm{T}$

Local eddy diffusivities, or turbulent transfer coefficients, for momentum, fluid mass, and sediment particles, respectively

$\mathrm{L}^{2} / \mathrm{T}$

Dimensionless vertical distance coordinate, defined as $y / y_{n}$

von Karman turbulence coefficient if the velocity distribution is logarithmic; otherwise, a proportionality constant

Dimensionless mean rate at which dispersant particles settle out of suspension

Dimensionless mean velocity of flow, defined as $\overline{\mathrm{U}} \mathrm{y}_{\mathbf{n}} / \mathrm{D}$

Dimensionless concentration-weighted average velocity of all suspended dispersant particles

Dimensionless local velocity of flow relative to origin, $\xi=0$, of coordinate system moving at mean dimensionless velocity $\mu$

Dimensionless settling velocity of dispersant in water, defined as $\mathrm{V}_{\mathbf{s}} \mathrm{y}_{\mathbf{n}} / \mathrm{D}$ 
$\xi$

$\bar{\xi}$

$$
\bar{\xi}_{\mathrm{S}}, \bar{\xi}_{\mathrm{W}}, \bar{\xi}_{\mathrm{T}}
$$$$
\sigma_{\mathrm{x}}^{2}, \sigma_{\mathrm{t}}^{2}
$$$$
\sigma_{\xi}^{2}
$$$$
\sigma_{S}^{2}, \sigma_{W}^{2}, \sigma_{T}^{2}
$$

$\boldsymbol{T}$

$\bar{\tau}_{1}$

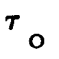

$x$

$\psi$
Dimensionless longitudinal distance coordinate, defined as $(x-\bar{U} t) / y_{n}$

Dimensionless mean longitudinal displacement of dispersant from $\xi=0$

Dimensionless mean longitudinal displacement of dispersant from $\xi=0$ for the suspended, deposited, and combined phases, respectively, of the dispersant

Variances of longitudinal and time concentration distributions, respectively

$\mathrm{L}^{2}, \mathrm{~T}^{2}$

Dimensionless variance of longitudinal concentration distribution of dispersant

Dimensionless variances of longitudinal concentration distributions of suspended, deposited and combined phases, respectively, of the dispersant

Dimensionless dispersion time, defined as $\mathrm{Dt} / \mathrm{y}_{\mathrm{n}}^{2}$

Intercept of the asymptote of the $\sigma_{\xi}^{2}$ vs. $\tau$ relationship on the $\tau$ axis; also the ratio of the first moment of the Lagrangian correlation coefficient, $R_{u_{L}^{\prime}}\left(\tau^{\prime}\right)$ to $L_{\tau}$

Shear stress at the bed; defined as $\gamma_{\mathrm{n}} \mathrm{S}_{\mathrm{e}}$

Weighting function for the local velocity; defined as $(\mathrm{U}-\overline{\mathrm{U}}) / \overline{\mathrm{U}}$

Weighting function for the local eddy diffusivity; defined as $\epsilon_{\mathrm{y}} / \mathrm{D}$ 
The establishment of more reliable criteria for regulating the discharge of contaminants into waterways requires an improved understanding of fluvial transport and dispersion processes. Although the volume of research in this general area has been increasing in recent years, the dispersion of particulate contaminants which behave like silt and fine sand particles has been largely neglected.

In this investigation, the dispersion process for silt particles, in a two-dimensional turbulent shear flow, is formulated in two differential equations, one for particles suspended in the flow and the other for those deposited on the bed. The exchange of particles between the bed and the flow is permitted. Using the Aris moment transformations, the equations are converted to a more tractable system of equations which are solved, mostly by numerical methods with the aid of digital computers, for the zero'th, first, second and third moments of the longitudinal concentration distribution. Various combinations of boundary and other input conditions are imposed and their effect on the results is demonstrated. Included for the sake of comparison is the case of a dispersant consisting of fluid particles having the same properties as the dispersion medium.

The results of the numerical solutions and the results of earlier dispersion experiments with silt particles and fluorescent dyes, although not directly comparable, both exhibit the same general trends. It is shown that the tendency of the silt particles toward deposition and temporary storage on the bed can profoundly affect the longitudinal dispersion process which otherwise resembles the process for fluid particles. The extent of the effect is found to depend primarily on the rate at which deposited particles are re-entrained in the flow, and secondarily on the fall velocity of the particles and flow characteristics in the channel. 


\title{
DISPERSION OF MASS IN OPEN-CHANNEL FLOW
}

\author{
By: William W. Sayre*
}

Chapter I

\section{INTRODUCTION}

With the growing demands on the world's water resources, it becomes increasingly imperative that rational criteria be applied to the problem of allocating available water supplies among all the various competing uses. As requirements grow, the allowable margin for error decreases. All too frequently the information needed for rational decision making is lacking.

One such example has to do with the use of streams and rivers as conveyance channels for the disposal of industrial, agricultural and domestic wastes. Waterways have traditionally performed this function. If pollution is not controlled, however, the availability of water for other equally important uses may be sharply curtailed. In order to control pollution, the release of potentially harmful contaminants into waterways must be regulated so as not to exceed the capacity of the stream to maintain the concentration of contaminants within permissible limits. This requires knowledge of the rate at which the stream system is capable of transporting and dispersing the contaminant in question. In general, transport and dispersion rates depend on the physical and chemical properties of the contaminant, and the physiographic characteristics of and the flow discharge in the stream system. However, the relationships between these factors and the transport and dispersion processes are extremely complex. Because many of them are not adequately understood, presently available criteria for predicting transport and dispersion rates are often unreliable.

With the introduction since World War II of radioactive wastes and the new generation of agricultural pesticides and herbicides, the need for improved criteria has become much more acute. This is largely because (1) the tolerance level for many of these contaminants is several orders of magnitude lower than that for most other pollutants, (2) many of these contaminants are chemically very stable so that they retain their toxicity for long periods of time before yielding to natural decay processes, and (3) many of these contaminants are not removed by conventional water-treatment practices.

For a number of years various federal agencies and university research groups have been investigating dispersion processes in streams. Considerable progress has been achieved in developing criteria for predicting the longitudinal dispersion of substances, such as dissolved or colloidally-suspended contaminants, which behave like particles of the ambient fluid. This class of contaminants is transported at the mean stream velocity, and dispersed longitudinally by the combined action of turbulent diffusion and differential convection due to the variation of velocity with respect to position in the cross section. Research on the transport and longitudinal dispersion of contaminants which behave like bed-material sediment particles has also yielded useful results. Bed material particles move in a sequence of discrete steps of random length that are separated in time by rest periods of random duration. Hence, they are transported at rates which are much less, frequently by several orders of magnitude, than the rates for dissolved or colloidally-suspended materials. Between these extremes is the class of contaminated particles which, like silt and fine sand, are transported mainly in suspension. While suspended, they behave very much like fluid or collodially-suspended particles except that they tend to settle and eventually be deposited on the bed. There they behave like bed-material particles until they are re-entrained in the flow.

This study is the second phase of a two-part investigation, conducted by the Water Resources Division of the U.S. Geological Survey, which is concerned with the intermediate class of dispersants. In the first phase, Sayre and Chang (1966), in a series of flume experiments, compared the longitudinal dispersion of suspended silt particles with the longitudinal dispersion of fluorescent dyes. The principal objective of the second phase is to make the same comparison from a theoretical viewpoint.

This report begins with a review of longitudinal dispersion and other pertinent theories. The dispersion process for silt particles is then formulated in two differential equations, one for particles suspended in the flow and the other for those deposited on the bed. Using the moment transformations of Aris (1956), the equations are then converted to a more tractable system of equations which are solved, mostly by numerical methods with the aid of digital computers, for the zero'th, first, second and third moments of the longitudinal concentration distribution. Various combinations of boundary and other input conditions are imposed, and the manner in which they affect the results is demonstrated. The results of

* Research Hydraulic Engineer, Water Resources Division, U.S. Geological Survey, Fort Collins, Colorado 
the numerical solutions are then compared with the results of the Sayre-Chang experiments.

The investigation is restricted to a consideration of dispersion in a uniform, two-dimensional, open-channel, turbulent shear flow. The initial condition is restricted to an instantaneous plane source, uniformly distributed over the flow cross section.
In addition, it is assumed that local values of the vertical eddy diffusivities for the transfer of mass and momentum are equal. Also, the mean fall velocity of silt particles is assumed to be the same under both turbulent and quiescent conditions. Finally, the term dispersant, as used in this study, means the substance which is being dispersed. 


\section{THEORIES OF LONGITUDINAL DISPERSION IN OPEN-CHANNEL FLOW}

The processes by which dissolved matter and suspended particles are dispersed in open-channel flows have been subjected to considerable theoretical analysis in recent years. Due to a number of complex factors that are almost invariably associated with open-channel flow such as turbulence, velocity gradients in the vertical and horizontal directions, and secondary flows, theoretical treatments have been only partially successful. No theory, not even for the relatively simple case of uniform flow in a straight channel of constant cross section, has been formulated which accounts adequately for all of the above factors.

\section{Derivation of the Eulerian Dispersion Equation}

One of the most direct theoretical approaches to the dispersion process is based on the principle of conservation of mass. Consider the arbitrary control volume illustrated below

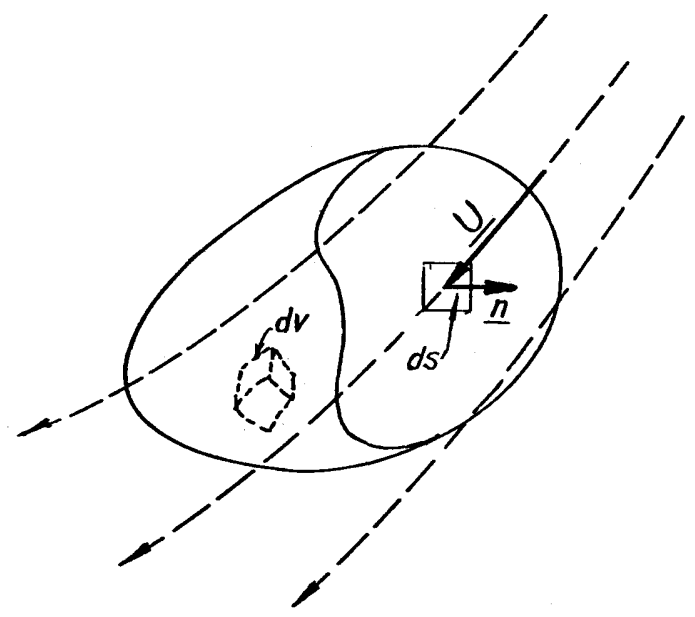

of which $d v$ is a volume element, ds is an element of surface area, where $\underline{n}$ is a unit vector normal to the surface, and $\underline{U}$ defines the velocity field. The rate of change of weight, $W$, of dispersant in the control volume is

$$
\begin{aligned}
\frac{d W}{d t} & =\begin{array}{l}
\text { Rate of change } \\
\text { of weight due to } \\
\text { convection }
\end{array}+\begin{array}{l}
\text { Rate of change } \\
\text { of weight due to } \\
\text { diffusion }
\end{array} \\
& =-\int_{S} \underline{C} \underline{U} \cdot \underline{\text { nds }}+\int_{S} \epsilon \nabla C \cdot \underline{\text { nds }},
\end{aligned}
$$

where $C$ is the concentration (weight per unit volume) of dispersant and $\epsilon$ is a diffusion coefficient, assumed here to be a constant. Noting that

$$
\frac{d W}{d t}=\frac{\partial}{\partial t} \int_{V} C d v=\int_{V} \frac{\partial C}{\partial t} d v,
$$

and employing the divergence theorem whereby

$$
\int_{S} C \underline{U} \cdot \underline{n d s}=\int_{V} \nabla \cdot(C \underline{U}) d v
$$

and

$$
\int_{S} \epsilon \nabla C \cdot \underline{n d s}=\int_{V} \epsilon \nabla^{2} C d v,
$$

the statement for the conservation of mass of the dispersant becomes

$$
\frac{\partial C}{\partial t}=-\nabla \cdot(C \underline{U})+\epsilon \nabla^{2} C
$$

For incompressible flow where $\nabla . \underline{U}=0$, this equation reduces further to

$$
\frac{\partial \mathrm{C}}{\partial \mathrm{t}}+\underline{U} \cdot \nabla \mathrm{C}=\epsilon \nabla^{2} \mathrm{C}
$$

or in tensor form to

$$
\frac{\partial C}{\partial t}+U_{i} \frac{\partial C}{\partial x_{i}}=\epsilon \frac{\partial^{2} C}{\partial x_{i} \partial x_{i}},
$$

where $x_{i}$ represents distance and $i=1,2,3$ indicates direction in a rectangular coordinate system. Equations 2-1 and 2-1a are standard forms of the Eulerian diffusion equation in a convecting flow field. They are called Eulerian because attention is focused on the changes occurring as the fluid passes through a stationary control volume. In laminar flow, where $\epsilon=\epsilon_{M}$ is the coefficient of molecular diffusion, equation 2-1, together with the appropriate boundary and initial conditions, gives an exact description of the dispersion process. Significant theoretical work on longitudinal dispersion in tubes with laminar flow has been reported by Taylor (1953, 1954b), Aris (1956), Ananthakrishnan, Gill and Barduhn (1965), and Bailey and Gogarty (1962).

The dispersion process in turbulent flow is considerably more complicated. However, by use of the Reynolds averaging procedure, equation 2-1 has 
been extended to include the case of dispersion in turbulent flow. The instantaneous concentrations and velocities are resolved into the sum of a time averaged and a fluctuating component

$$
\mathrm{C}=\overline{\mathrm{C}}+\mathrm{c}^{\prime}, \quad \mathrm{U}_{\mathrm{i}}=\overline{\mathrm{U}}_{\mathrm{i}}+\mathrm{u}_{\mathrm{i}}^{\prime}
$$

where

$$
\bar{C}=\frac{1}{T} \int_{t}^{t+T} C d t, \quad \bar{U}_{i}=\frac{1}{T} \int_{t}^{t+T} U_{i} d t
$$

As shown conceptually in the illustration below, the averaging period $T$ is supposed to be sufficiently long to permit convergence of the averages of the primed quantities to zero, yet not so long as to significantly damp the variation of $\bar{C}$ with $t$.

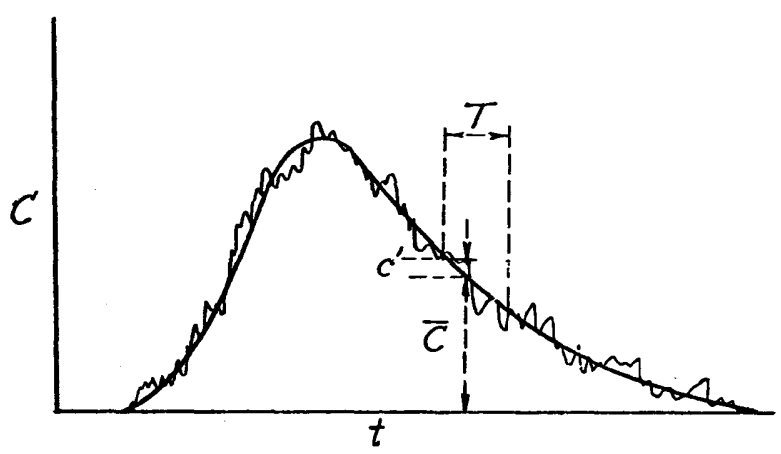

Making the appropriate substitution in equation 2-1, and averaging the resulting equation over $T$ gives

$$
\frac{\partial \bar{C}}{\partial t}+\bar{U}_{i} \frac{\partial \bar{C}}{\partial x_{i}}=-\frac{\partial}{\partial x_{i}} \overline{c^{\prime} u_{i}^{\prime}}+\epsilon_{M} \frac{\partial^{2} \bar{C}}{\partial x_{i} \partial x_{i}}
$$

A coefficient of turbulent diffusion,

$$
\epsilon_{T_{i j}}=\epsilon_{T_{i j}}\left(x_{1}, x_{2}, x_{3}\right)
$$

may be defined as

$$
\epsilon_{T_{i j}} \frac{\partial \bar{C}}{\partial x_{j}} \equiv-\overline{c^{\prime} u_{i}^{\prime}}
$$

The implications of equation 2-3 are discussed in detail in section II- 6 at the end of this chapter. Assuming that the processes of molecular and turbulent diffusion are independent and therefore additive, Mickelsen (1960), it can be concluded that

$$
\epsilon_{i j}\left(x_{1}, x_{2}, x_{3}\right)=\epsilon_{T_{i j}}+\epsilon_{M}
$$

Incorporating equations $2-3$ and 2-4, and dropping the overbars which are no longer needed to denote a time average, equation $2-2$ becomes

$$
\frac{\partial C}{\partial t}+U_{i} \frac{\partial C}{\partial x_{i}}=\frac{\partial}{\partial x_{i}}\left|\epsilon_{i j} \frac{\partial C}{\partial x_{j}}\right|
$$

which is similar in form to equation $2-1$ except that $\epsilon_{i j}$, commonly called the diffusion tensor, is now a function of position in the flow field. Taking the axes of the coordinate system as the principal axes of the diffusion tensor, in which case $\epsilon_{i j}=0$ for $i \neq j$, and replacing $\epsilon_{i j}$ by the scalar $\epsilon_{i}$ for $i=j$, equation 2-5 reduces further to

$$
\frac{\partial C}{\partial t}+U_{i} \frac{\partial C}{\partial x_{i}}=\frac{\partial}{\partial x_{i}}\left|\epsilon_{i} \frac{\partial C}{\partial x_{i}}\right|
$$

With regard to equation 2-4, the question of whether molecular and turbulent diffusion actually are independent processes is mainly academic because in ordinary open-channel flows $\epsilon_{T}$ is several orders of magnitude larger than $\epsilon_{\mathbf{M}}$.

Up to this point the derivation has been quite general. The assumptions which have been made are generally believed to be physically sound and there is no particular reason to doubt that equation $2-5 \mathrm{a}$ is indeed capable of describing dispersion processes in open channels quite accurately. Also, the derivation applies equally well whether the dispersant is, (1) in solution form, or (2) in the form of suspended particles provided that the percentage of volume actually occupied by the particles is negligible. However, in the latter case $U_{i}$ must be considered as the velocity at which particles are convected, and $\epsilon_{i}$ as a diffusion coefficient for the particles. The main obstacles to progress in this approach to dispersion theory have been the lack of a reliable theory which relates the spatial variation of $\epsilon_{i}$ to flow and boundary conditions, and the mathematical difficulties in solving equation 2-5a for a variable $\epsilon_{i}$ which would remain formidable even if the nature of the variation were better known.

Consider now the dispersion process for a neutrally buoyant dispersant under conditions of uniform flow in a straight channel of constant cross section. Choosing a coordinate system such that the indices $i=1,2,3$ indicate respectively the direction of flow, the direction normal to the channel bed and the transverse direction, it is evident that $\mathrm{U}_{2}=\mathrm{U}_{3}=0$. Furthermore, it is to be expected that although $\epsilon_{i}$ may vary with $x_{2}$ and $x_{3}$, it should not vary with $x_{1}$. Equation 2-5a may now be rewritten

$\frac{\partial C}{\partial t}+U_{1} \frac{\partial C}{\partial x_{1}}=\epsilon_{1} \frac{\partial^{2} C}{\partial x_{1}^{2}}+\frac{\partial}{\partial x_{2}}\left|\epsilon_{2} \frac{\partial C}{\partial x_{2}}\right|+\frac{\partial}{\partial x_{3}}\left|\epsilon_{3} \frac{\partial C}{\partial x_{3}}\right|$

or after switching to the more conventional form of Cartesian notation in which $x_{1}, x_{2}$ and $x_{3}$ are replaced by $x, y$ and $z ; U_{1}, U_{2}$ and $U_{3}$ by $U, V$ and $W$; and $\epsilon_{1}, \epsilon_{2}$, and $\epsilon_{3}$ by $\epsilon_{x}, \epsilon_{y}$ and $\epsilon_{z}$, 
$\frac{\partial C}{\partial t}+U \frac{\partial C}{\partial x}=\epsilon_{x} \frac{\partial^{2} C}{\partial x^{2}}+\frac{\partial}{\partial y}\left|\epsilon_{y} \frac{\partial C}{\partial y}\right|+\frac{\partial}{\partial z}\left|\epsilon_{z} \frac{\partial C}{\partial z}\right|$

Theoretical investigations of dispersion in open-channel flow have been devoted mainly to (1) establishing a theoretical basis for predicting $\epsilon_{\mathrm{x}}, \epsilon_{\mathrm{y}}$ and $\epsilon_{\mathrm{z}}$, (2) the analysis of certain limiting cases for which solutions to equation 2-6 can be obtained, (3) the transformation of equation 2-6 into forms which are more amenable to solution, and beginning only recently, (4) the numerical solutions of equation 2-6 by finite difference methods using computers.

The most promising approaches have been based on (1) the semi-empirical Fickian diffusion theory, (2) the theory of diffusion by continuous movements, (3) the theory of longitudinal dispersion by differential convection due to a velocity gradient, and (4) dispersion theory as applied to the transport of suspended sediment. Because all of these theories have a direct bearing on the work reported in this report, a review of the pertinent results and limitations is appropriate here.

\section{Fickian Diffusion Theory}

In applying the Fickian diffusion theory to dispersion processes in turbulent open-channel flow, an exact analogy with the molecular diffusion process is assumed. Although the analogy is at best crude, in that unrealistic restrictions are imposed on the descriptions of the flow, it leads to useful results in some cases. Usually the coefficients $\epsilon_{\mathrm{x}}, \epsilon_{\mathrm{y}}$ and $\epsilon_{\mathrm{z}}$ are assumed to be constants which we shall call $\mathrm{K}_{\mathrm{x}}, \mathrm{K}_{\mathrm{y}}$ and $K_{z}$. Also, the velocity $U$ everywhere in the flow field is assumed to equal the average velocity in the cross section, $\bar{U}$. With these assumptions, equation $2-6 \mathrm{a}$ becomes

$\frac{\partial C}{\partial t}+\bar{U} \frac{\partial C}{\partial x}=K K_{x} \frac{\partial^{2} C}{\partial x^{2}}+K \frac{\partial^{2} C}{\partial y^{2}}+K \frac{\partial^{2} C}{\partial z^{2}}$.

The convection term, $\overline{\mathrm{U}} \frac{\partial \mathrm{C}}{\partial \mathrm{x}}$, means that dispersion is occurring in a frame of reference which is moving at a velocity $\bar{U}$ in the $x$ direction.

Equation 2-7 has received considerable attention, and solutions for a great variety of initial and boundary conditions have been published. Among the best references are Crank (1956), and Carslaw and Jaeger (1959).

The case of primary interest here is for longitudinal dispersion from an instantaneous plane source which is distributed uniformly over the cross section. With this initial condition and the restrictions on equation 2-7, the problem becomes one-dimensional and equation $2-7$ reduces to

$$
\frac{\partial \bar{C}}{\partial t}+\bar{U} \frac{\partial \bar{C}}{\partial x}=K_{x} \frac{\partial^{2} \bar{C}}{\partial x^{2}}
$$

where $\bar{C}(x, t)$ is the concentration averaged over the cross section. The solution of equation 2-8 for the indicated condition is

$$
\frac{\bar{C}(x, t)}{\int_{-\infty}^{\infty} \bar{C} d x}=f(x ; t)=\frac{1}{2 \sqrt{\pi K_{x}^{t}}} e^{-\frac{(x-\bar{U} t)^{2}}{4 K_{x} t}} .
$$

The function $f(x ; t)$ is the probability density function of the normal probability law with mean $\bar{x}=\bar{U} t$ and variance $\sigma_{x}^{2}=2 K_{x} t$. For a rectangular channel of width $B$ with a depth of flow, $y_{n}$, and where the concentration of dispersant is defined as a weight per unit volume, the normalizing factor (initial source strength) is

$$
\int_{-\infty}^{\infty} \bar{C} d x=\frac{w}{B y_{n}}
$$

where $\mathrm{W}$ is the total weight of dispersant. In comparing the theory with experimental results it is usually more convenient to consider $\bar{C}$ as a function of $t$ with $x$ as a parameter. Then equation 2-9 becomes

$$
\frac{\bar{C}(t, x)}{\int_{0}^{\infty} \bar{C} d t}=f(t ; x)=\frac{\bar{U}}{2 \sqrt{\pi K t}} e^{-\frac{(x-\bar{U} t)^{2}}{4 K x^{t}}}
$$

in which the normalizing factor is now

$$
\int_{0}^{\infty} \bar{C} d t=\frac{W}{B y_{n} \bar{U}} \text {. }
$$

The function $f(t ; x)$ is the probability density function for the distribution of dispersant flux with respect to time, $t$, at a fixed position, $x$. The mean and variance of $f(t ; x)$ as given by Yotsukura (1963) are

$$
\bar{t}=\int_{0}^{\infty} t f(t ; x) d t=\frac{x}{\bar{U}}+\frac{2 K x}{\bar{U}^{2}}
$$

and

$$
\sigma_{t}^{2}=\int_{0}^{\infty}(t-\bar{t})^{2} f(t ; x) d t=\frac{2 K_{x} x}{\bar{U}^{3}}+8\left(\frac{K_{x}}{\bar{U}^{2}}\right)^{2} .
$$

Experimental evidence, Sayre and Chang(1966) Yotsukura, Smoot, and Cahal (1964), Glover (1964), Godfrey and Frederick (1963), indicates that the Fickian diffusion theory provides at best a rough kinematic description of the longitudinal dispersion process 
in open channels. In general, the agreement between the Fickian theory and experimental observations is poor in the early stages of the process, but tends to improve with increasing dispersion time or distance from the source. Thus, in some cases, for example, dispersion in a uniform channel with straight alignment, equations 2-9 and 2-10 are useful as asymptotic solutions for large values of $t$ and $x$, respectively.

An important property of the Fickian diffusion process is that the variance of the concentration distribution increases linearly with time. This property is often used for evaluating the longitudinal dispersion coefficient from the variances calculated from a set of observed concentration versus distance or concentration versus time curves by means of the relation ships

$$
K_{x}=\frac{1}{2} \frac{d \sigma_{x}^{2}}{d t}
$$

or

$$
K_{x}=\frac{\bar{U}^{3}}{2} \frac{d \sigma_{t}^{2}}{d x}
$$

Fischer (1966) has shown that both of these relationships can be derived directly from equation 2-8, and moreover that they are independent of the initial distribution of dispersant.

Actually, in view of the glaring discrepancies between the assumed model and the actual nature of the process, it is remarkable that the one-dimensional Fickian diffusion theory has any applicability whatsoever to longitudinal dispersion in turbulent shear flows. From comparing equation 2-6a with equation 2-8 and letting

$$
\mathbf{U}=\overline{\mathrm{U}}+\mathrm{u}(\mathrm{y}, \mathrm{z}),
$$

it seems that at large dispersion times the group of terms

$-u(y, z) \frac{\partial C}{\partial x}+\epsilon_{x} \frac{\partial^{2} C}{\partial x^{2}}+\frac{\partial}{\partial y}\left(\epsilon_{y} \frac{\partial C}{\partial y}\right)+\frac{\partial}{\partial z}\left(\epsilon_{z} \frac{\partial C}{\partial z}\right)$

interact in such a way that they behave collectively like a longitudinal diffusion term

$$
K_{x} \frac{\partial^{2} \bar{C}}{\partial x^{2}}
$$

\section{Diffusion by Continuous Movements}

The theory of diffusion by continuous movements, Taylor (1921), like the Fickian theory is restricted to giving a kinematic description of dispersion, but the description is much more realistic because it is based on the actual turbulence properties of the flow. The theory was originally formulated for the case of one-dimensional dispersion in a turbulence field which is spatially homogeneous and stationary in time.

The discussion heretofore has been geared to an Eulerian description of motion. Taylor's theory, in contrast, is Lagrangian in that it is based on a description of the motion of discrete fluid particles.

Consider the dispersion of a group of fluid particles which are initially concentrated at the origin. Let

$$
x_{i}=\int_{0}^{t} u_{i}^{\prime}\left(t^{\prime}\right) d t^{\prime}
$$

be the displacement from the origin in the $i$ direction of a particle at time $t$, where $u_{i}(t)$ is the instantaneous velocity of the particle in the $i$ direction relative to the origin. Then, defining $\sigma_{i}^{2}(t)$ as the variance of the distribution of particles at time $t$,

$$
\begin{aligned}
& \frac{1}{2} \frac{d \sigma_{i}^{2}(t)}{d t}=\frac{1}{2} \frac{d}{d t} \overline{x_{i}^{2}}=\overline{x_{i} \frac{d x_{i}}{d t}}=\overline{x_{i} u_{i}^{\prime}(t)} \\
& =\overline{u_{i}^{\prime}(t) \int_{0}^{t} u_{i}^{\prime}\left(t^{\prime}\right) d t^{\prime}}=\int_{0}^{t} \overline{u_{i}^{\prime}(t) u_{i}^{\prime}\left(t^{\prime}\right) d t^{\prime}}
\end{aligned}
$$

Introducing the Lagrangian correlation coefficient,

$$
R_{u_{i}^{\prime}}(\tau) \equiv \frac{\overline{u_{i}^{\prime}(t) u_{i}^{\prime}\left(t^{\prime}\right)}}{\overline{u_{i}^{\prime 2}}},
$$

where

$$
\begin{gathered}
\tau=t-t^{\prime} \\
\frac{1}{2} \frac{d \sigma_{i}^{2}(t)}{d t}=\overline{u_{i}^{\prime 2}} \int_{0}^{t} R_{u_{i}^{\prime}}(\tau) d \tau
\end{gathered}
$$

and

$$
\sigma_{i}^{2}(t)=2 \overline{u_{i}^{\prime 2}} \int_{0}^{t} \int_{0}^{t^{\prime}} R_{u_{i}^{\prime}}(\tau) d \tau d t^{\prime}
$$

which after integration by parts becomes

$$
\sigma_{i}^{2}(t)=2 \overline{u_{i}^{\prime 2}} \int_{0}^{t}(t-\tau) R_{u_{i}^{\prime}}(\tau) d \tau
$$

Equation 2-15 is the main result of Taylor's theory. It describes one-dimensional dispersion about the origin, or in the case where the entire velocity field is being convected at a uniform velocity $\bar{U}_{i}$, it describes the dispersion about a plane which is moving at that velocity. In general, $\sigma_{i}^{2}(t)$ depends on the functional form of $R_{u_{i}^{\prime}}(\tau)$ which, like all Lagrangian turbulence properties, is difficult to determine and is therefore usually not known. However, useful information can be obtained from equation 2-15 for the limiting cases of (1) very small dispersion 
times where

$$
\operatorname{Lim}_{\tau \rightarrow 0} R_{i}(\tau)=1
$$

and equation $2-15$ reduces to

$$
\sigma_{i}^{2}(t) \approx \overline{u_{i}^{\prime 2}} t^{2}
$$

and (2) large dispersion times where

$$
\operatorname{Lim}_{\tau \rightarrow \infty} R_{u_{i}}(\tau)=0
$$

and equation $2-15$ reduces to

$$
\sigma_{i}^{2}(t) \approx 2 \overline{u_{i}^{\prime 2}} L_{t_{i}} t-2 \overline{u_{i}^{\prime 2}} \int_{0}^{\infty} \tau R_{u_{i}^{\prime}}(\tau) d \tau
$$

where

$$
L_{t_{i}} \equiv \int_{0}^{\infty} R_{u_{i}^{\prime}}(\tau) d \tau
$$

is the Lagrangian integral time scale of turbulence. Given the homogeneity and stationarity of the turbulence, the second term on the right of equation 2-17 is a constant so that as $t$ becomes very large

$$
\sigma_{i}^{2}(t) \approx 2 \overline{u_{i}^{\prime 2}} L_{t_{i}} t
$$

This is equivalent to the variance given by the Fickian theory when the substitution

is made.

$$
K_{i}=\overline{u_{i}^{\prime 2}} L_{t_{i}}
$$

Equation 2-15 was originally derived to describe dispersion in a homogeneous turbulence field. Thus, at first glance it appears not at all applicable to dispersion in turbulent shear flows where the statistical properties of the turbulence, in general, vary with the distance from the boundary. However, Orlob $(1958,1961)$ showed that planes which are parallel to the bed in wide channels with uniform flow, for example the water surface, can indeed satisfy the criteria for a homogeneous turbulence field. Also, it was pointed out by. Batchelor and Townsend (1956) for steady uniform flows confined by boundaries that, with respect to the longitudinal direction, the turbulence structure is axially homogeneous and that the instantaneous velocity of a fluid particle is necessarily a stationary random function of time provided the observation period is sufficiently long for the particle to have wandered all over the cross section. This is to say that $U_{x}=\bar{U}_{x}+u_{x}^{\prime}$, where $\bar{U}_{x}$ is the discharge velocity (Q/A), is independent of $x$ and becomes a stationary random function of time even though $u_{x}^{\prime}$ is a function of position in the cross section also. Therefore, the theory of diffusion by continuous movements does apply to longitudinal dispersion in any uniform channel so long as the flow is axially homogeneous and the observation period is sufficiently long. A very important aspect of this conclusion is that it gives theoretical support, if not definite proof, to the applicability of the Fickian diffusion theory to the dispersion process in open channels at large dispersion times.

\section{Longitudinal Dispersion by Differential Convection} Due to a Velocity Gradient

In this class of theories equation 2-6a, the basic Eulerian dispersion equation for turbulent flow, is converted by assumptions and transformations into more workable forms from which a quantitative des cription of the most important features of the longitudinal dispersion process can be deduced. A basic premise which is borne out by these theories is that the rate of spread of dispersant in the longitudinal direction due to turbulence is very small in comparison to the rate of spread due to differential convection caused by velocity variation in the cross section.

To facilitate comparisons, the notation and dimensionless parameters introduced by Aris (1956) will be incorporated into equation 2-6a. Following Aris, let us define the local velocity as

$$
\mathrm{U}(\mathrm{y}, \mathrm{z}) \equiv \overline{\mathrm{U}}[1+\mathrm{x}(\mathrm{y}, \mathrm{z})]
$$

where $x(y, z)$ is a function which describes the variation of velocity in the cross section, and the local eddy diffusivity as

$$
\epsilon_{x}=\epsilon_{y}=\epsilon_{z} \equiv D \psi(y, z)
$$

where $\mathrm{D}$ is the average value of the eddy diffusivity in the cross section and $\psi(y, z)$ is a function describing the distribution of eddy diffusivity. Isotropy of turbulence structure is implicitly assumed in equation 2-20. Using these definitions, equation 2-6a becomes

$\frac{\partial C}{\partial t}+\bar{U}(1+x) \frac{\partial C}{\partial x}=D \psi \frac{\partial^{2} C}{\partial x^{2}}+D \frac{\partial}{\partial y}\left(\psi \frac{\partial C}{\partial y}\right)+D \frac{\partial}{\partial z}\left(\psi \frac{\partial C}{\partial z}\right)$

Introducing the dimensionless parameters

$$
\left.\begin{array}{rl}
\xi & =\frac{x-\bar{U} t}{y_{n}} \\
\eta & =y / y_{n} \\
\zeta & =z / y_{n} \\
\tau & =D t / y_{n}^{2} \\
\mu & =\bar{U} y_{n} / D
\end{array}\right\}
$$

where $y_{n}$ is the depth of uniform flow in an open channel, equation 2-21 becomes

$\frac{\partial \mathrm{C}}{\partial \tau}+\mu \mathrm{X} \frac{\partial \mathrm{C}}{\partial \xi}=\psi \frac{\partial^{2} \mathrm{C}}{\partial \xi^{2}}+\frac{\partial}{\partial \eta}\left(\psi \frac{\partial \mathrm{C}}{\partial \eta}\right)+\frac{\partial}{\partial \xi}\left(\psi \frac{\partial \mathrm{C}}{\partial \xi}\right)$ 
Adapting the method derived by Taylor (1954a) for axi-symmetric turbulent pipe flow to twodimensional flow in an open channel, Elder (1959) simplified equation $2-23$ to

$$
\frac{\partial \mathrm{C}}{\partial \tau}+\mu \times \frac{\partial \mathrm{C}}{\partial \xi}=\frac{\partial}{\partial \eta}\left(\psi \frac{\partial \mathrm{C}}{\partial \eta}\right)
$$

by dropping the longitudinal turbulent diffusion term and restricting the analysis to vertical turbulent diffusion and longitudinal dispersion by convection. Following Taylor, Elder assumed that at large dispersion times $\partial C / \partial \tau \approx 0$, and that $C=C_{1}(\xi)+C_{2}(\eta)$ such that $\left|\partial C_{1} / \partial \xi\right|=$ constant. With these assumptions equation 2-24 simplifies further to

$$
\mu \times \frac{\partial C_{1}}{\partial \xi}=\frac{\partial}{\partial \eta}\left|\psi \frac{\partial C_{2}}{\partial \eta}\right| .
$$

After defining a convective dispersion coefficient, $K_{\text {, }}$, in terms of the flux of dispersant across a section at $\xi$,

$$
-\mathrm{K}_{\mathrm{C}} \frac{\partial \mathrm{C}_{1}}{\partial \xi} \equiv \int_{0}^{1} \mu \times \mathrm{C}_{2} \mathrm{~d}_{\eta}
$$

and substituting this definition into equation 2-25, Elder integrated equation 2-25 twice and obtained

$$
\mathrm{K}_{\mathrm{C}}=-\int_{0}^{1} \mu \mathrm{x} \int_{0}^{\eta} \frac{1}{\psi} \int_{0}^{\eta} \mu \mathrm{X} \mathrm{d} \eta \mathrm{d} \eta \mathrm{d} \eta .
$$

Following Taylor's (1954a) assumption that the convective dispersion and longitudinal turbulent diffusion coefficients are additive and that the latter is given by

$$
\mathrm{K}_{\mathrm{T}}=\int_{0}^{1} \psi(\eta) \mathrm{d} \eta=\frac{1}{\mathrm{D}} \int_{0}^{1} \epsilon \mathrm{d} \eta=1,
$$

the total longitudinal dispersion coefficient in dimensionless units is

$$
\mathrm{K}_{\xi}=\mathrm{K}_{\mathrm{C}}+1,
$$

and in dimensional units

$$
\mathbf{K}_{\mathbf{x}}=\mathbf{D}\left(\mathrm{K}_{\mathbf{C}}+\mathbf{1}\right)
$$

The assumptions made in going from equation 2-24 to equation 2-25 are rather drastic; however, they seem to be approximately true at large dispersion times. Therefore, values of the longitudinal dispersion coefficient obtained with the use of equation 2-26 are considered applicable only in the limiting case of large dispersion times.

Employing the von Karman-Prandtl logarithmic velocity distribution function, which applies equally well to either smooth or rough boundary conditions,

$$
\frac{\mathrm{U}-\overline{\mathrm{U}}}{\mathrm{U}_{\tau}}=\frac{1}{\kappa}(\ln \eta+1)
$$

and the Reynold's analogy (see section II-6) which states the equivalence of mass and momentum transfer,

$\epsilon_{\mathrm{y}} \equiv \frac{-\overline{v^{\prime} c^{\prime}}}{\frac{d C}{d y}} \approx \epsilon_{m} \equiv \frac{-\overline{u^{\prime} v^{\prime}}}{\frac{d U}{d y}} \approx \frac{U_{\tau}^{2} y_{n}(1-\eta)}{\frac{d U}{d \eta}}$,

Elder, after integrating equation 2-26, and adding the component for turbulent diffusion, obtained

$$
K_{x}=\left[\frac{0.404}{\kappa^{3}}+\frac{\kappa}{6}\right] y_{n} U_{\tau} \text {. }
$$

In equations $2-28$ and $2-29$

$$
\mathrm{U}_{\tau}=\sqrt{\tau_{\mathrm{o}} / \rho}=\sqrt{\mathrm{gy}_{\mathrm{n}} \mathrm{S}}
$$

is the shear velocity, $\kappa$ is the von Karman turbulence coefficient, and $\mathrm{S}_{e}$ is the slope of the energy gradient.

Using a parabolic velocity distribution function,

$$
\frac{\mathrm{U}-\overline{\mathrm{U}}}{\mathrm{U}_{\tau}}=\frac{1}{\kappa}\left(-3 \eta^{2}+6 \eta-2\right) \text {, }
$$

and the eddy diffusivity

$$
\epsilon_{\mathrm{y}}=\mathrm{D}=\frac{\kappa}{6} \quad \mathrm{y}_{\mathrm{n}} \mathrm{U}_{\mathrm{T}}
$$

in a similar way, Sayre and Chang (1966) obtained

$$
\mathrm{K}_{\mathrm{x}}=\left[\frac{0.457}{\kappa^{3}}+\frac{\kappa}{6}\right] \mathrm{y}_{\mathrm{n}} \mathrm{U}_{\tau}
$$

Sayre and Chang also showed that equation 2-30 agrees quite closely with experimental data provided that the conditions of a logarithmic velocity distribution in the vertical and no lateral velocity gradient are approximately satsifed. Furthermore, they found good agreement to occur for

$$
\tau=\frac{\mathrm{Dt}}{\mathrm{y}_{\mathrm{n}}^{2}}=\frac{\kappa}{6} \frac{\mathrm{U}_{\mathrm{T}}}{\mathrm{y}_{\mathrm{n}}} \mathrm{t} \approx \frac{\kappa}{6} \frac{\mathrm{U}_{\tau}}{\overline{\mathrm{U}}} \frac{\mathrm{x}}{\mathrm{y}_{\mathrm{n}}} \gtrsim 0.3
$$

which indicates that the dispersion time required for the applicability of equation 2-30 is not really very large.

Values of $\mathrm{K}$ determined experimentally in both the field and the laboratory, Glover (1964), Yotsukura, Smoot and Cahal (1964), Godfrey and Frederick (1963), have often greatly exceeded the value given by equation 2-30. This can be satisfactorily explained by the presence of lateral velocity gradients. Fischer (1966) formulated equation 2-26 for an assumed two-dimensional flow model in which the velocity varies with lateral position only. Using this model with various lateral velocity-distribution 
patterns similar to those found in natural channels, he showed that in wide channels the value of $K_{x}$ given by equation 2-30 can easily be exceeded by two or more orders of magnitude.

Aris (1956) approached the longitudinal dispersion problem with more rigor. He began by establishing the initial and boundary conditions for equation 2-23 as (1) $\tau=0$ : The initial spatial distribution of dispersant, $C(\xi, \eta, \zeta, 0)$, is known and is contained in a finite length of channel. (2) $\psi \partial \mathrm{C} / \partial \mathrm{n}=0$ at all boundaries including the water surface, where $n$ is the normal to the boundary. This states that no diffusion across the boundaries is permitted, or in other words that the boundaries are reflecting barriers.

$$
\text { (3) } \frac{1}{A} \iint_{A} \int_{-\infty}^{\infty} C(\xi, \eta, \zeta, \tau) d \xi d \eta d \xi=\text { constant, }
$$

where $A$ is the cross-sectional area of the channel, This states that the total amount of dispersant in the system remains constant. Next, Aris defined the $p^{\prime}$ th moment of the distribution with respect to $\xi$ along the filament $\eta, \zeta$ as

$$
C_{p}(\eta, \zeta, \tau) \equiv \int_{-\infty}^{\infty} \xi^{p} C(\xi, \eta, \zeta, \tau) d \xi
$$

and the $p^{\prime}$ th moment of the distribution along the whole channel as

$$
m_{p}(\tau) \equiv \overline{C_{p}}=\frac{1}{A} \iint_{A} C_{p} d \eta d \zeta
$$

Multiplying equation $2-23$ by $\xi^{p}$ and integrating with respect to $\xi$ from $-\infty$ to $+\infty$, Aris obtained the equation

$$
\begin{aligned}
& \left.\frac{\partial \mathrm{C}_{\mathrm{p}}}{\partial \tau}=\frac{\partial}{\partial \eta} \mid \psi \frac{\partial \mathrm{C}_{\mathrm{p}}}{\partial \eta}\right)+\frac{\partial}{\partial \zeta}\left|\psi \frac{\partial \mathrm{C}_{\mathrm{p}}}{\partial \underline{\varphi}}\right|+\mathrm{p} \mu \times \mathrm{C}_{\mathrm{p}-1} \\
& +p(p-1) \psi C_{p-2}
\end{aligned}
$$

for which the initial and boundary conditions become:

(1) $\tau=0$ : The initial distribution of moments over the cross section, $\mathrm{C}_{\mathrm{p}}(\eta, \zeta, 0)$, is fixed,

(2) $\psi \partial \mathrm{C}_{\mathrm{p}} / \mathrm{\partial n}_{\mathrm{n}}=0$ at the boundaries,

(3) $\frac{1}{A} \iint_{A} C_{0}(\eta, \zeta, \tau) d \eta d \zeta=$ constant.

Averaging equation 2-36 over the cross section, and making use of the divergence theorem and condition 2, Aris obtained

$$
\frac{d m p}{d \tau}=p \mu \overline{\frac{X C_{p}-1}{p}}+p(p-1) \overline{\psi C_{p}-2}
$$

where the bars denote averaging over the cross section as in equation 2-35. The conditions now become:

$$
\begin{aligned}
& \text { (1) } \tau=0: m_{p}(0) \text { is fixed, } \\
& \text { (3) } m_{0}(\tau)=\text { constant. }
\end{aligned}
$$

Although equations 2-36 and 2-37 cannot be solved for concentration distributions per se, solutions for the various moments are potentially capable of describing the dispersion process in considerable detail. A particularly attractive feature of the Aris moment equations is the considerable simplification achieved by the elimination of the variable $\xi$ from equation 2-23.

Aris did not solve the moment equations for any cases of turbulent flow in open channels. However, he did show that the moments, $m_{p}$, converge to the moments of the normal distribution as $\tau$ becomes large. This lends additional theoretical support to the applicability of the Fickian diffusion theory at large dispersion times.

Using Aris' analysis, Fischer (1964) showed for the case of a rectangular open channel with twodimensional flow that

$$
\mathrm{K}_{\xi}=\frac{1}{2} \frac{\mathrm{d} \sigma_{\xi}^{2}}{\mathrm{~d} \tau}=\frac{1}{2} \frac{\mathrm{d}}{\mathrm{d} \tau}\left[\frac{\mathrm{m}_{2}}{\mathrm{~m}_{0}}-\left(\frac{\mathrm{m}_{1}}{\mathrm{~m}_{0}}\right)^{2}\right]
$$

converges exactly to the functions given by Elder in equations $2-26$ and $2-27$ as $\tau$ becomes large. This apparently vindicates the questionable assumptions made by Taylor and Elder. All in all, the Aris moment equations seem to provide an extremely powerful method for the theoretical investigation of longitudinal dispersion processes. Strangely, it has scarcely been exploited.

Using a somewhat different set of dimensionless parameters, Yotsukura and Fiering (1964, 1966) solved the equation

$$
\frac{\partial C}{\partial t}+U \frac{\partial C}{\partial x}=\frac{\partial}{\partial y}\left(\epsilon_{y} \frac{\partial C}{\partial y}\right)
$$

for two particular velocity distributions by numerical methods with the aid of a digital computer. The longitudinal concentration distribution curves generated in the solutions resembled experimental curves in their approach to a normal distribution with increasing dispersion time from a highly skewed distribution at short dispersion times. However, because of the particular nature of the velocity distributions, general quantitative conclusions concerning the value of $K_{x}$ and the rate of convergence to the Fickian solution cannot readily be drawn from the results.

For a computation scheme in which the depth of flow was divided into 10 increments, the stepwise numerical method of Yotsukura and Fiering required more than 1.5 hours of IBM 7090 computer time to reach $\tau=1$. 
5. Applications of Dispersion Theory in Suspended Sediment Transport

The basic differential equation for the dispersion of suspended sediment, when the concentration by volume is small, is

$$
\frac{\partial C}{\partial t}+U_{s_{i}} \frac{\partial C}{\partial x_{i}}=\frac{\partial}{\partial x_{i}}\left[\epsilon_{s_{i}} \frac{\partial C}{\partial x_{i}}\right]
$$

which is the same as equation 2-5a except that the subscript $s$ here indicates reference to sediment particles. This equation or close facsimiles thereof has been proposed as a theoretical basis for the investigation of suspended sediment transport by Dobbins (1944), Hunt (1954), and McLaughlin (1959), among others. Upon introduction of the simplifications permitted by the assumption of uniform flow in the $x$ direction together with the additional assumptions that $\partial U / \partial z=0$ and $\partial C / \partial z=0$, equation $2-39$ reduces to

$\frac{\partial C}{\partial t}+U_{s} \frac{\partial C}{\partial x}=\epsilon_{s} \frac{\partial^{2} C}{\partial x^{2}}+\frac{\partial}{\partial y}\left[\epsilon_{s} \frac{\partial C}{\partial y}\right]+V_{s} \frac{\partial C}{\partial y}$.

With the exception of the additional convection term $\mathrm{V}_{\mathrm{S}} \partial \mathrm{C} / \partial \mathrm{y}$, where $\mathrm{V}_{\mathrm{S}}$ is the time-averaged fall velocity of the sediment particles, equation 2-40 has the same form as the corresponding equation for a fluid dispersant. Additional assumptions which are usually made in regard to equation 2-40 are that all the sediment particles have the same fall velocity, that $V_{S}$ is the same in turbulent and quiescent fluid, and that $\mathrm{U}_{\mathrm{S}}=\mathrm{U}$ at a point. With respect to the last assumption, however, it should be noted that the velocity of sediment averaged over the depth of flow,

$$
\bar{U}_{s}=\frac{\int_{0}^{y_{n}} U C d y}{\int_{0}^{y_{n}} C d y},
$$

is not the same as $\bar{U}$.

Although equation 2-40 in its complete form has never been solved, certain special cases which permit drastic simplifications have been solved. For example, for the steady state equilibrium conditions where both $\partial \mathrm{C} / \partial \mathrm{t}$ and $\partial \mathrm{C} / \partial \mathrm{x}$ equal zero and the deposition and entrainment rates are equal, equation 2-40 reduces to the well-known equation

$$
\epsilon_{s_{y}} \frac{\partial C}{\partial y}+v_{s} C=0
$$

which for many years has served as the basis for computing the vertical distribution of suspended sediment in alluvial channels. For the stated conditions and the diffusivity distribution function

$$
\epsilon_{\mathbf{s}_{\mathrm{y}}}=\epsilon_{\mathrm{m}}=\kappa \mathrm{U}_{\tau} \mathrm{y}_{\mathrm{n}} \eta(1-\eta)
$$

obtained by solving equation 2-29 for the logarithmic velocity distribution case, a solution of equation 2-42 is

$$
\frac{C(\eta)}{\int_{0}^{1} \operatorname{Cd} \eta}=f(\eta)=\left[\frac{1-\eta}{\eta}\right]^{\beta} \frac{\sin \pi \beta}{\pi \beta},
$$

where $\beta=V_{S} / \kappa U_{T}$ and $|\beta|<1$. Equation 2-44 in a slightly different form, was first presented by Rouse (1937). Elder (1959) put it in the form of a probability density function as given here.

For the case of a turbulence tank in which $\partial \mathrm{C} / \partial \mathrm{x}=0$ and $\epsilon_{\mathrm{S}_{\mathrm{y}}}=$ constant, Dobbins (1944) obtained a formal solution to the equation

$$
\frac{\partial C}{\partial t}=\epsilon_{s} \frac{\partial^{2} C}{\partial y^{2}}+V_{s} \frac{\partial C}{\partial y}
$$

which agreed well with the results of experiments in which the transient response to the shift from one steady state to another was investigated. From Dobbins' solution of equation 2-45, Camp (1944, 1946) developed a theory for sedimentation in settling tanks with turbulent flow. The rate at which suspended silt particles settled to the bed of the channel in the flume experiments of Sayre and Chang (1966) agreed reasonably well with predictions based on Camp's theory.

Experience in comparing solutions of equations 2-42 and 2-45 with other experimental data, Task Committee on Preparation of Sedimentation Manual (1963), indicates that the broad aspects of the theory are basically sound. However, at least two of the detailed aspects remain open to question. The first of these concerns the relationship between $\boldsymbol{\epsilon}_{\mathbf{s}_{\mathbf{i}}}$ and $\epsilon_{i}$. Empirically there is fairly general agreement that for small sediment particles $(d<0.1 \mathrm{~mm})$ with fall velocities in the Stokes range $\left({\tilde{v_{s}}}^{d} / v \lesssim 1\right), \epsilon_{S_{i}}$ and $\epsilon_{i}$ are approximately equal. This agrees essentially with the results of a theoretical treatment based on the equation of motion of a particle in a homogeneous turbulence field of infinite extent, Tchen (1947), Hinze (1959). For the limiting case of very short dispersion times the result of this analysis is

$$
\frac{\epsilon_{s_{i}}}{\epsilon_{i}}=\frac{a L_{t_{i}}+b^{2}}{a L_{t_{i}}+1}
$$

where

$$
a=\frac{36 p_{f} \nu}{\left(2 f p_{s}+\rho_{f}\right) d^{2}}, \quad b=\frac{3 p_{f}}{2 p_{s}+p_{f}}
$$

and $L_{t_{i}}$ is the Lagrangian integral time scale of turbulence, $v$ is the kinematic viscosity of the fluid, $d$ is the particle diameter, and $\rho_{f}$ and $\rho_{S}$ are respectively the mass densities of the fluid and the particles. As the dispersion time increases $\epsilon_{s_{i}}$ always tends to approach $\epsilon_{i}$, hence equation $2-46$ represents the critical case. According to equation 2-46 $\epsilon_{\mathrm{s}_{\mathrm{i}}} / \epsilon_{\mathrm{i}}<1$ whenever $\rho_{\mathrm{S}}>\rho_{\mathrm{f}}$. This apparently contradicts the 
experimental results of Singamsetti (1966) for the radial dispersion of sediment particles in a submerged turbulent water jet which indicate that $\epsilon_{\mathbf{S}_{i}} / \epsilon_{i}$ always exceeds one and increases continuously with particle size up to at least $V_{s} d / v=50$. These results in turn seem to be contradicted by those of Vanoni (1946) obtained from an investigation of the vertical distribution of suspended sediment in open-channel flows. If it is assumed that $\epsilon_{\mathrm{y}}=\epsilon_{\mathrm{m}}$, where $\epsilon_{\mathrm{m}}$ is the eddy diffusivity for momentum defined in equation 2-29, Vanoni's results showed that $\epsilon_{\mathbf{S y}}$ can be either somewhat larger or somewhat smaller than $\epsilon_{\mathrm{y}}$. However, because of the differences between submerged jet turbulence and boundary layer turbulence, neither of which constitute a homogeneous turbulence field of infinite extent, the above contradictions may well be more apparent than real.

The other detailed aspect of the theory behind equation 2-40 that has not been satisfactorily resolved is the extent to which the time-averaged fall velocity of sediment particles in a turbulence field may differ from the fall velocity of the same particles in still water. Even less is known about this than is known about the relative magnitudes of $\epsilon_{S_{i}}$ and $\epsilon_{i}$. However, the fact that in most cases reasonable agreement between theory and experiment is obtained by assuming that $\epsilon_{S_{i}}=\epsilon_{i}$ and that $V_{S}$ is the same under turbulent and quiescent conditions indicates that these assumptions are at least valid as first-order approximations.

A very rough approximation of the longitudinal dispersion coefficient for suspended sediment, $\mathrm{K}_{\mathrm{C}_{S}}$, was obtained by Elder (1959) from equation 2-26 with

$$
x=\frac{U-\bar{U}_{s}}{\bar{U}}
$$

where $\bar{U}_{S}$ is defined by equation 2-41. Assuming a parabolic velocity distribution in which case $\epsilon_{\mathrm{m}}$ is a constant, and assuming further that $\epsilon_{s_{y}}=\epsilon_{y}=\epsilon_{m}$. Elder obtained

$\mathrm{K}_{\mathrm{C}_{\mathrm{S}}}(\beta)=\frac{\mathrm{U}_{\max } \mathrm{y}_{\mathrm{n}}}{7560 \epsilon_{\mathrm{y}}}\left[64+21 \beta-308 \beta^{2}-210 \beta^{3}-35 \beta^{4}\right]$,

where $\beta=\mathrm{V}_{\mathrm{s}} / \kappa \mathrm{U}_{\tau}$. In the derivation of equation 2-47 it was also assumed that no deposit of sediment on the bed occurs and that the dispersing particles are distributed vertically according to equation 2-44. According to equation $2-47$ the ratio $\mathrm{K}_{\mathrm{C}_{\mathrm{S}}}(\beta) / \mathrm{K}_{\mathrm{C}_{\mathrm{S}}}(0)$ increases to a weak maximum of 1.0055 at $\beta=0.033$ and decreases rapidly to zero near $\beta=0.5$. Equation 2-47 does not agree well with the experimental results of Sayre and Chang (1966) for the longitudinal dispersion of silt-size particles where $\mathrm{K}_{\mathrm{C}_{\mathrm{S}}}(\beta) / \mathrm{K}_{\mathrm{C}_{\mathrm{S}}}(0)$ values as large as 2 in the neighborhood of $\beta=0.1$ were found. Due to differences in the boundary conditions, however, a fair comparison cannot be made.

In summary, although dispersion theory has not yet been successfully applied to predicting the longitudinal dispersion of suspended sediment particles, successful applications of simplified versions of the dispersion equation in some other areas of sediment transport suggest that such an application is possible.

\section{Reynolds' Analogy for the Equivalence of Mass and Momentum Transfer}

The concept of an analogy between the processes of mass and momentum transfer in turbulent flow arises from the similarity between the roles of the turbulent mass transfer term $\partial \overline{c^{\prime} u_{j}^{!}} / \partial x_{j}$ in equation
$2-2$,

$$
\frac{\partial \bar{C}}{\partial t}+\bar{U}_{j} \frac{\partial \bar{C}}{\partial x_{j}}=-\frac{\partial}{\partial x_{j}} \overline{c^{\prime} u_{j}^{\prime}}+\epsilon_{M} \frac{\partial^{2} \bar{C}}{\partial x_{j} \partial x_{j}}
$$

and the turbulent shear stress term $\rho \partial \overline{u_{i}^{\prime} u_{j}^{\prime}} / \partial x_{j}$ in
the momentum equation for turbulent flow

$$
\rho \frac{\partial \bar{U}_{i}}{\partial t}+\rho \bar{U}_{j} \frac{\partial \bar{U}_{i}}{\partial x_{j}}=-\rho \frac{\partial}{\partial x_{j}} \overline{u_{i}^{\prime} u_{j}^{\prime}}+\rho v \frac{\partial^{2} \bar{U}_{i}}{\partial x_{j} \partial x_{j}}+\bar{F}_{i}-\frac{\partial \bar{P}}{\partial x_{i}} .
$$

In fact, with the exception of the body force and pressure gradient terms, $\overline{\mathrm{F}}_{\mathrm{i}}$ and $\partial \overline{\mathrm{P}} / \partial \mathrm{x}_{\mathrm{i}}$, in equation $2-48$, the form of the two equations is the same. The analogy is carried further by introducing the Boussinesq turbulent exchange coefficients, $\epsilon_{i}$ for mass and $\epsilon_{m}$ for momentum, in such a way that

$$
\overline{c^{\prime} u_{i}^{\prime}}=-\epsilon_{i} \frac{\partial \bar{C}}{\partial x_{i}} \text { and } \rho \bar{u}_{i}^{\prime} u_{j}^{\prime}=-\rho \epsilon_{m}\left[\frac{\partial \bar{U}_{i}}{\partial x_{j}}+\frac{\partial \bar{U}_{j}}{\partial x_{i}}\right]
$$

express respectively the fluxes of mass and momentum due to turbulence. If we confine our attention to the transfer of mass and momentum in the $y$ direction in a channel with steady, uniform two-dimensional flow, the total transfer rates are for mass,

$$
\text { Mass Flux }=\left(\epsilon_{y}+\epsilon_{M}\right) \frac{\partial C}{\partial y}
$$

and for momentum,

$$
\text { Momentum Flux }=\rho\left(\epsilon_{m}+v\right) \frac{\partial U}{\partial y} .
$$

According to Rohsenow and Choi (1961) and Hinze (1959), the analogy is complete and $\epsilon_{\mathrm{y}}=\epsilon_{\mathrm{m}}$ if (1) the mechanisms which control mass transfer and momentum transfer are actually identical, (2) $\epsilon_{\mathrm{M}} \ll \epsilon_{\mathrm{y}}$ and $\nu \ll<\epsilon_{\mathrm{m}}$, (3) the momentum transfer is unaffected by the mass transfer. The third condition is automatically satisfied if the fluid properties of the dispersant and the dispersion medium ara the same. It would also appear to be satisfied for low concentrations of suspended particles with fall velocities in the Stokes range. The second condition is also satisfied in the open-channel flows considered 
here. Although eddy diffusion theory suggests that the first condition is satisfied, present knowledge of the processes of mass and momentum transfer is insufficient for an analytical proof. The analogy must therefore be justified on empirical grounds.

The momentum eddy diffusivity can be related to the velocity gradient in the following manner. For a channel with two-dimensional uniform flow, equation 2-48 for the transport of momentum in the $x$ direction reduces to

$$
F_{x}-\rho \frac{d}{d y} \overline{u^{\prime} v^{\prime}}+\rho v \frac{d^{2} U}{d y^{2}}=0
$$

The body force is

$$
F_{x}=\rho g S_{e}
$$

where $g$ is the acceleration of gravity and $S_{e}$ is the slope of the energy gradient. The boundary conditions are

$$
\begin{gathered}
y=0: \quad \overline{u^{\prime} v^{\prime}}=0, \rho v \frac{d U}{d y}=\tau_{0} \\
y=y_{n}: \quad \overline{u^{\prime} v^{\prime}}=0
\end{gathered}
$$

where $\tau_{0}$ is the shear stress acting on the bed. Integrating equation 2-49 with respect to $\mathrm{y}$, and using the boundary conditions to evaluate the constant of integration, we obtain

$$
\tau_{x y}=\tau_{0}\left(1-\frac{y}{y_{n}}\right)=-\rho \overline{u^{\prime} v^{\prime}}+\rho v \frac{d U}{d y} .
$$

Equation 2-50 has been verified experimentally by Laufer (1951) and Reichardt (1938) for two-dimensional flow in a wind tunnel. Introducing the momentum eddy diffusivity

$$
\epsilon_{\mathrm{m}}=\frac{-\overline{u^{\prime} v^{\prime}}}{\frac{d U}{d y}}
$$

and noting that $U_{\tau}=\sqrt{\frac{\tau}{\rho}}$, we get for $\nu \ll<\epsilon_{m}$,

$$
\epsilon_{\mathrm{m}} \approx \frac{\left.\mathrm{U}_{\tau}{ }^{2} \mid 1-\frac{\mathrm{y}}{\mathrm{y}_{\mathrm{n}}}\right)}{\frac{\mathrm{dU}}{\mathrm{dy}}}=\frac{\mathrm{U}_{\tau}^{2} \mathrm{y}_{\mathrm{n}}(1-\eta)}{\frac{\mathrm{dU}}{\mathrm{d} \eta}}
$$

as stated in equation $2-29$.

Using the von Karman-Prandtl velocity distribution function

$$
\frac{\mathrm{U}-\overline{\mathrm{U}}}{\mathrm{U}_{\tau}}=\frac{1}{\kappa}(\ln \eta+1)
$$

as an illustrative example, we obtain

$$
\frac{\mathrm{dU}}{\mathrm{d} \eta}=\frac{\mathrm{U}_{\tau}}{\kappa \eta}
$$

and

$$
\epsilon_{\mathrm{m}}=\kappa \mathrm{y}_{\mathrm{n}} \mathrm{U}_{\tau} \eta(1-\eta)
$$

According to equation 2-52, $\epsilon_{m}$ is distributed parabolically with respect to $\eta$ with a maximum value of $0.25 \mathrm{Ky}_{\mathrm{n}} \mathrm{U}_{\tau}$ occurring at $\eta=0.5$.

Turning now to the mass eddy diffusivity, Al-Saffar (1964) and Kalinske and Pien (1944) have demonstrated that within the limits of experimental error, $\epsilon_{\mathrm{y}} \approx \epsilon_{\mathrm{m}}$ in turbulent open-channel flow. Due to the difficulty of determining $\epsilon_{y}$, however, the limits of experimental error are not small.

In the development which follows it is assumed that Reynolds' analogy is valid, and $\epsilon_{\mathrm{y}}$ and $\epsilon_{\mathrm{m}}$ are used interchangeably. 


\section{SOLUTIONS OF ARIS' MOMENT EQUATIONS}

Of the various analytical approaches to longitudinal dispersion in turbulent open-channel flow, the most promising and apparently the least explored, appears to be the Aris moment equation method. With no sacrafice of rigor Aris' method affords a substantial simplification by reducing the number of variables in the dispersion equation.

\section{Development of the Working Equations}

For the sake of generality, let us begin with equation 2-40, considering a dispersant which settles toward the bed. Also let the following analysis be restricted to two-dimensional flow in an open channel in which all $\partial / \partial \zeta$ terms equal zero. Using the set of transformations given in equation $2-22$, along with equations 2-19 and 2-20 and the additional transformation

$$
v_{s}=v_{s} y_{n} / D
$$

equation 2-40 becomes

$$
\frac{\partial \mathrm{C}}{\partial \tau}+\mu \mathrm{X} \frac{\partial \mathrm{C}}{\partial \xi}=\frac{\partial}{\partial \eta}\left(\psi \frac{\partial \mathrm{C}}{\partial \eta}+\nu_{\mathrm{S}} \mathrm{C}\right)+\dot{\psi} \frac{\partial^{2} \mathrm{C}}{\partial \xi^{2}}
$$

The concentration, $C(\xi, \eta, \tau)$, refers now only to that component of dispersant which is entrained in the flow. The remainder, that which has settled to the bed, is stored there. It is assumed here that $\psi$ and $\mathrm{X}$ are properties of the flow and do not depend on the properties of the dispersant. The initial condition, corresponding to an instantaneous uniformly-distributed plane source at the origin is

$$
\begin{array}{lll}
\tau=0: & C(\xi, \eta, 0)=0 & \text { for } \xi \neq 0 \\
& C(\xi, \eta, 0)=\infty & \text { at } \xi=0 \\
& \int_{-\infty}^{\infty} C(\xi, \eta, 0) d \xi=1
\end{array}
$$

The boundary conditions, due to the fall-velocity term, are more complex than those treated by Aris. For the present case they are

$$
\eta=1: \quad \psi \frac{\partial \mathrm{C}}{\partial \eta}+v_{s} \mathrm{C}=0
$$

which states that there can be no transport of dispersant across the water surface, and

$$
\eta=0: \psi \frac{\partial \mathrm{C}}{\partial \eta}+(1-\alpha) v_{s} \mathrm{C}+\gamma \mathrm{W}=0 .
$$

Equation 3-5 is a very general boundary condition which permits the bed to behave either as an absorbing or a reflecting barrier and also permits temporary storage of the dispersant (e.g. sediment particles) on the bed of the channel. In equation 3-5 $\alpha$ is a bed absorbency coefficient representing the probability that a particle of dispersant coming into contact with the bed is deposited, $\mathrm{W}=\mathrm{W}(\xi, \tau)$ represents the amount of dispersant stored per unit area of bed surface, and $Y$ is an entrainment-rate coefficient, defined in such a way that $\gamma \Delta \tau$ is the probability that a typical particle resting on the bed is entrained in the flow during a short time interval of duration $\Delta \tau$. Following this line of reasoning, $\gamma \mathrm{W}$ represents an average rate of entrainment. Analogy with the process of radioactive decay, Evans (1955), suggests that the entrainment process can be represented as a Poisson process provided that the bed is covered by no more than a single layer of particles so that the probability of entrainment is the same for all particles. Galvin (1965) has described the entrainment process for the case where the bed is covered by more than a single layer.

If the dispersant is neutrally buoyant $\left(v_{S}=0\right)$ and there is no storage on the bed $(W=0)$, equation 3-2 reduces to the two-dimensional form of the equation treated by Aris with the simpler boundary condition $\psi \partial \mathrm{C} / \partial \eta=0$ at $\eta=0$ and $\eta=1$.

In conjunction with the boundary condition stated in equation 3-5, an equation for the deposition distribution function $W(\xi, \tau)$ is required. An equation which satisfies the requirement is

$$
\frac{\partial W}{\partial \tau}-\mu \frac{\partial W}{\partial \xi}=\alpha v_{S} C(\xi, 0+, \tau)-\gamma W .
$$

Like equation 3-2, equation 3-6 is a statement of the conservation of mass. The main difference is that the diffusion terms in equation $3-2$ are replaced by the exchange terms on the right side of equation 3-6. The initial condition for equation $3-6$ is $W(\xi, 0)=0$.

Employing the Aris moment transformations

$$
\mathbf{C}_{p} \equiv \int_{-\infty}^{\infty} \xi^{p} \mathrm{~d} d \xi \quad \text { and } \quad w_{p} \equiv \int_{-\infty}^{\infty} \xi^{p W d \xi} \text {, }
$$

equation 3-2 for the entrained component becomes

$$
\frac{\partial \mathrm{C}_{\mathrm{p}}}{\partial \tau}=\frac{\partial}{\partial \eta}\left|\psi \frac{\partial \mathrm{C}_{\mathrm{p}}}{\partial \eta}+v_{\mathrm{s}} \mathrm{C}_{\mathrm{p}}\right|+\mathrm{p} \mu \times \mathrm{C}_{\mathrm{p}-1}+\mathrm{p}(\mathrm{p}-1) \psi \mathrm{C}_{\mathrm{p}-2}
$$


with the initial condition

$$
\left.\begin{array}{lll}
\tau=0: & C_{p}(\eta, 0)=1 & \text { for } p=0 \\
& C_{p}(\eta, 0)=0 & \text { for } p>0
\end{array}\right\}
$$

and the boundary conditions

$$
\begin{aligned}
& \eta=1: \quad \frac{\partial C_{p}}{\partial \eta}+v_{s} C_{p}=0 \\
& \eta=0: \quad \psi \frac{\partial C_{p}}{\partial \eta}+(1-\alpha) v_{s} C_{p}+\gamma W_{p}=0 .
\end{aligned}
$$

The equation for the moments of the longitudinal deposition distribution is

$$
\frac{d W_{p}}{d \tau}=\alpha v_{s} C_{p}(0+, \tau)-p \mu W_{p-1}-\gamma W_{p},
$$

with the initial condition that $w_{p}(0)=0$.

For the special case of a neutrally buoyant dispersant where the boundary condition is $\psi \partial \mathrm{C}_{\mathrm{p}} / \partial \eta=0$ at $\eta=0$ and $\eta=1$, Aris' second moment transformation

$$
m_{p} \equiv \int_{0}^{1} C_{p} d \eta
$$

permits the further simplification of equation 3-7 to

$\frac{d m p}{d \tau}=p \int_{0}^{1} \mu \times C_{p-1} d \eta+p(p-1) \int_{0}^{1} \psi C_{p-2} d \eta .(3-12)$

A comparable simplification is not achieved in cases with the more complex boundary conditions stated in equations 3-9 and 3-10.

Even with the simplifications achieved by the Aris moment transformations, equations 3-7 and 3-12 are not amenable to purely analytical solutions except under very severe restrictions. A few analytical solutions have been obtained for the case of a constant eddy diffusivity $(\psi=1)$, and the limiting case of large dispersion times $(\tau \rightarrow \infty)$. In general, when $\psi$ varies with $\eta$, equation $3-7$, after separation of variables, leads to a set of non-homogeneous ordinary differential equations with variable coefficients. No standard method is available for solving this class of equations.

\section{Analytical Solutions for Simple Cases}

For easy reference, the cases for which analytical solutions of Aris' equations are available are listed in Table 3-1. Following is a brief description of each of these solutions

Case 1 - A simple case for which the writer has obtained an analytical solution to equations 3-7 and 3-12 is for a dispersant with $v_{s}=6 \beta=0$, e.g. a dye, in a flow with a parabolic velocity distribution

$$
\mu x=\frac{6}{\kappa^{2}}\left(-3 \eta^{2}+6 \eta-2\right)
$$

and the corresponding constant eddy diffusivity

$$
\epsilon=\mathrm{D}=\frac{\kappa}{6} \mathrm{y}_{\mathrm{n}} \mathrm{U}_{\mathrm{T}} \longrightarrow \psi=\frac{\epsilon}{\mathrm{D}}=1 \text {. }
$$

Considering first the equation for $C_{0}(n, \tau)(p=0$ in equation $3-7$ ), we get

\begin{tabular}{|c|c|c|c|c|c|c|}
\hline Case & $\begin{array}{l}\text { Variables for Which } \\
\text { Solution Obtained }\end{array}$ & $\begin{array}{l}\text { Velocity } \\
\text { Distribution }\end{array}$ & $\beta$ & $\alpha$ & $\gamma$ & Range of $T$ \\
\hline 1 & $C_{1}(\eta, \tau), K_{\xi}(\tau)$ & parabolic & 0 & & & all \\
\hline 2 & $C_{1}(n, \infty), \quad K_{\xi}(\infty)$ & logarithmic & 0 & & & $\tau \longrightarrow \infty$ \\
\hline 3 & $\mathrm{C}_{0}(\eta, \tau)$ & parabolic & all & 1 & $\mathbf{0}$ & all \\
\hline
\end{tabular}

$$
\frac{\partial \mathrm{C}_{0}}{\partial \tau}=\frac{\partial^{2} \mathrm{C}_{0}}{\partial \eta^{2}} \text {. }
$$

The solution for the appropriate initial and boundary conditions, obtained by inspection, is $\mathrm{C}_{0}=1$. This result merely states that

$$
C_{0}(\eta, \tau)=\int_{-\infty}^{\infty} C(\xi, \eta, \tau) d \xi .
$$

which is initially uniformly distributed with respect to $\eta$, remains uniformly distributed.

TABLE 3-1. KEY TO ANALYTICAL SOLUTIONS OF ARIS EQUATIONS 
The equation for the first moment

$$
C_{1}(\eta, \tau)=\int_{-\infty}^{\infty} \xi C(\xi, \eta, \tau) d \xi
$$

which is the mean displacement from $\xi=0$ of the dispersant contained in the plane $\eta$ at time $\tau$ is

$$
\frac{\partial C_{1}}{\partial \tau}=\frac{\partial^{2} C_{1}}{\partial \eta^{2}}+\frac{6}{\kappa^{2}}\left(-3 \eta^{2}+6 \eta-2\right) \text {. }
$$

This equation was solved by the standard method for linear non-homogeneous partial differential equations using Churchill's (1941) solution for the homogeneous part, to obtain

$\mathrm{C}_{1}(\eta, \tau)=\frac{6}{\kappa^{2}}\left[\left(\frac{\eta^{4}}{4}-\eta^{3}+\eta^{2}-\frac{2}{15}\right)+12 \sum_{n=1}^{\infty} \mathrm{e}^{-n^{2} \pi^{2} \tau} \frac{\cos n \pi \eta}{\mathrm{n}^{4} \pi^{4}}\right]$

Substitution of equation 3-15 into equation 3-12 gives $\frac{d m_{2}}{d \tau}=\frac{72}{\kappa^{4}} \int_{0}^{1}\left(-3 \eta^{2}+6 \eta-1\right) C_{1}(\eta, \tau) d \eta+2(3-16)$ and ultimately

$$
\begin{aligned}
K_{\xi} & =\frac{1}{2} \frac{d}{d \tau}\left[m_{2}-m_{1}^{2}\right]=\frac{1}{2} \frac{d m_{2}}{d \tau}=\frac{6}{k^{4}}\left[\frac{16}{35}\right. \\
& \left.+72 \int_{0}^{1}\left(-3 \eta^{2}+6 \eta-2\right) \sum_{n=1}^{\infty} e^{-n^{2} \pi^{2} \tau} \frac{\cos n \pi \eta}{n^{4} \pi^{4}} d \eta\right]+1 .
\end{aligned}
$$

For the limiting condition when $\tau \rightarrow \infty$, the integral expression goes to zero and

$$
\mathrm{K}_{\mathrm{x}}(\infty)=D K_{\xi}(\infty)=\left[\frac{0.457}{\kappa^{3}}+\frac{\kappa}{6}\right] \mathrm{y}_{\mathrm{n}} \mathrm{U}_{\tau}
$$

which is identical to equation 2-33 obtained earlier from Elder's equation. The convective component of equation 3-17, i.e., the bracketed part, is plotted in Figure 3-3 from which it is seen that $K_{\xi}$ converges to an asymptotic value at about $\tau=0.5$. distribution

Case 2 - For the logarithmic velocity

$$
\mu x=\frac{6}{\kappa^{2}}(\ln \eta+1)
$$

with the corresponding parabolically distributed eddy diffusivity obtained by using equation 2-51,

$$
\psi=\frac{\epsilon_{y}}{D}=6 \eta(1-\eta) \text {. }
$$

and $v_{\mathrm{S}}=0$, Yotsukura (1966) obtained

$$
\begin{aligned}
\mathrm{C}_{1}(\eta, \infty) & =-\int_{0}^{\eta} \frac{1}{\psi} \int_{0}^{n} \mu \mathrm{x} \mathrm{d} \eta \mathrm{d} \eta+\int_{0}^{1} \int_{0}^{\eta} \frac{1}{\psi} \int_{0}^{n} \mu \mathrm{x} \mathrm{d} \eta \mathrm{d} \eta \mathrm{d} \eta \\
& =\frac{1}{\kappa^{2}}\left[\frac{\pi^{2}}{6}-1-\sum_{\mathrm{n}=1}^{\infty} \frac{(1-\eta)^{\mathrm{n}}}{\mathrm{n}^{2}}\right] .
\end{aligned}
$$

From equations $3-12$ and $3-21$, as shown by Fischer (1964), the asymptotic value of the longitudinal dispersion coefficient is

$$
\begin{aligned}
\mathrm{K}_{\xi}(\infty) & =\frac{1}{2} \frac{\mathrm{dm} 2}{\mathrm{~d} \tau}=\int_{0}^{1} \mu \times \mathrm{C}_{1} \mathrm{~d}_{\eta}+\int_{0}^{1} \psi \mathrm{d} \eta \\
& =-\int_{0}^{1} \mu \mathrm{x} \int_{0}^{\eta} \frac{1}{\psi} \int_{0}^{\eta} \mu \mathrm{xd} \eta \mathrm{d} \eta \mathrm{d} \eta+1
\end{aligned}
$$

which leads, as was shown earlier, directly to Elder's (1959) equation 2-30. That $K_{\xi}$ is shown here to be composed of a convective part and a diffusive part in the exact manner postulated by Taylor (1954a) in equation 2-27 is a tribute to Taylor's remarkable intuition.

Case 3 - The solution obtained by Dobbins (1944) of equation 2-45 for the transient vertical concentration distribution of suspended sediment in a stationary homogeneous turbulence field is by analogy also the solution of equation 3-7 for $p=0, \alpha=1$, $y=0$ and the velocity and eddy diffusivity distributions specified in case 1 . Dobbins' solution is

$C_{0}(\eta, \tau)=2 e^{\frac{v_{s}}{2}(1-\eta)}$

$\left\{\sum_{n=1}^{\infty} \frac{\theta_{n} e^{-\left(\theta_{n}^{2}+\frac{\nu_{s}^{2}}{4}\right) \tau} \sin \theta_{n}\left(\cos \theta_{n} \eta+\frac{\nu_{s}}{2 \theta_{n}} \sin \theta_{n} \eta\right)}{\left(\theta_{n}^{2}+\frac{\nu_{s}^{2}}{4}\right)+\nu_{s}}\right\}$

where $\theta_{n}, n=1,2,3 \ldots$ are the successive real positive roots of the transcendental formula

$$
\cot \theta=\frac{\theta^{2}-\frac{v_{s}^{2}}{4}}{v_{8} \theta}
$$

In view of the complexity of the solutions for these three simple cases, the prospect of obtaining 
purely analytical solutions to cases which have applications in natural streams is not encouraging.

\section{Finite Difference Equations}

In contrast to the analytical solutions, the prospect of obtaining numerical solutions to the Aris equations by means of finite difference techniques using a digital computer is much more encouraging. The initial conditions do not entail any sharp gradients, and the elimination of $\xi$ by the moment transformation reduces the grid from three dimensions to two thereby greatly reducing the number of computations and the computer storage requirements.

The variables employed in the finite difference equations are illustrated schematically for a 10 layer model in Figure 3-1. The notation used here, where practical, is consistent with the notation used in the computer programs, which are included in the appendix. The depth of flow is divided into $\mathbf{N}$ equal increments of thickness DY $=\Delta \eta$. The boundaries between increments are numbered from $I=1$ to $I=N+1$. The number of time intervals of duration $\mathrm{DT}=\Delta \tau$, counted from the beginning of the dispersion process, is indicated by $J$, starting with $J=1$ at $\tau=0$ so that $\tau=(J-1) \mathrm{DT}$. The average value of $C_{p}(\eta, \tau)$ in the increment between $I$ and $I+1$ at the end of $J-1$ successive time intervals is defined as $C_{p}(I, J)$. Similarly, the velocity, $6 U(I) / \kappa^{2} *$ and the longitudinal component of the eddy diffusivity, EA(I), are defined as the average values of $\mu \mathrm{X}$ and $\psi$ in the increment between $I$ and $I+1$ so that

$$
\frac{6}{\kappa^{2}} \mathrm{U}(\mathrm{I}) \equiv \frac{1}{\mathrm{DY}} \int_{(\mathrm{I}-1) \mathrm{DY}}^{(\mathrm{I}) \mathrm{DY}} \mu \mathrm{X} \mathrm{d} \eta
$$

and

$$
\operatorname{EA}(\mathrm{I}) \equiv \frac{1}{\mathrm{DY}} \int^{(\mathrm{I}) \mathrm{DY}} \psi \mathrm{d} \eta
$$

$(I-1) D Y$

In contrast, the vertical component of the eddy diffusivity, which governs the exchange of dispersant
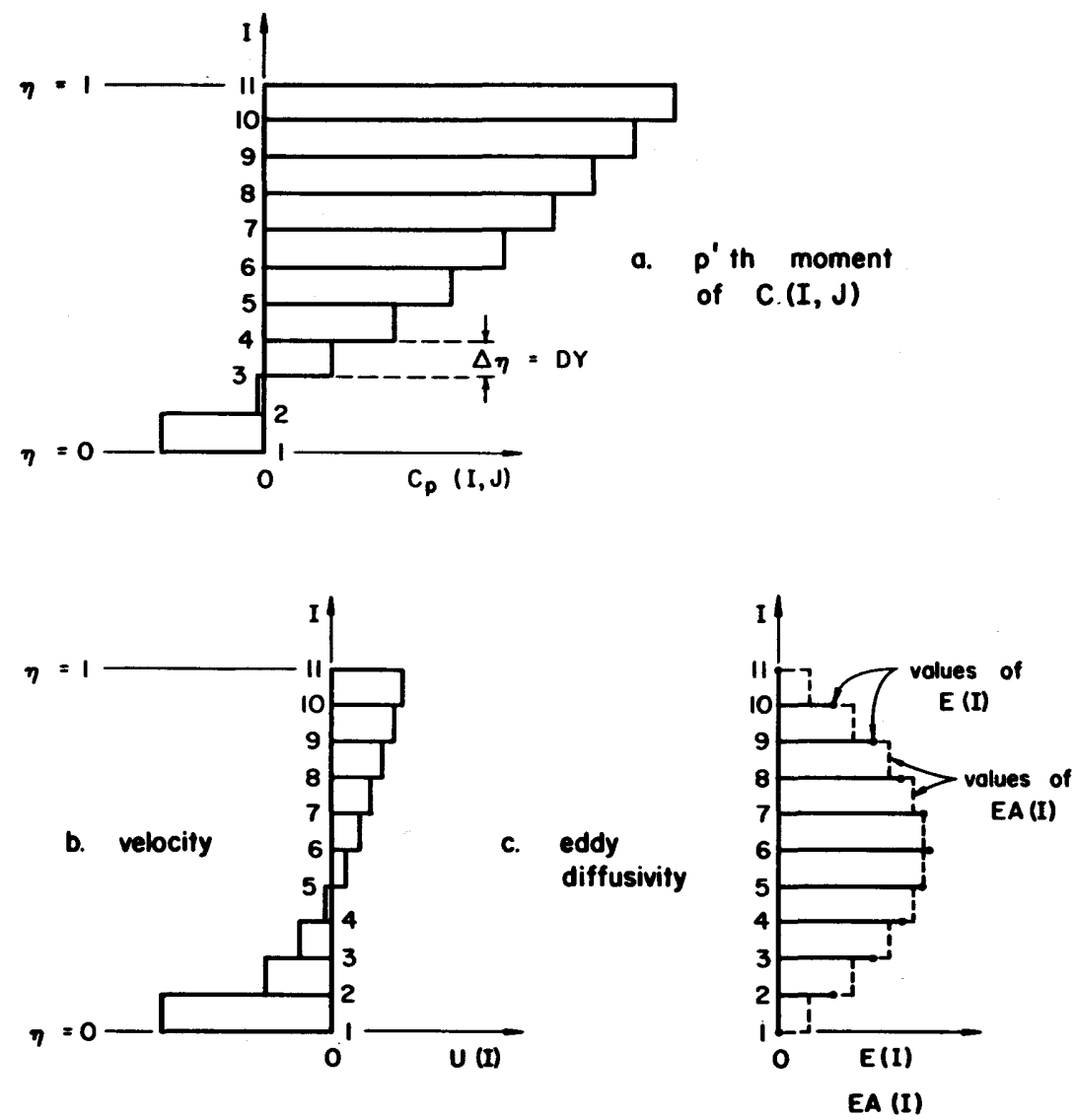

Figure 3-1. Definition sketch of variables in finite difference equations

\footnotetext{
* For velocity distributions other than logarithmic, $6 / \kappa^{2}$ is merely a proportionality constant and $\kappa$ is no longer the coefficient in the Prandtl momentum transfer equation $\mathrm{dU} / \mathrm{d} \eta=\mathrm{U}_{\tau} / \kappa \eta$.
} 
between adjacent increments, is defined as the value of $\psi$ at the boundary between the increments in question so that

$$
\mathrm{E}(\mathrm{I}) \equiv \psi(\eta), \text { where } \eta=(\mathrm{I}-1) \mathrm{DY}
$$

With these definitions, the finite difference approximations of the terms in equation 3-7 are

$$
\begin{aligned}
& \frac{\partial C_{p}}{\partial \tau} \rightarrow \frac{C_{p}(I, J+1)-C_{p}(I, J)}{D T}, \\
& \left.\frac{\partial}{\partial \eta} \mid \psi \frac{\partial C_{p}}{\partial \eta}+v_{s} C_{p}\right) \\
& \longrightarrow \frac{1}{D Y}\left\{\frac{E(I+1)}{D Y}\left[C_{p}(I+1, J)-C_{p}(I, J)\right]\right. \\
& \left.-\frac{E(I)}{D Y}\left[C_{p}(I, J)-C_{p}(I-1, J)\right]+v_{s}\left[C_{p}(I+1, J)-C_{p}(I, J)\right]\right\}, \\
& p \mu X C_{p-1}^{\longrightarrow} \frac{6}{\kappa^{2}} U(I) C_{p-1}(I, J),
\end{aligned}
$$

and

$p(p-1) \psi C_{p-2} \longrightarrow p(p-1) \operatorname{EA}(I) C_{p-2}(I, J)$

After some rearrangement of terms, the finite difference equation corresponding to equation 3-7 becomes for $1<\mathrm{I}<\mathrm{N}$

$$
\begin{aligned}
& C_{p}(I, J+1) \\
& =C_{p}(I, J)+\frac{D T}{D Y}\left\{\left[\frac{E(I+1)}{D Y}+v_{s}\right]\left[C_{p}(I+1, J)-C_{p}(I, J)\right]\right. \\
& \left.-\frac{E(I)}{D Y}\left[C_{p}(I, J)-C_{p}(I-1, J)\right]\right\}+p \frac{6 D T}{\kappa^{2}} U(I) C_{p-1}(I, J) \\
& +p(p-1) D T E A(I) C_{p-2}(I, J)
\end{aligned}
$$

The initial condition is

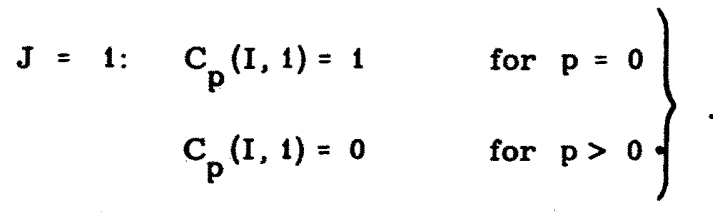

For $I=N$ and $I=1$ the boundary conditions given in equations 3-9 and 3-10 must be incorporated into the difference equation. Therefore, the substitutions

$\mathbf{I}=\mathbf{N}$ :

$\left[\frac{E(I+1)}{D Y}+\nu_{s}\right]\left[\dot{C}_{p}(I+1, J)-C_{p}(I, J)\right] \rightarrow-\nu_{s} C_{p}(I, J)$ and

$$
I=1 \text { : }
$$

$\frac{E(I)}{D Y}\left[C_{p}(I, J)-C_{p}(I-1, J)\right] \rightarrow-v_{s}(1-\alpha) C_{p}(I, J)-\gamma W_{p}(J)$

are made in equation.3-26. The difference equation corresponding to equation 3-11,

$$
\begin{aligned}
W_{p}(J+1) & =W_{p}(J)+D T \alpha \nu_{s} C_{p}(1, J)-p D T \mu W_{p-1}(J) \\
& -D T \gamma W_{p}(J)
\end{aligned}
$$

was obtained in a similar manner. The difference equation corresponding to equation $3-12$ is

$$
\begin{aligned}
\frac{M_{p}(J+1)-M_{p}(J)}{D T} & =p \frac{6}{\kappa^{2}} \sum_{I=1}^{N} U(I) C_{p-1}(I, J) D Y \\
& +p(p-1) \sum_{I=1}^{N} E(I) C_{p-2}(I, J) D Y .
\end{aligned}
$$

A critical step in the numerical solution of partial differential equations by finite difference methods is the selection of mesh size. If the numerical solution is to be stable and to converge satisfactorily to the true solution, certain conditions must be satisfied with respect to the way in which DT and DY approach zero. The subjects of stability criteria and rate of convergence are very complex and no conditions have ever been derived analytically which apply strictly to equation 3-26. According to Richtmyer (1957), the explicit difference equation corresponding to the one-dimensional Fickian diffusion equation,

$\frac{C(I, J+1)-C(I, J)}{\Delta t}=K \frac{C(I+1, J)-2 C(I, J)+C(I-1, J)}{(\Delta x)^{2}}$

is stable if

$$
\frac{\Delta t}{(\Delta x)^{2}} \leq \frac{1}{2 K}
$$


and the truncation error converges rapidly to zero if

$$
\frac{\Delta t}{(\Delta x)^{2}}=\frac{1}{6 K}
$$

Equations 3-26 and 3-32 are roughly comparable in that they are both explicit difference equations based on a diffusion equation, although equation 3-26 differs in that it includes additional lower order terms, and the diffusion coefficient $E(I)$ is variable. According to Richtmyer the presence of lower order terms does not appreciably affect stability. If it is assumed that the same conditions apply for equation 3-26, then

$$
\frac{\mathrm{DT}}{(\mathrm{DY})^{2}} \leq \frac{1}{2}
$$

for stability, and

$$
\frac{\mathrm{DT}}{(\mathrm{DY})^{2}} \approx \frac{1}{6}
$$

for vanishing truncation error.

In practice, these criteria were used as a guide in making the initial selection of mesh size. If the results indicated instability, DT/(DY) ${ }^{2}$ was reduced. Results which appeared to be satisfactory were checked for accuracy by repeating the computation for a smaller mesh size. The results of these trial and error mesh size investigations are summarized in Table 3-2. For most of the computer runs a mesh size of DT $=0.0005$ and $D Y=0.05$ were used. If the results appeared at all questionable the run was repeated for the reduced mesh size of DT = 0.00005 and $D Y=0.02$. Good agreement between these two mesh sizes was obtained in all cases.
4. Statistical Parameters for Describing Numerical Solutions

The immediate results of the iterative solutions of equations $3-26$ and 3-30 are difficult to interpret. To facilitate the description of results the computer programs were designed to calculate selected statistical parameters from the $\mathrm{C}_{\mathrm{p}}(\mathrm{I}, \mathrm{J})$ and $\mathrm{W}_{\mathrm{p}}(\mathrm{J})$ values. These parameters, which describe the principal statistical properties of the longitudinal concentration distribution curves, were calculated by means of the following formulas.

1. Convective component of overall longitudinal dispersion coefficient (only for cases where $v_{\mathrm{S}}=0$ ) from equation $3-31$

$$
\frac{D \kappa^{3}}{y_{n} U_{\tau}} K_{C}(\tau)=\kappa^{2} \sum_{I=1}^{N} U(I) C_{1}(I, J) D Y
$$

2. Area under longitudinal concentration distribution curve which can also be interpreted as the amount of dispersant remaining in suspension

a) for the I'th increment

$$
C_{0}(\eta, \tau)=C_{0}(I, J)
$$

\begin{tabular}{|c|c|c|c|c|}
\hline $\begin{array}{l}\text { Velocity and Eddy } \\
\text { Diffusivity Distri- } \\
\text { bution Functions }\end{array}$ & DT & DY & $\frac{\mathrm{DT}}{(\mathrm{DY})^{2}}$ & Comment on Results \\
\hline $\begin{array}{l}\mu \mathrm{x} \sim \text { Eq. } 3-13 \\
\quad \text { (parabolic) } \\
\psi=1\end{array}$ & $\begin{array}{l}0.005 \\
0.0005\end{array}$ & $\begin{array}{l}0.10 \\
0.05\end{array}$ & $\begin{array}{l}\frac{1}{2} \\
\frac{1}{5}\end{array}$ & $\begin{array}{l}\text { stable } \\
\text { stable, agreed closely } \\
\text { with preceding case }\end{array}$ \\
\hline $\begin{array}{l}\mu x \sim \text { Eq. } 3-19 \\
\text { (logarithmic) } \\
\psi=6 \eta(1-\eta)\end{array}$ & $\begin{array}{l}0.005 \\
0.001 \\
0.0005 \\
0.00005 \\
0.002\end{array}$ & $\begin{array}{l}0.10 \\
0.05 \\
0.05 \\
0.02 \\
0.10\end{array}$ & $\begin{array}{l}\frac{1}{2} \\
\frac{1}{2.5} \\
\frac{1}{5} \\
\frac{1}{8} \\
\frac{1}{5}\end{array}$ & $\begin{array}{l}\text { unstable } \\
\text { unstable } \\
\text { stable } \\
\text { stable, agreed closely } \\
\text { with preceding case } \\
\text { stable, not very good } \\
\text { agreement with two } \\
\text { preceding cases }\end{array}$ \\
\hline
\end{tabular}

b) for the entire flow field

$$
m_{0}(\tau)=\sum_{I=1}^{N} C_{0}(I, J) D Y
$$

TABLE 3-2. RESULTS OF TRIAL AND ERROR MESH-SIZE INVESTIGATIONS 
3. Instantaneous average velocity of all the dispersant in suspension

$$
\overline{\mu_{S}}(\tau)=\frac{1}{m_{0}(\tau)} \frac{6}{\kappa^{2}} \sum_{I=1}^{N} C_{0}(I, J) U(I) D Y .
$$
dispersant

4. Mean displacement from $\xi=0$ of entrained

a) for the $I^{\prime}$ th increment

$$
\bar{\xi}_{S}(\eta, r)=C_{1}(I, J) / C_{0}(I, J)
$$

b) for the entire flow field

$$
\bar{\xi}_{S}(\tau)=\frac{1}{m_{0}(\tau)} \sum_{I=1}^{N} C_{1}(I, J) D Y
$$

5. Variance for entrained dispersant

a) for the I'th increment

$$
\sigma_{S}^{2}(\eta, \tau)=\frac{\mathrm{C}_{2}(\mathrm{I}, \mathrm{J})}{\mathrm{C}_{0}(\mathrm{I}, \mathrm{J})}-\left[\bar{\xi}_{\mathrm{S}}(\eta, \tau)\right]^{2}
$$

b) for the entire flow field

$$
\sigma_{S}^{2}(\tau)=\frac{1}{m_{0}(\tau)} \sum_{I=1}^{N} C_{2}(I, J) D Y-\left[\bar{\xi}_{S}(\tau)\right]^{2}
$$

6. Skew coefficient for entrained dispersant

a) for the I'th increment

$S_{S}(\eta, \tau)=\frac{\frac{C_{3}(\mathrm{I}, J)}{C_{0}(\mathrm{I}, J)}-3 \bar{\xi}_{S}(\eta, \tau) \frac{C_{2}(\mathrm{I}, J)}{C_{0}(I, J)}+2\left[\bar{\xi}_{S}(\eta, \tau)\right]^{3}}{\left[\sigma_{S}^{2}(\eta, \tau)\right]^{3 / 2}}$

b) for the entire flow field

$$
\begin{aligned}
S_{S}(\tau) & =\frac{1}{\left[\sigma_{S}^{2}(\tau)\right]^{3 / 2}}\left\{\frac{1}{m_{0}(\tau)} \sum_{I=1}^{N} C_{3}(I, J) D Y\right. \\
. & \left.-\frac{3 \bar{\xi}_{S}(\tau)}{m_{0}(\tau)} \sum_{I=1}^{N} C_{2}(I, J) D Y+2\left[\bar{\xi}_{S}(\tau)\right]^{3}\right\} .
\end{aligned}
$$

The formulas used for calculating the parameters describing the longitudinal distribution of $W(\tau)$ are:

1. Area under distribution curve, which can also be interpreted as the amount of dispersant in storage on the bed

$$
\mathrm{W}_{0}(\tau)=\mathrm{W}_{0}(\mathrm{~J})
$$

2. Mean displacement from $\xi=0$ of deposited dispersant

$$
\bar{\xi}_{W}(\tau)=W_{1}(J) / W_{0}(J)
$$

3. Variance for deposited dispersant

$$
\sigma_{W}^{2}(\tau)=W_{2}(J) / W_{0}(J)-\left[\bar{\xi}_{W}(\tau)\right]^{2} .
$$

4. Skew coefficient for deposited dispersant

$$
S_{W^{\prime}}(\tau)=\frac{\frac{W_{3}(J)}{W_{0}(J)}-3 \bar{\xi}_{W^{(\tau)}} \frac{W_{2}(J)}{W_{0}(J)}+2\left[\bar{\xi}_{W^{\prime}}(\tau)\right]^{3}}{\left[\sigma_{W}^{2}(\tau)\right]^{3 / 2}} .
$$

The parameters describing the composite distribution of the entrained and deposited dispersant were calculated in a similar manner. The formulas are:

1. Total quantity of dispersant

$$
m_{0_{T}}(\tau)=m_{0}(\tau)+w_{0}(\tau) .
$$
dispersant

2. Instantaneous average velocity of all

$$
\bar{\mu}_{\mathrm{T}}(\tau)=\mathrm{m}_{0}(\tau) \bar{\mu}_{\mathrm{S}}(\tau)-\mathrm{W}_{0}(\tau) \mu
$$

3. Mean displacement from $\xi=0$ of all dispersant

$$
\bar{\xi}_{T}(\tau)=\frac{\sum_{I=1}^{N} C_{1}(I, J) D Y+W_{1}(J)}{m_{0}(\tau)} .
$$

4. Variance for all dispersant

$$
\sigma_{T}^{2}(\tau)=\frac{\sum_{I=1}^{N} C_{2}(I, J) D Y+W_{2}(J)}{m_{0}(\tau)}-\left[\bar{\xi}_{T}(\tau)\right]^{2} \cdot(3-52)
$$

5. Skew coefficient for all dispersant

$$
\begin{aligned}
& S_{T}(\tau)=\frac{1}{\left[\sigma_{T}^{2}(\tau)\right]^{3 / 2}}\left\{\frac { 1 } { m _ { 0 _ { T } } ( \tau ) } \left[\sum_{I=1}^{N} C_{3}(I, J) D Y+W_{3}(J)\right.\right. \\
& \left.\left.-3 \bar{\xi}_{T}(\tau)\left(\sum_{I=1}^{N} C_{2}(I, J) D Y+W_{2}(J)\right)\right]+2\left[\bar{\xi}_{T}(\tau)\right]^{3}\right\} \cdot(3-53
\end{aligned}
$$


5. Additional Background Information for Numerical Solutions

The two computer programs for solving equation. 3-26 and associated equations are given in the appendix. Both programs are written in Fortran II. Program I solves equation 3-26 for $p=1$ and $\nu_{\mathrm{S}}=6 \beta=0$, and computes $\mathrm{D}^{3} \mathrm{~K}_{\mathrm{C}}(\tau) / \mathrm{y}_{\mathrm{n}} \mathrm{U}_{\tau}$ as given in equation $3-35$, as the final result. The final version of Program II solves equations 3-26 and 3-30 for $p=0,1,2,3$, and computes some or all of the statistical parameters defined in equations 3-36 through 3-53 as the final result. In the earlier versions of Program II, only the dispersant suspended in the flow was considered. Program I served as a pilot program for the much more extensive Program II.

The input data required for Program I consisted of the velocity distribution $U(I)$, as defined in equation 3-23, and the eddy-diffusivity distribution $E(I)$. In addition to these, Program II required: EA(I), as defined in equation 3-24; the value of the von Karman turbulence coefficient, $T=\kappa$; the dispersant fall velocity term, $B=\beta=\nu_{S} / 6$; the bed adsorbency factor, $\mathrm{A}=\alpha$; and in cases involving the distribution of deposited dispersant, the probability of entrainment during unit time, $\mathbf{G}=\gamma$; and a term which is proportional to the mean velocity, $\mathrm{UA}=\kappa \mu / 6=\overline{\mathrm{U}} / \mathrm{U}_{\tau}$.

Velocity and eddy-diffusivity distributions for two different velocity distribution functions are shown in Figure 3-2. For the logarithmic velocity distribution function, the respective distributions are given by equations 3-19 and 3-20; and for the parabolic function, by equations 3-13 and 3-14. Mainly because of its widespread use, the logarithmic velocity distribu- tion function was used in nearly all of the computer runs. The parabolic velocity distribution function was used only in enough cases to establish that the numerical results given by Program I were in agreement with the analytical solution given in equation 3-17. The velocity and eddy diffusivity input information for Program II, for the case of the logarithmic velocity distribution with $D Y=0.05$, is tabulated in the appendix.

All of the cases for which numerical solutions were obtained are listed in Table 3-3. Program I was used for cases 1 and 2, and Program II for all the rest. The computers which were used and a comparison of the execution times required for the programs for different combinations of DY, DT, and the range of $\tau$ are listed in Table 3-4. The IBM 1620 facility is located in the Colorado State University Statistical Laboratory; the IBM 7094 at the Western Data Processing Center, University of California, Los Angeles; and the CDC 6600 at the National Center for Atmospheric Research, Boulder, Colorado.

The tremendous saving in computer time effected by employing the Aris moment transformations is illustrated by comparing the 1.5 hours on an IBM 7090 required for carrying out Yotsukura's and Fiering's (1964) numerical solution of equation 2-38 for $D Y=0.1$ to $\tau \simeq 1$, with the 1.3 minutes on the IBM 7094 required for carrying Program II for $D Y=0.05$ out to $\tau=3$. Thus, Program II, although it does not compute the actual concentration distributions, is very well suited for investigating the overall effects of various velocity distributions, eddy diffusivity distributions, boundary conditions and the like, on the longitudinal dispersion process.

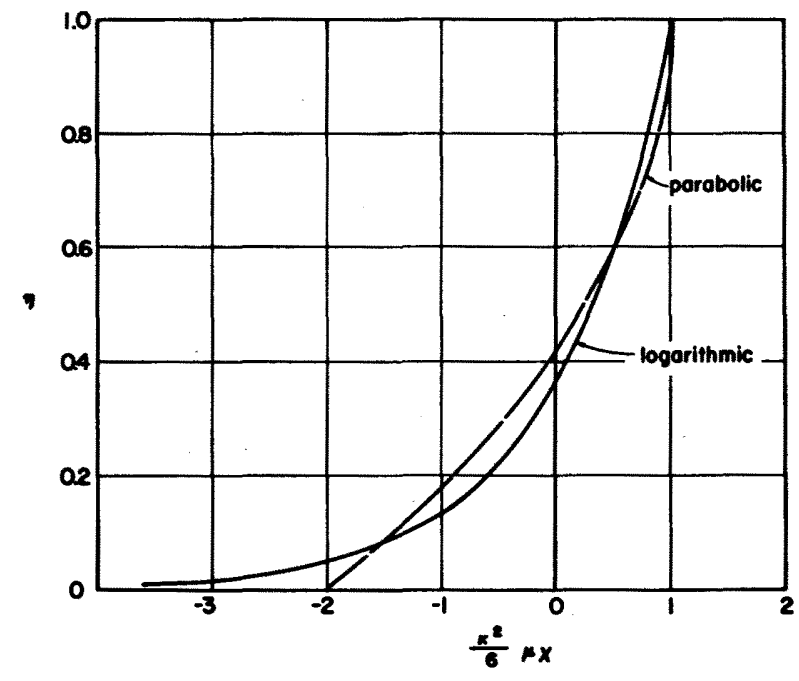

a. Velocity

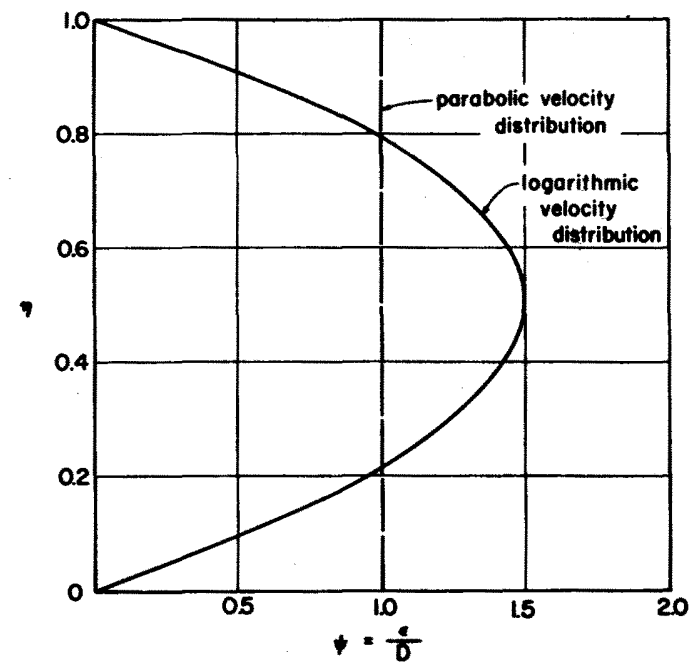

b. Eddy diffusivity

Figure 3-2. Distributions of velocity and eddy diffusivity with respect to depth 
TABLE 3-3. KEY TO NUMERICAL SOLUTIONS OF ARIS EQUATIONS

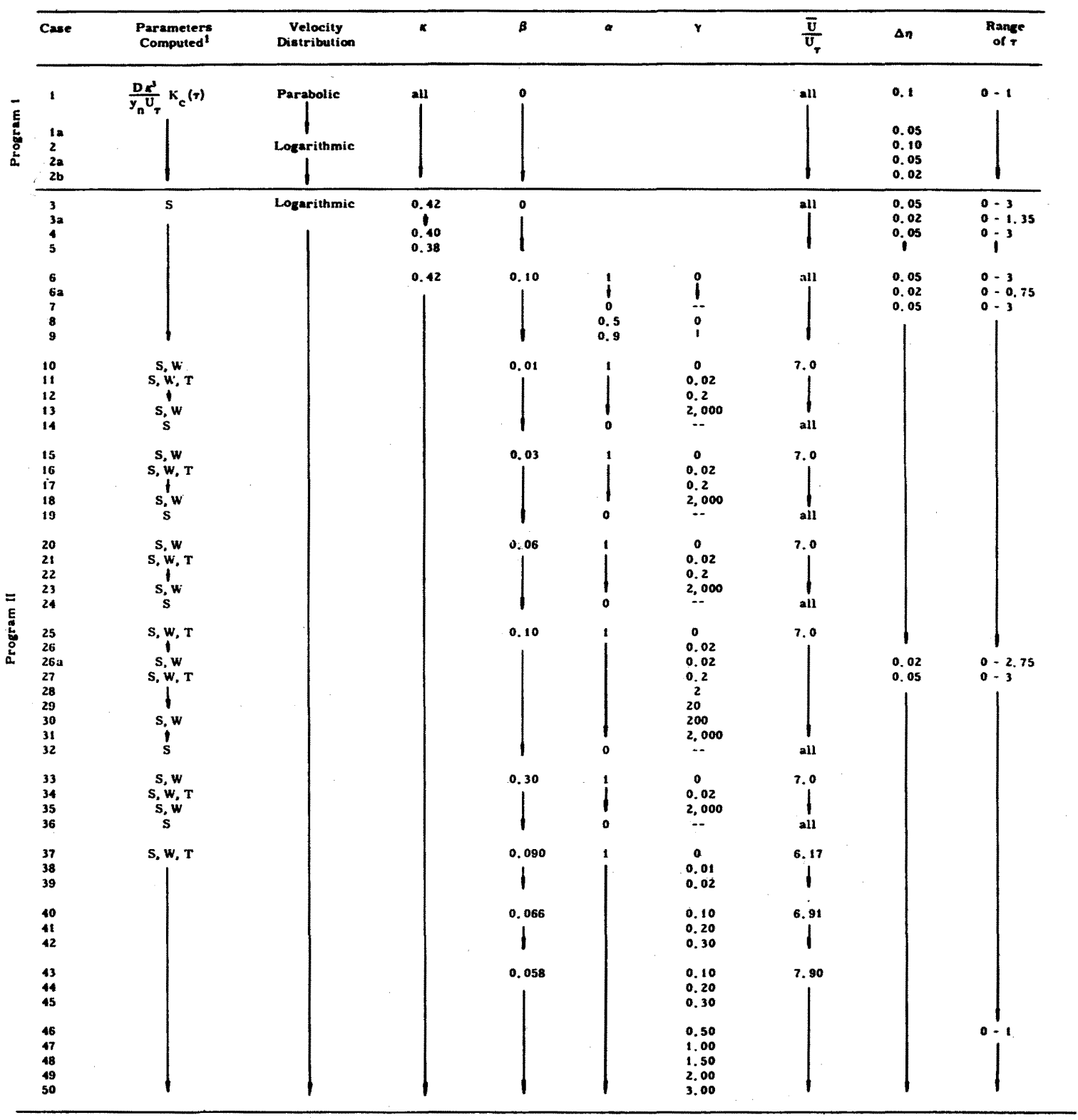

I $S$ denotes equations 3-36-3-44; $W$ equations 3-45-3-48; $T$ equations 3-49-3-53 
TABLE 3-4. APPROXIMATE COMPUTATION TIMES FOR NUMERICAL SOLUTIONS

\begin{tabular}{|c|c|c|c|c|c|}
\hline $\begin{array}{l}\text { Parameters } \\
\text { Computed }\end{array}$ & DY & DT & Computer & $\begin{array}{c}\text { Range } \\
\text { of } \tau\end{array}$ & $\begin{array}{c}\text { Computer } \\
\text { Execution } \\
\text { Time }\end{array}$ \\
\hline \multirow{2}{*}{$\frac{\mathrm{D} \kappa^{3}}{\mathrm{y}_{n} \mathrm{U}_{\tau}} \mathrm{K}_{\mathrm{C}^{(\tau)}}$} & 0.1 & 0.005 & IBM 1620 & $0-1$ & $20 \mathrm{~min}$. \\
\hline & 0.1 & 0.002 & IBM 1620 & $0-1$ & $32 \mathrm{~min}$. \\
\hline \multirow[t]{3}{*}{ (Program I) } & 0.05 & 0.0005 & IBM 1620 & $0-0.5$ & $90 \mathrm{~min}$. \\
\hline & 0.05 & 0.0005 & IBM 7094 & $0-1$ & $0.2 \mathrm{~min}$. \\
\hline & 0.02 & 0.00005 & IBM 7094 & $0-1$ & $2.2 \mathrm{~min}$. \\
\hline $\mathrm{S}, \mathrm{W}, \mathrm{T}$ & 0.05 & 0.0005 & IBM 7094 & $0-3$ & $1.3 \mathrm{~min}$. \\
\hline \multirow[t]{3}{*}{ (Program II) } & 0.05 & 0.0005 & CDC 6600 & $0-3$ & $13 \mathrm{sec}$. \\
\hline & 0.02 & 0.00005 & IBM 7094 & $0-0.75$ & $8.3 \mathrm{~min}$. \\
\hline & 0.02 & 0.00005 & CDC 6600 & $0-2.75$ & $6 \mathrm{~min}$. \\
\hline
\end{tabular}

\section{Results from Program 1}

The results of Program I for computing the convective component of the longitudinal dispersion coefficient are shown on Figure 3-3(a). In all cases, the coefficient increases rapidly at small values of the dimensionless dispersion time, $\tau$, and levels off to a constant value at about $\tau=0.5$.

A more detailed examination showed that in the parabolic velocity distribution case, the results for $\Delta \eta=0.1$ differ from the results for $\Delta \eta=0.05$ at most by a small amount in the third significant figure. Both numerical solutions agree very closely with the analytical solution given in equation 3-17. In the logarithmic velocity distribution case, the agreement between the numerical solutions for the three mesh sizes is not as good. The values of $\mathrm{D} \kappa^{3} \mathrm{~K}_{\mathrm{C}} / \mathrm{y}_{\mathrm{n}} \mathrm{U}_{\tau}$ at $\tau=1$ obtained in the numerical solution are compared in Table 3-5 with the asymptotic values for large dispersion times obtained by analytical methods. From these values and the curves it is concluded that adequate accuracy is obtained with the mesh size of $\Delta \eta=0.05, \Delta \tau=$ 0.0005 .

Comparison of the normalized dispersion coefficients in Figure 3-3(b) show that there is little

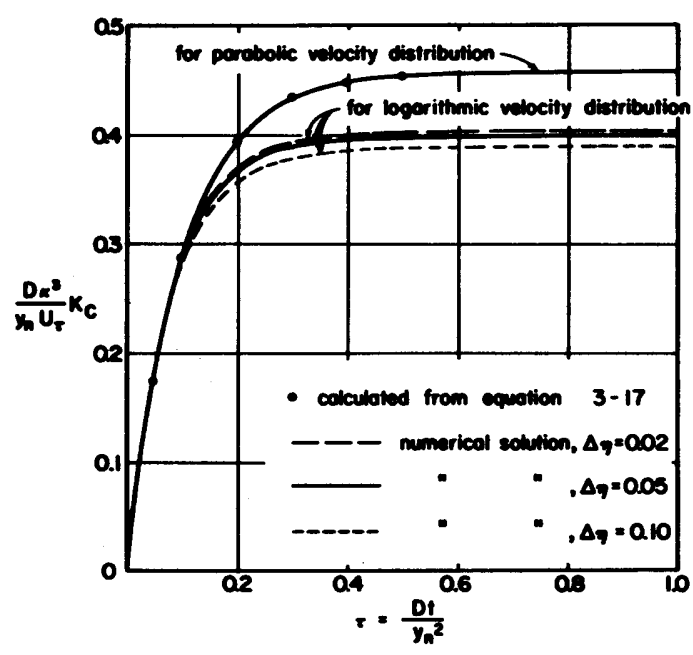

a. Non-normalized relationship

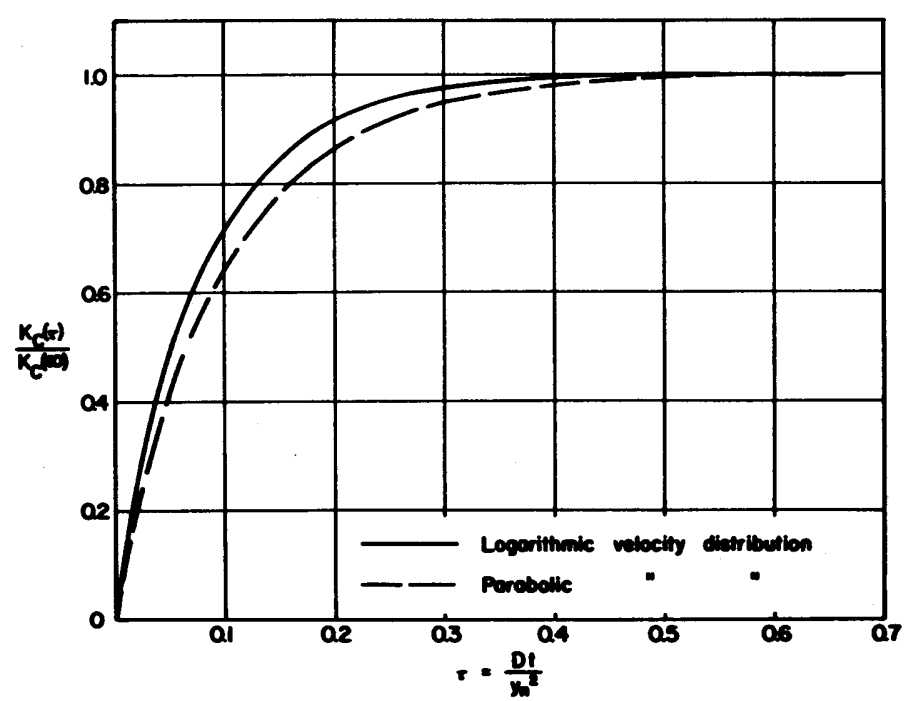

b. Normalized relationship

Figure 3-3. Convective component of longitudinal dispersion coefficient as a function of dispersion time 
TABLE 3-5. COMPARISON BETWEEN NUMERICAL AND ANALYTICAL RESULTS FOR CONVECTIVE COMPONENT OF LONGITUDINAL DISPERSION COEFFICIENT AT LARGE DISPERSION TIMES

\begin{tabular}{|cccc|}
\hline Velocity Distribution & $\Delta \eta$ & $\frac{\mathrm{D} \kappa^{3}}{\mathrm{y}_{\mathrm{n}} \mathrm{U}_{\tau}} \mathrm{K}_{\mathrm{C}}$ at $\tau=1$ & $\operatorname{Lim}_{\tau \rightarrow \infty} \frac{\mathrm{D} \kappa^{3}}{\mathrm{y}_{\mathrm{n}} \mathrm{U}_{\tau}} \mathrm{K}_{\mathrm{C}}$ \\
\hline Parabolic & $\begin{array}{c}\text { From Numerical } \\
\text { Solution }\end{array}$ & $\begin{array}{c}\text { From Analytical } \\
\text { Solution }\end{array}$ \\
\hline Logarithmic & 0.05 & 0.45711 & $\frac{16}{35}=0.45714$ \\
& 0.10 & 0.45706 & 11 \\
& 0.02 & 0.40294 & 0.4041 \\
& 0.05 & 0.39890 & $" 1$ \\
\hline
\end{tabular}

difference in the rate at which they approach the asymptotic limit. This suggests that the dispersion time required for $\mathrm{K}_{\mathrm{x}}$ to become approximately constant may not be very sensitive to the velocity distribution function.

\section{Results from Program II}

The results from Program II for each of the computer runs listed in Table 3-3 consisted of anywhere from about 500 to 3,000 items of information. It is obviously impractical to present all of the results. No more are presented than is necessary to illustrate the most important features of the longitudinal dispersion process as indicated by the solutions. The main objective is to show the influence of the various parameters and boundary conditions on the dispersion process and the presentation of results is organized with this end in mind.

a. Results for $\beta=0$ - The condition $\beta=0$, which indicates a dispersant with zero fall velocity, is applicable to the dispersion of solutions which have the same fluid properties as the dispersion medium, in this case water. The results for this case are presented first because they can be compared with previously established theories for large dispersion times, and also because they form a benchmark against which the results of the more complex cases can be compared.

In all runs with $\beta=0, \mathrm{C}_{0}(\eta, \tau)$ and $\mathrm{m}_{0}(\tau)$ remained equal to one for all $\tau$. This indicates that there is no net redistribution of dispersant in the vertical and that the condition of conservation of dispersant is satisfied. Also $\bar{\mu}_{S}(\tau)$ and $\bar{\xi}_{S}(\tau)$ remained equal to zero throughout all runs indicating that mean velocity and displacement of the dispersant are the same as for the flow. These results, which had of course been anticipated, gave some indication that the program was satisfactory.
In Figure 3-4, the variance $\sigma_{\xi}^{2}=\sigma_{S}^{2}(\tau)$, is shown as a function of $\tau$ for flows with a logarithmic velocity distribution but different values of $\kappa$. The general form of the relationship, in which the rate of change of the variance increases initially and later becomes constant, is consistent with that usually observed in experiments and also with the prediction of Taylor's theory of diffusion by continuous movements. The check run for $\kappa=0.42$ with $\Delta \eta=0.02$ agrees closely with the results of the corresponding run with $\Delta \eta=0.05$. The convention, introduced here, of representing the results for runs with $\Delta \eta=$ 0.05 by a solid curve and the results for runs with $\Delta \eta=0.02$ by small black circles is used throughout except when there are specific indications to the contrary.

As the overall longitudinal dispersion coefficient, $K_{\xi}$, is composed of a convective and a diffusive part, so also is the variance, $\sigma_{\xi}^{2}$. Combining equation 2-27 with the dimensionless counterpart of equation $2-13$,

$$
\mathrm{K}_{\xi}=\frac{1}{2} \frac{\mathrm{d} \sigma_{\xi}^{2}}{\mathrm{~d} \tau}
$$

and integrating, we get

$\sigma_{\xi}^{2}=2 \int_{0}^{\tau} \mathrm{K}_{\xi} \mathrm{d} \tau=2 \int_{0}^{\tau}\left(\mathrm{K}_{\mathrm{C}}+1\right) \mathrm{d} \tau=2 \int_{0}^{\tau} \mathrm{K}_{\mathrm{C}} \mathrm{d} \tau+2 \tau$.

Therefore, the convective component of the variance is

$$
\sigma_{C}^{2}=2 \int_{0}^{T} K_{C} d \tau=\sigma_{\xi}^{2}-2 \tau
$$




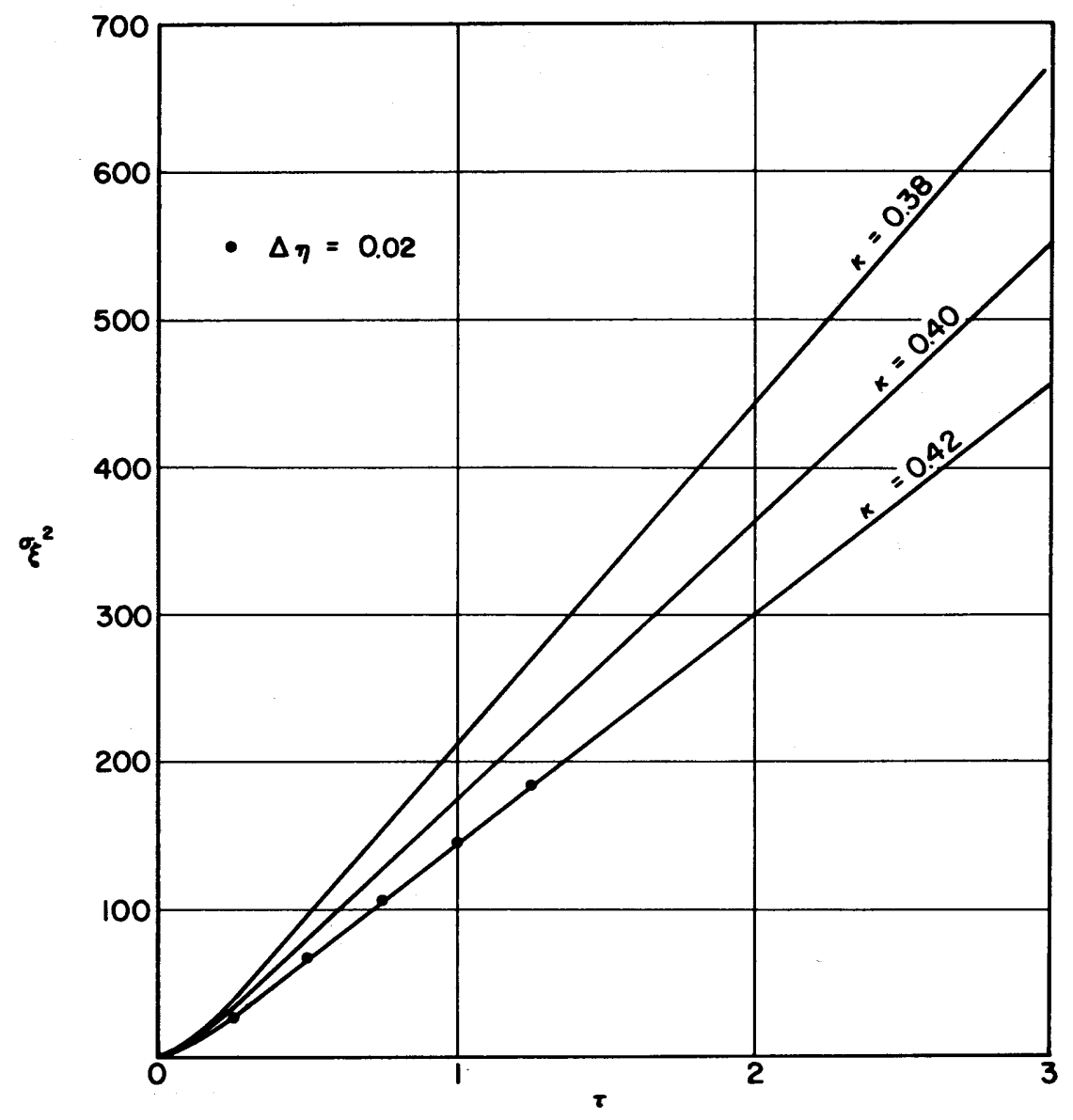

Figure 3-4. Variance of longitudinal concentration distribution as a function of dispersion time in flows with a logarithmic velocity distribution for $\kappa=0.42,0.40$ and 0.38

The results in Figure 3-4 can be generalized for all $\kappa$ by multiplying the convective component of the variance by $\mathrm{D} \kappa^{3} / \mathrm{y}_{\mathrm{n}} \mathrm{U}_{T}$ as shown in Figure 3-5. The corresponding relationship for the parabolic velocity distribution case, which is also shown in Figure 3-5, was obtained from the results of Program I by using equation 3-55. If the process is reversed by taking the derivative and dividing by two,

$$
\frac{\mathrm{D} \kappa^{3}}{\mathrm{y}_{\mathrm{n}} \mathrm{U}_{\tau}} \mathrm{K}_{\mathrm{C}}=\frac{1}{2} \frac{\mathrm{D} \kappa^{3}}{\mathrm{y}_{\mathrm{n}} \mathrm{U}_{\tau}}\left[\frac{\mathrm{d}}{\mathrm{d} \tau}\left(\sigma_{\xi}^{2}-2 \tau\right)\right]
$$

the functions in Figure 3-5 become identical to those in Figure 3-3(a). Therefore, the discussion of the results in Figure 3-3 apply here also, and it is demonstrated furthermore that the results of Programs I and II are in agreement.

The skew coefficient of the longitudinal concentration distribution, where $S(\tau)=S_{S}(\tau)$ as defined in equation 3-44, is shown as a function of $\tau$ in Figure 3-6 for a flow with the logarithmic velocity distribution. The results indicate that the concentration distributions are negatively skewed, and that they tend to become more symmetrical with increasing dispersion time. However, the rate of approach to symmetry, $S(\tau)=0$, is much slower than the rate at which $\mathrm{K}_{\xi}$ converges to an asymptotic value. The results for the check run with $\Delta \eta=0.02$ here also agree quite closely with the results for $\Delta \eta=0.05$, although not as closely as the results for the variance in Figure 3-4. Runs were also made for other $\kappa$ values. The results of these showed that the distributions tend to become slightly more skewed as $\kappa$ is decreased. However, the fact that a reduction of $\kappa$ from 0.42 to 0.38 caused only about a one percent change in the skew coefficient demonstrates that skewness is quite insensitive to changes in $\kappa$.

Figure 3-7 shows how the properties of the longitudinal concentration distribution along a streamline are distributed with respect to depth and how the distribution of these properties changes with increasing dispersion time. The displacement of the means, $\bar{\xi}(\eta, \tau)$, shown in Figure 3-7(a), tends to spread longitudinally with increasing dispersion time until $\tau=0.5$ when a state of equilibrium is reached. The equilibrium distribution agrees almost exactly with equation 3-21 which was obtained analytically. 


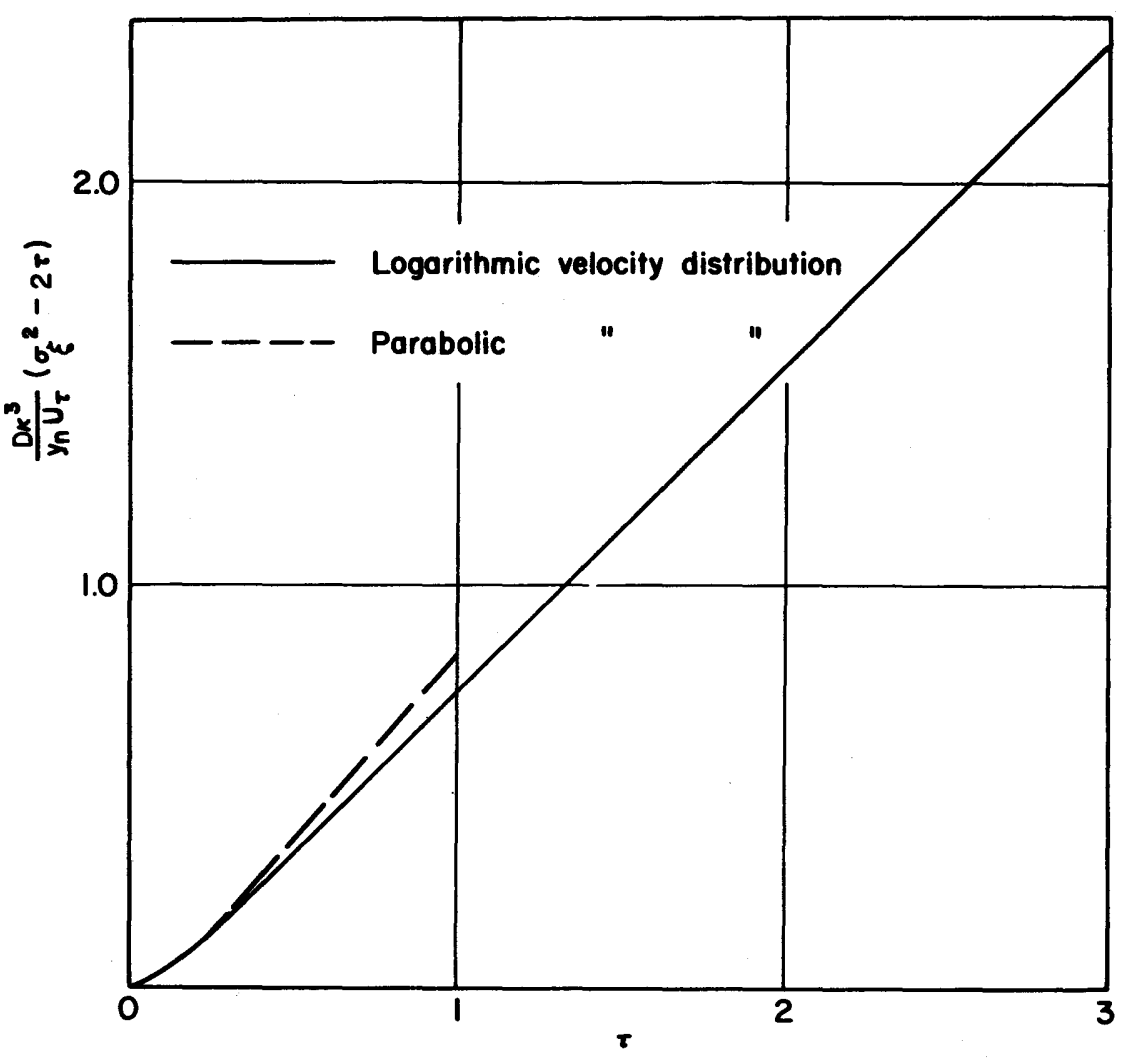

Figure 3-5. Convective component of variance of longitudinal concentration distribution as a function of dispersion time

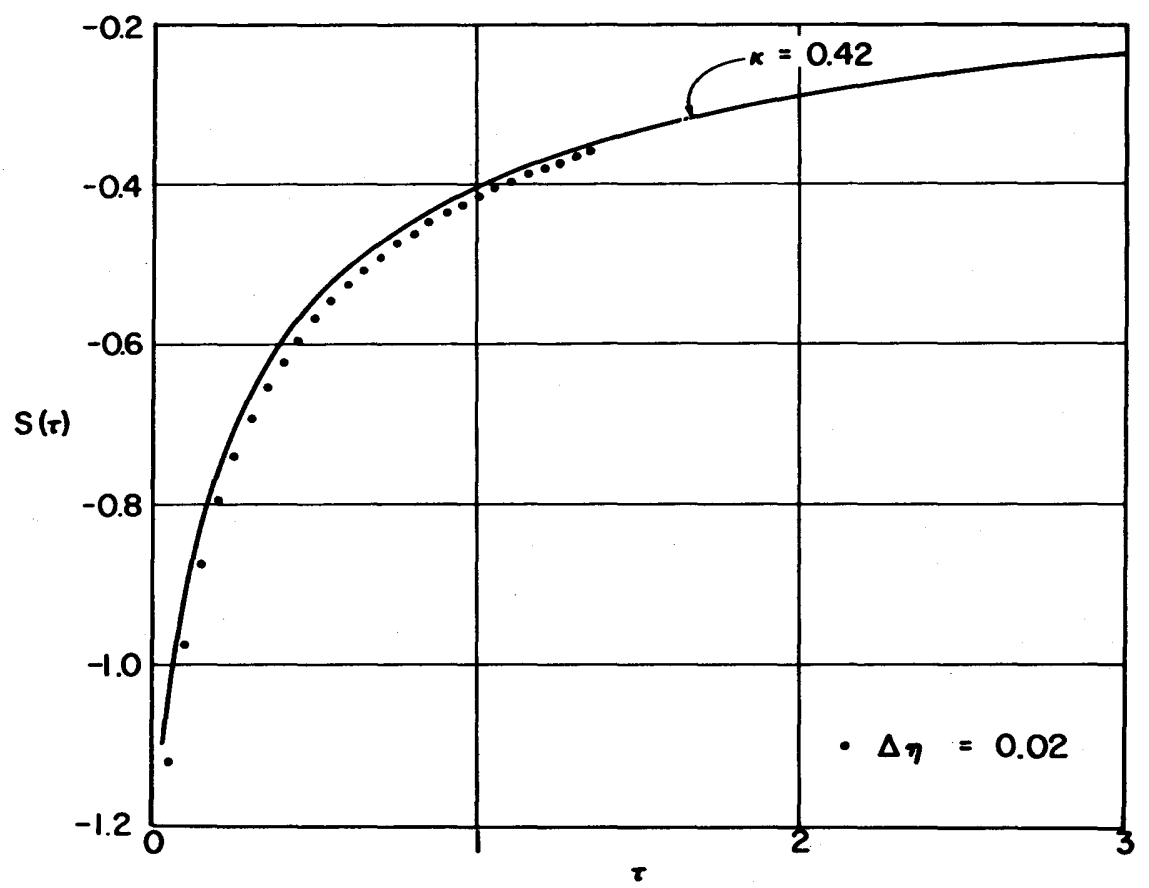

Figure 3-6. Skew coefficient of longitudinal concentration distribution as a function of dispersion time. 


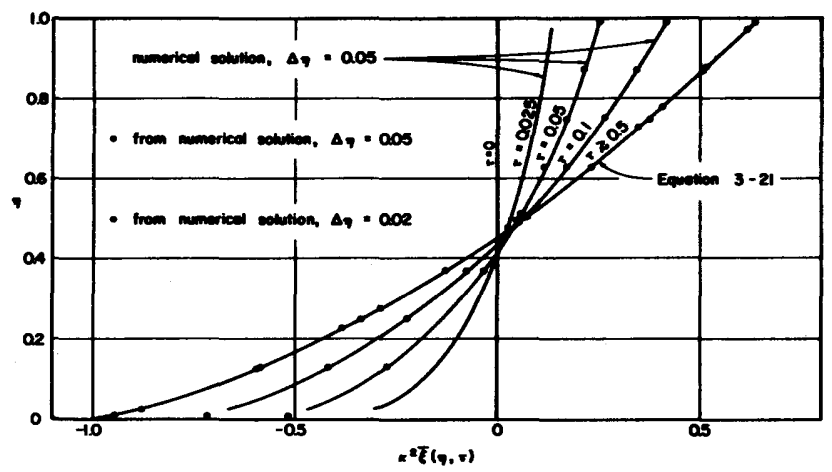

a. Mean displacement

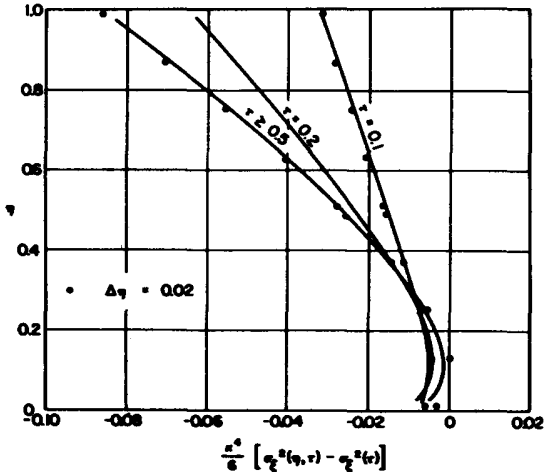

b. Variance

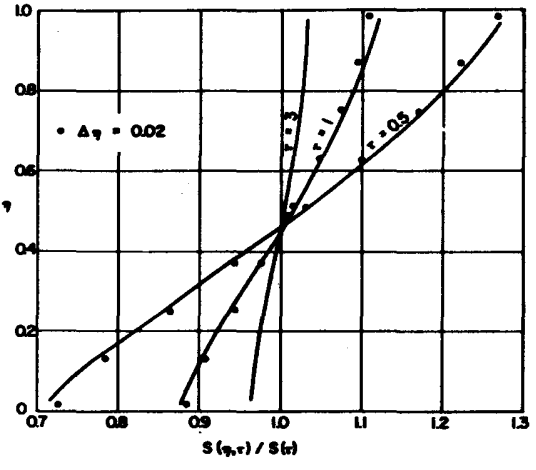

c. Skew coefficient

Figure 3-7. Distribution of properties of longitudinal concentration distribution along a streamline, with respect to depth in a flow with a logarithmic velocity distribution

The distribution of the difference between the variance along a streamline, $\sigma_{\xi}^{2}(\eta, \tau)$, and the overall variance, $\sigma_{\xi}^{2}(\tau)$, shown in Figure $3-7(b)$, also reaches a state of equilibrium at about $\tau=0.5$. Because the overall variance is greater than the variance along any streamline, the difference, $\sigma_{\xi}^{2}(\eta, \tau)-\sigma_{\xi}^{2}(\tau)$, is always negative. Keeping this in mind, it is seen from Figure $3-7(b)$ that $\sigma_{\xi}^{2}(\eta, \tau)$ decreases going from $\eta=0.12$ to $\eta=1$, and a maximum occurs near $\eta=0.12$.

The distribution of the skew coefficients, shown in Figure 3-7(c), unlike the distributions of the mean and variance, tends to become more uniform with increasing dispersion time. The skew coefficient for the distribution of dispersant along each streamline $S(\eta, \tau)$ approaches the overall skew coefficient which in turn is approaching zero.

The results shown on Figure 3-7 apply equally well for either smooth or rough boundary conditions. This is apparent when it is recalled that the velocity defect relationship given in equation $2-28$ is the same for both cases, Taking these results together, it is significant that the time required for stabilization of the mean displacement and variance distributions is the same as that required for the longitudinal dispersion coefficient to level off to a constant value.

Evidently a state of equilibrium between the convective and diffusive transfer rates is attained, which permits the overall longitudinal dispersion to behave as a one-dimensional process. As suggested earlier, when $\tau \geq 0.5$, the group of terms

$$
-\mu \chi \frac{\partial C}{\partial \xi}+\psi \frac{\partial^{2} \mathrm{C}}{\partial \xi^{2}}+\frac{\partial}{\partial \eta}\left(\psi \frac{\partial \mathrm{C}}{\partial \eta}\right)
$$

in equation 3-2 interact in such a way that their collective behavior can be represented by the single term $K_{\xi} \partial^{2} \bar{C} / \partial_{\xi}{ }^{2}$. These results support the intuitive arguments of Taylor (1954a) and Elder (1959) that equation 2-25 becomes a valid approximation of equation 2-24 at large dispersion times.

Parameters such as the mean displacement, variance and skew coefficient do not ordinarily convey a picture of the longitudinal concentration distribution. This is one drawback of the Aris method. However, with the aid of the Pearson Type III distribution function, which is essentially a three parameter gamma distribution, it is possible to construct distribution curves which have the same values of $\bar{\xi}, \sigma_{\xi}^{2}$ and $S$ as 
given by the numerical solution. The equation for the Pearson Type III distribution is

$$
f(\xi ; m, b, a)=\frac{1}{|b| \Gamma(a)}\left|\frac{\xi-m}{b}\right|^{a-1} e^{-\left(\frac{\xi-m}{b}\right)},
$$

and the mean, variance and skew coefficient corresponding thereto are

$$
\left.\begin{array}{l}
\bar{\xi}=m+b a \\
\sigma_{\xi}^{2}=b^{2} a \\
S=2 / \sqrt{a}
\end{array}\right\}
$$

The curves shown in Figure 3-4 and 3-6 can be closely approximated by the empirical formulas

$$
\left.\begin{array}{l}
\sigma_{\xi}^{2}=\frac{1}{\kappa^{4}}(4.85 \tau-0.40) \\
S=-0.420(\tau+0.10)^{-1 / 2} .
\end{array}\right\}
$$

Using the knowledge that $\bar{\xi}=0$, equations $3-57$ and 3-58 were solved simultaneously to obtain functional relationships between the Pearson parameters $a, b$ and $m$, and the dispersion time $\tau$. These relationships were then substituted into equation 3-56 to obtain estimates of the longitudinal concentration distribution, $f(\xi ; \tau)$. Concentration distribution curves, estimated in this manner, are shown as the solid curves in Figure 3-8. For comparison, the dashed curves which represent the Fickian equation

$$
f(\xi ; \tau)=\frac{1}{2 \sqrt{\pi K_{\xi} \tau}} e^{-\frac{\xi^{2}}{4 K_{\xi} \tau}}
$$

are also shown. It is apparent that the estimated concentration distributions tend to converge to the normal distribution as the dispersion time increases.

b. Interpretation of results for $\beta=0$ in terms of diffusion by continuous movements - The results of the numerical solutions for $\beta=0$, as evidenced by the relationship between $\sigma_{\xi}^{2}$ and $\tau$ and the apparent convergence of $f(\xi ; \tau)$ to the Fickian equation, appear to support the view expressed in Section II-3 that longitudinal dispersion in an axially-homogeneous, open-channel flow fits within the framework of Taylor's (1921) theory of diffusion by continuous movements. Restating the fundamental relationships of Taylor's theory using our present notation, we have

$$
\begin{aligned}
& \frac{1}{2} \frac{d \sigma_{\xi}^{2}(\tau)}{d \tau}=\frac{1}{2} \frac{d}{d \tau} \overline{\xi^{2}}=\overline{\xi \frac{d \xi}{d \tau}}=\overline{\xi u_{L}^{\prime}(\tau)} \\
& =u_{L}^{\prime}(\tau) \int_{0}^{\tau} u_{L}^{\prime}\left(\tau_{1}\right) d \tau_{1}=\int_{0}^{\tau} \overline{u_{L}^{\dagger}(\tau) u_{L}^{\prime}\left(\tau_{1}\right) d \tau_{1}},
\end{aligned}
$$

where $u_{L}^{\prime}(\tau)$ is the Lagrangian velocity in the $\xi, \eta, \tau$ system. As before, the overbars denote an ensemble
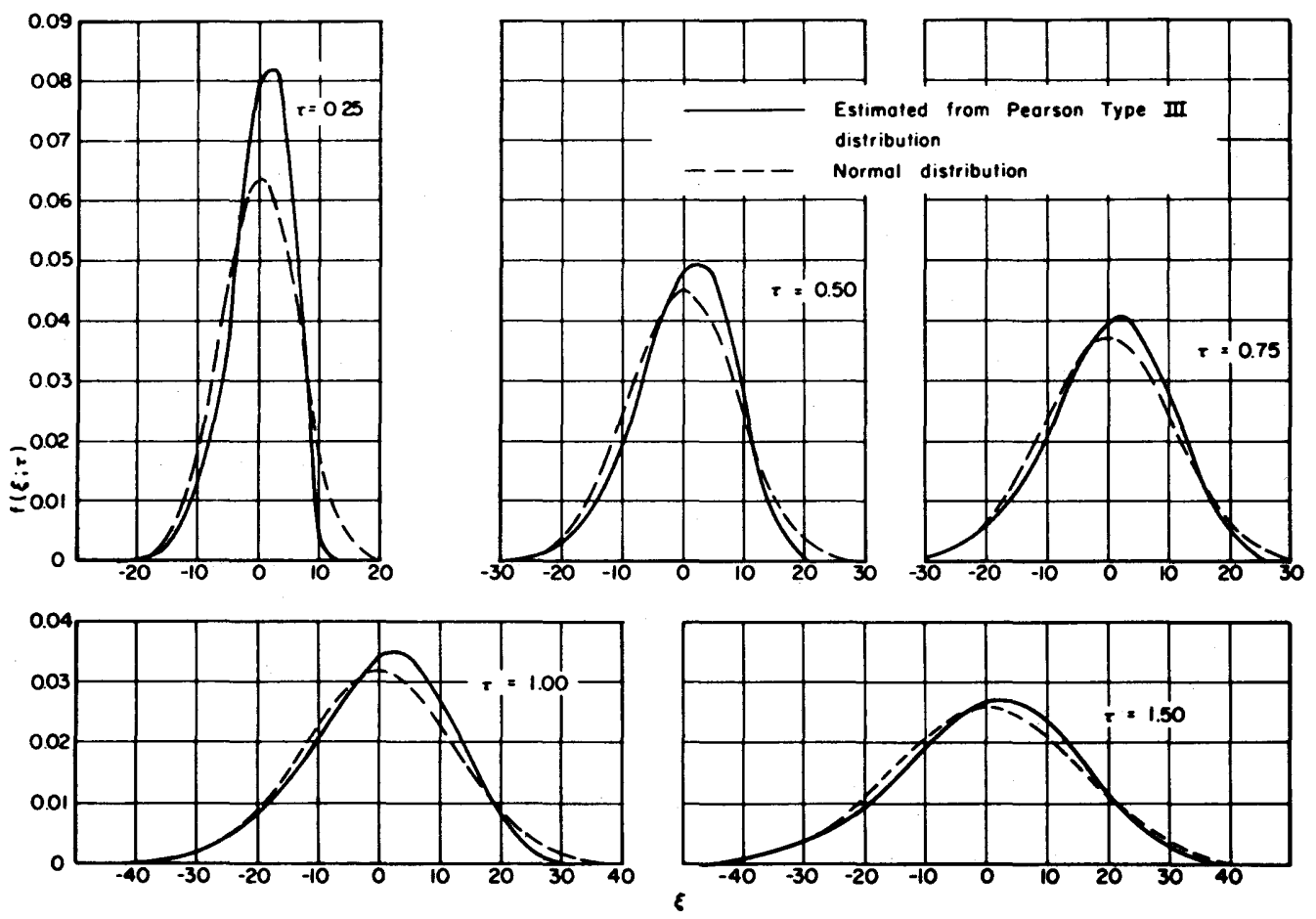

Figure 3-8. Estimated longitudinal concentration distributions at various dispersion times for $\kappa=0.42$ 
average for many particles, all released from the origin. Introducing again the Lagrangian correlation coefficient,

$$
\mathrm{R}_{\mathrm{u}_{\mathrm{L}}^{\prime}}\left(\tau^{\prime}\right)=\frac{\overline{\mathrm{u}_{\mathrm{L}}^{\prime}(\tau) \mathrm{u}_{\mathrm{L}}^{\prime}\left(\tau_{1}\right)}}{\overline{\mathrm{u}_{\mathrm{L}}^{\prime 2}}}
$$

where $\tau^{\prime}=\tau-\tau_{1}$, we obtain

$$
\frac{1}{2} \frac{\mathrm{d} \sigma_{\xi}^{2}(\tau)}{\mathrm{d} \tau}=\overline{\mathrm{u}_{L}^{\prime 2}} \int_{0}^{\tau} \mathrm{R}_{\mathrm{u}_{\mathrm{L}}^{\prime}}\left(\tau^{\prime}\right) \mathrm{d} \tau^{\prime}
$$

and finally

$$
\sigma_{\xi}^{2}(\tau)=\overline{2 u_{L}^{\prime 2}} \int_{0}^{\tau}\left(\tau-\tau^{\prime}\right) R_{u_{L}^{\prime}}\left(\tau^{\prime}\right) d \tau^{\prime} .
$$

In a turbulent shear flow it is to be expected that the Lagrangian correlation coefficient will vary not only with the lag time, $\tau^{\prime}$, but also with the position, $\eta$, from which particles are released. Where this is true, $R_{u_{L}^{\prime}}^{\prime}\left(\tau^{\prime}, \eta\right)$ should replace $R_{u}^{\prime}\left(\tau^{\prime}\right)$ in equation 3-60. In our particular case, where a uniformly distributed ensemble of particles is released instantaneously, equation 3-60 should be averaged over the depth of flow. In effect, this is accomplished by replacing $R_{u_{L}^{\prime}}^{\prime}\left(\tau^{\prime}\right)$ by the averaged coefficient

$$
\overline{\mathrm{R}}_{\mathrm{u}_{\mathrm{L}}^{\prime}}\left(\tau^{\prime}\right)=\int_{0}^{1} \mathrm{R}_{u_{\mathrm{L}}^{\prime}}\left(\tau^{\prime}, \eta\right) \mathrm{d} \eta
$$

The formulation and interpretation of $\bar{R}_{u_{L}^{\prime}}\left(\tau^{\prime}\right)$ in a turbulent shear flow will be considered in more detail later on. For the present we will only assume that such a formulation is possible.

Let us now consider the Lagrangian intensity of turbulence, $\overline{u_{L}^{\prime}}$. There are two ways in which $\overline{u_{L}^{\prime}}$ can be estimated. The first method is based on the concept of a fluid particle wandering randomly in a known velocity field as shown in the sketch below. The instantaneous velocity of the particle is

$$
u_{L}^{\prime}=\mu X(\eta)+\mu X^{\prime}
$$

If we neglect the fluctuating component $\mu X^{\prime}$ as being of minor importance, then we have

$$
\mathbf{u}_{\mathrm{L}}^{\prime} \approx \mu \mathrm{X}(\eta)
$$

so that the velocity of the particle at any time $\tau$ depends on its position only. If there is equal likelihood that the particle will wander anywhere in the flow field then the averages

and

$$
\overline{u_{L}^{\prime}{ }^{2}}=\frac{1}{T} \int_{0}^{T} u_{L}^{\prime 2} d \tau
$$

$$
\overline{u_{L}^{\prime}}=\int_{0}^{1}(\mu x)^{2} d \eta=\overline{(\mu x)^{2}}
$$

are equivalent, provided that the averaging time $\mathrm{T}$ is sufficiently long for the particle to sample the flow field. For the logarithmic velocity distribution,

$$
\overline{u_{L}^{\prime 2}}=\overline{(\mu \chi)^{2}}=\frac{36}{\kappa^{4}} \int_{0}^{1}(\ln \eta+1)^{2} \mathrm{~d} \eta=\frac{36}{\kappa^{4}} .
$$

For the finite difference approximation of the logarithmic velocity distribution used in the numerical solution with $\Delta \eta=0.05$,

$$
\overline{u_{L}^{\prime 2}}=\overline{(\mu \mathrm{X})^{2}}=\frac{36}{\kappa^{4}} \sum_{I=1}^{20}[\mathrm{U}(\mathrm{I})]^{2} \mathrm{DY}=\frac{34.1}{\kappa^{4}} .
$$

The second method of estimating $\overline{u_{1}^{\prime 2}}$ is based on the limiting solution of equation 3-60 for $\tau^{\prime} \longrightarrow 0$ from which we obtain

$$
\overline{u_{L}^{\prime 2}}=\operatorname{Lim}_{\tau \rightarrow 0} \frac{\sigma_{\xi}^{2}}{\tau^{2}}
$$

This is illustrated in Figure 3-9(a) for both $\sigma_{\xi}^{2}$ and $\sigma_{\xi}^{2}-2 \tau$. The latter is actually more appropriate for comparison with equation 3-62 because it represents the convective component of the variance. This is because the direct contribution to $\overline{u_{L}^{\prime 2}}$ by turbulence, i. e. $\mu X^{\prime}$, was neglected in the development leading to equation 3-62. The curves in Figure 3-9(a) seem to be approaching the values of $\overline{u_{L}^{\prime}{ }^{2}}$ specified by
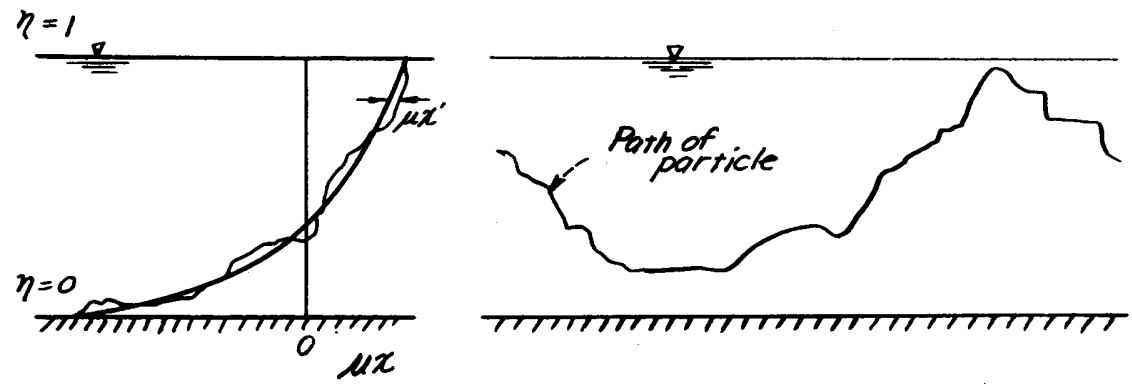

$\mu x$ 

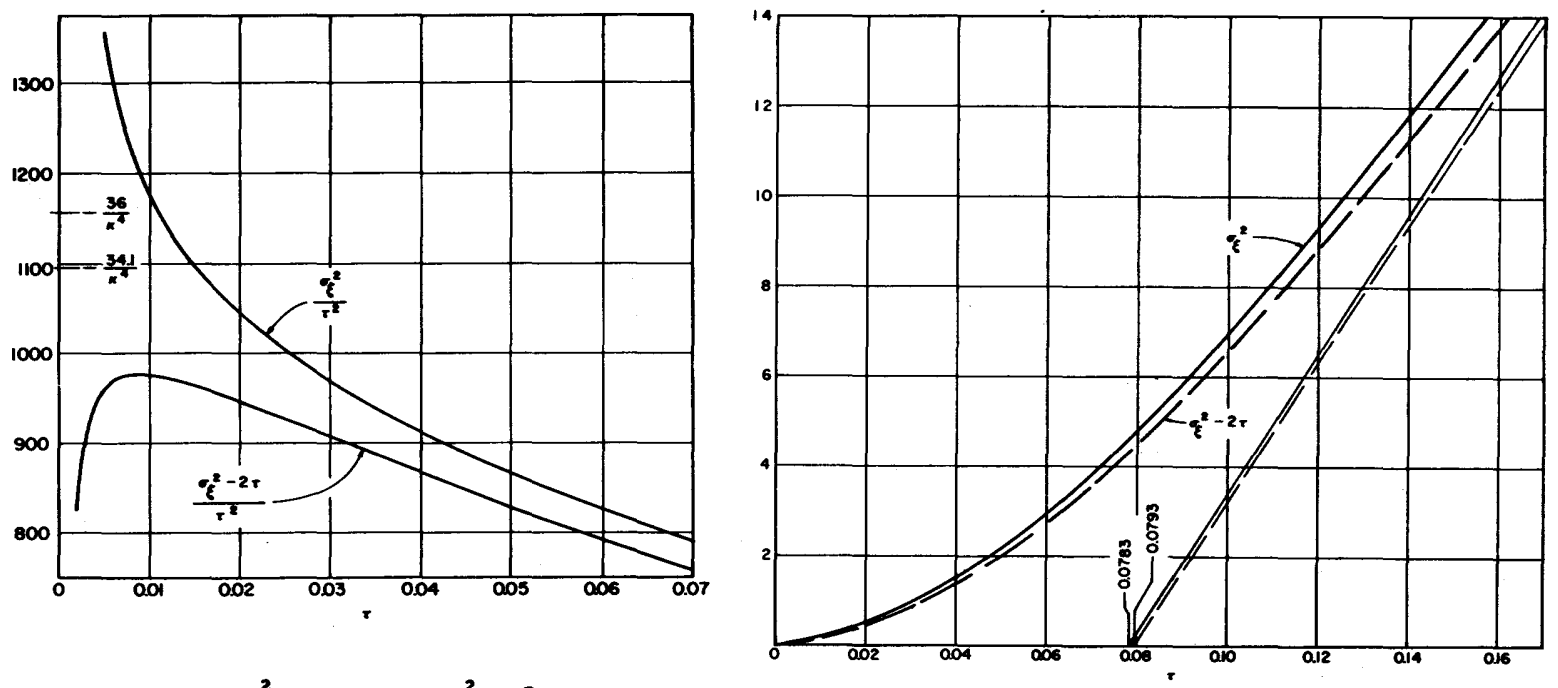

a. $\frac{\sigma_{\xi}^{2}}{\tau^{2}}$ and $\frac{\sigma_{\xi}^{2}-2 \tau}{\tau^{2}}$

b. $\sigma_{\xi}^{2}$ and $\sigma_{\xi}^{2}-2 \tau$

Figure 3-9. Behavior of the variance at very small dispersion times, $\kappa=0.42$

equation 3-62 as $\tau$ decreases, however, they veer away at extremely small values of $\tau$. A probable explanation for this is that the numerical solution breaks down in the immediate neighborhood of $\tau=0$.

In Figure 3-9(b) $\sigma_{\xi}^{2}$ and $\sigma_{\xi}^{2}-2 \tau$ are shown as functions of $\tau$ for very small dispersion times. Note the straight lines to the right which are the asymptotes and the $\tau$ intercepts of the asymptotes. The significance of the intercepts will become clear later.

Having evaluated $\overline{u_{L}^{\prime}}$, the Lagrangian integral scale $L_{\tau}$ can now also be determined. Equation 3-60 for $\tau>0.5$, when written for the convective component of the variance, reduces to

$\sigma_{\xi}^{2}(\tau)-2 \tau=2 \overline{u_{L}^{\prime 2}} L_{\tau} \tau-2 \overline{u_{L}^{\prime 2}} \int_{0}^{\infty} \tau \bar{R}_{u_{L}^{\prime}}(\tau) d \tau$.

Noting that the term farthest to the right is a constant, taking the derivative with respect to $\tau$ of both sides, and solving for $L_{\tau}$, we obtain

$$
L_{\tau}=\frac{1}{2 \overline{u_{L}^{\prime 2}}} \frac{d}{d \tau}\left[\sigma_{\xi}^{2}-2 \tau\right]=\frac{K_{\xi}-1}{\overline{(\mu x)^{2}}} .
$$

Equation 3-65, when evaluated with the results of the numerical solution for the logarithmic velocity distribution case with $\Delta \eta=0.05$ and $\tau>0.5$, gives $L_{\tau}=0.0703$. Noting further that equation 3-64 is of the form $y=a x+b$, the $x$ intercept of which is $-b / a$, the significance of the $\tau$ intercepts in Figure 3-9(b) emerges: The intercepts indicate the value of the first moment,

$$
\bar{\tau}_{1}=\frac{1}{L_{\tau}} \int_{0}^{\infty} \tau \bar{R}_{u_{L}^{\prime}}(\tau) \mathrm{d} \tau
$$

of the Lagrangian correlation coefficient.

The form of the Lagrangian correlation coefficient was determined, using numerical differentiation methods, from the relationship

$$
\bar{R}_{u_{L}^{\prime}}(\tau)=\frac{1}{2 \overline{u_{L}^{\prime}}} \frac{d^{2} \sigma_{\xi}^{2}}{d \tau^{2}}=\frac{1}{\overline{(\mu \mathrm{X})^{2}}} \frac{d K_{\xi}}{d \tau}
$$

Here again, the results of the numerical solution were used. The results of this calculation are shown in Figure 3-10 on both rectangular and semilog coordinates. The semilog plot shows that $\bar{R}_{u_{L}^{\prime}}^{\prime}(\tau)$ is exponential in form except in the range $0<T<0.1$. By numerically integrating $\bar{R}_{u_{L}^{\prime}}^{\prime}(\tau)$,

and

$$
L_{\tau}=\int_{0}^{\infty} \bar{R}_{u_{L}^{\prime}}(\tau) d \tau=0.0706
$$

$$
\bar{\tau}_{1}=\frac{1}{L_{\tau}} \int_{0}^{\infty} \tau \bar{R}_{u_{L}^{\prime}}(\tau) \mathrm{d} \tau=0.0782
$$

were determined. These numerical values agree very closely with the one from equation 3-65 and both of the $\tau$ intercepts in Figure 3-9(b). 

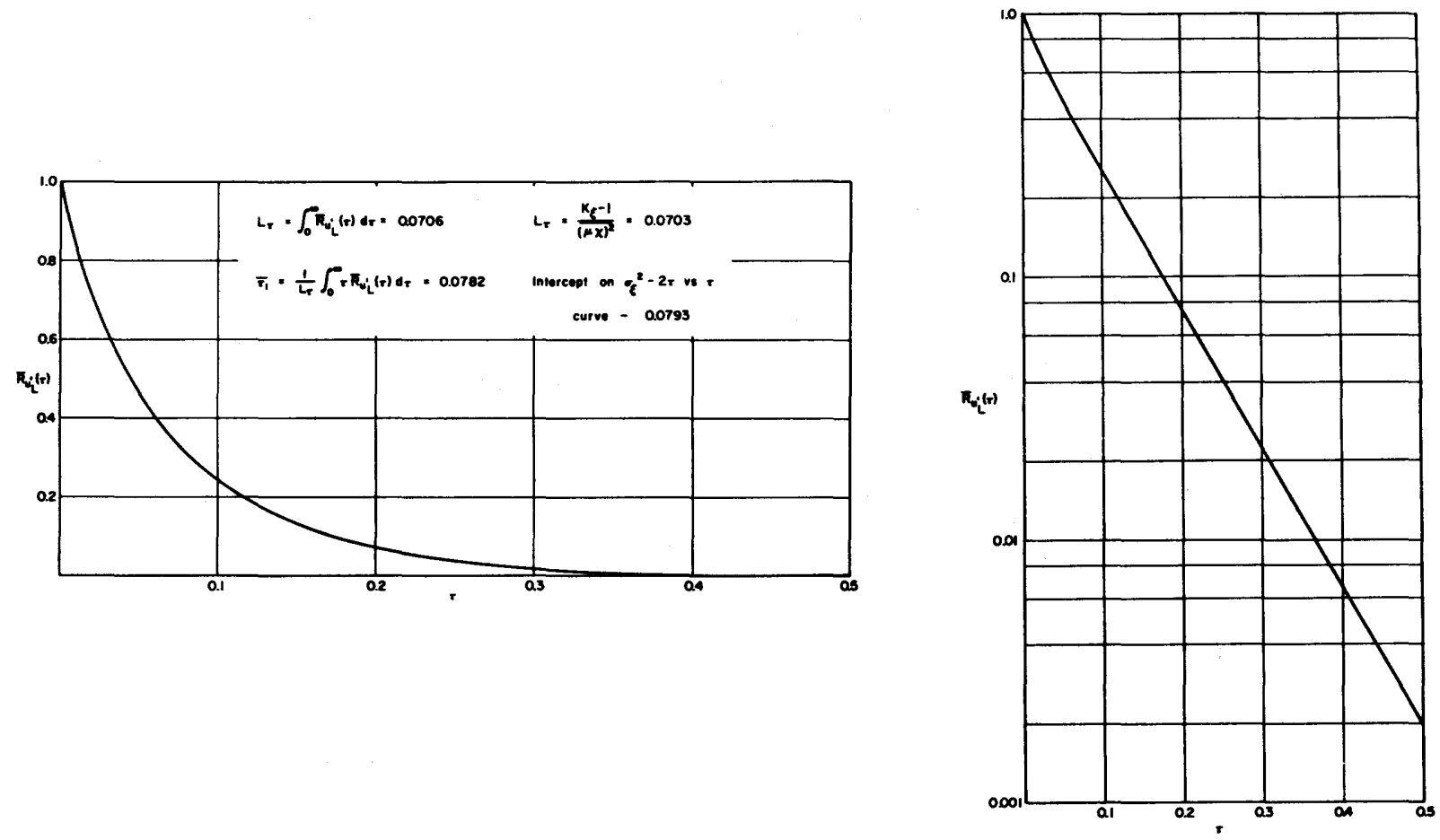

Figure 3-10. Average Lagrangian correlation coefficient as a function of dispersion time

For comparison, the values of $\overline{(\mu \mathrm{X})^{2}}, \mathrm{~L}_{\tau}$ and $\bar{\tau}_{1}$ as determined with equations 3-62(a), 3-65 and 3-64 from the numerical solutions with $\Delta \eta=0.05$ of the logarithmic and parabolic velocity distribution cases, are listed in Table 3-6.

Let us turn now to a more precise formulation of the Lagrangian correlation coefficient in a turbulent shear flow. If we continue to assume that the fluctuating component of the velocity $\mu X^{\prime}$ is of minor importance in comparison to $\mu \times(\eta)$ in causing longitudinal dispersion, then the instantaneous velocity of a fluid particle which at time $\tau$ is located at position $\eta$ can be represented as $\mu \chi(\tau, \eta)$. If after an arbitrary time interval $\tau^{\prime}$ the vertical displacement of the particle from its initial position is $\eta^{\prime}$, then the instantaneous velocity of the particle at time $\tau+\tau^{\prime}$ is $\mu \times\left(\tau+\tau^{\prime}, \eta+\eta^{\prime}\right)$. For an ensemble of particles that is initially uniformly distributed over the entire depth of flow, the correlation coefficient is

$$
\overline{\mathrm{R}}_{\mathrm{u}_{\mathrm{L}}^{\prime}}\left(\tau^{\prime}\right)=\frac{1}{\overline{(\mu \mathrm{x})^{2}}} \int_{0}^{1} \overline{\mu \mathrm{x}(\tau, \eta) \mu \mathrm{x}\left(\tau+\tau^{\prime}, \eta+\eta^{\prime}\right)} \mathrm{d} \eta .
$$

Because $\mu \chi(\tau, \eta)$ is a constant for a given $\eta$, an equivalent formulation would be

$$
\overline{\mathbf{R}}_{u_{L}^{\prime}}\left(\tau^{\prime}\right)=\frac{1}{(\mu \mathrm{x})^{2}} \int_{0}^{1} \mu \times(\eta) \int_{-\eta}^{1-\eta} \mu \times\left(\eta+\eta^{\prime}\right) \mathrm{f}_{\eta}\left(\eta^{\prime} ; \tau^{\prime}\right) \mathrm{d} \eta^{\prime} \mathrm{d} \eta
$$

where $f_{\eta}\left(\eta^{\prime} ; \tau^{\prime}\right)$ is the probability density function for the displacement of a particle from $\eta$ to $\eta+\eta^{\prime}$ during the time interval $\tau^{\prime}$, and the boundary conditions for $f_{\eta}\left(\eta^{\prime} ; \tau^{\prime}\right)$ are $\partial f_{\eta} / \partial \eta=0$ at $\eta=0$ and $\eta=1$. The sketch on the following page will help to clarify the meaning of equation $3-68$. It is likely that

TABLE 3-6. LAGRANGIAN TURBULENCE CHARACTERISTICS FOR FLOWS WITH LOGARITHMIC AND PARABOLIC VELOCITY DISTRIBUTIONS

\begin{tabular}{|cccc|}
\hline Velocity Distributions & $\overline{(\mu \mathrm{X})^{2}}$ & $\mathrm{~L}_{\tau}$ & $\overline{\tau_{1}}$ \\
\hline Logarithmic & $34.1 / \kappa^{4}$ & 0.0703 & 0.0793 \\
Parabolic & $28.7 / \kappa^{4}$ & 0.0955 & 0.0996 \\
\hline
\end{tabular}




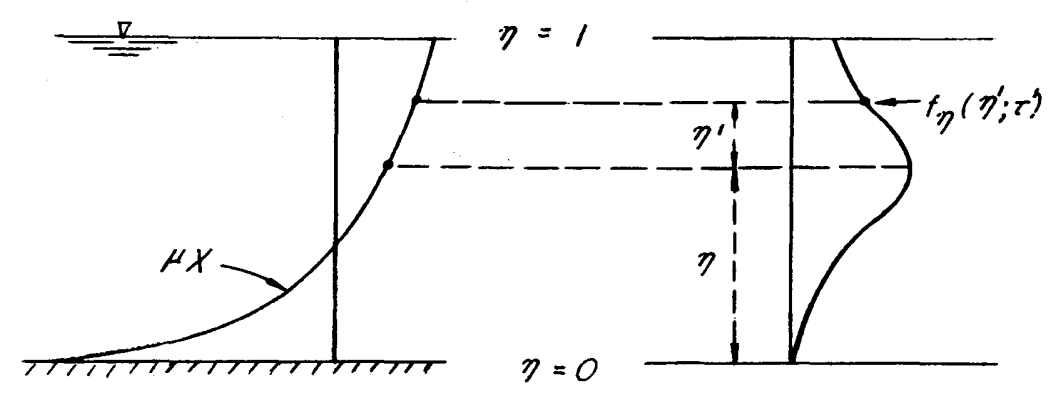

for very small $\tau^{\prime}, f_{\eta}\left(\eta^{\prime} ; \tau^{\prime}\right)$ will be very closely related to the diffusivity function $\psi$, i.e., normally distributed with mean $\eta^{\prime}=0$ and variance $\sigma^{2}=2 \psi \tau^{\prime}$. For intermediate $\tau^{\prime}, f_{\eta}\left(\eta^{\prime} ; \tau^{\prime}\right)$ is unknown, but for large $\tau^{\prime}, f_{\eta}\left(\eta^{\prime} ; \tau^{\prime}\right)$ will be uniformly distributed.

Formulating the convective component of a longitudinal dispersion coefficient with $\bar{R}_{u_{L}^{\prime}}\left(\tau^{\prime}\right)$, as defined in equation $3-68$, we obtain

$$
\begin{aligned}
\mathrm{K}_{\mathrm{C}} & =\frac{1}{2} \frac{\mathrm{d}}{\mathrm{d} \tau}\left[\sigma_{\xi}^{2}-2 \tau\right]=\overline{(\mu \mathrm{X})^{2}} \int_{0}^{\tau} \overline{\mathrm{R}}_{\mathrm{u}_{\mathrm{L}}^{\prime}}\left(\tau^{\prime}\right) \mathrm{d} \tau^{\prime} \\
& =\int_{0}^{1} \mu \mathrm{x}(\eta) \int_{-\eta}^{1-\eta} \mu \mathrm{x}\left(\eta+\eta^{\prime}\right) \int_{0}^{\tau} \mathrm{f}_{\eta}\left(\eta^{\prime} ; \tau^{\prime}\right) \mathrm{d} \tau^{\prime} \mathrm{d} \eta^{\prime} \mathrm{d} \eta .
\end{aligned}
$$

It is interesting to speculate on how equation 3-69 relates to Elder's (1959) equation 2-26,

$$
\operatorname{Lim}_{\tau \rightarrow \infty} \mathrm{K}_{\mathrm{C}}=-\int_{0}^{1} \mu \mathrm{x} \int_{0}^{\eta} \frac{1}{\psi} \int_{0}^{\eta} \mu \mathrm{x} \mathrm{d} \eta \mathrm{d} \eta \mathrm{d} \eta
$$

to which it bears at least a superficial resemblance.

All things considered, the theory of diffusion by continuous movements appears to be less suitable than the Eulerian theory for describing the longitudinal dispersion process in a turbulent shear flow. However, the comparison between the two theories is useful because it demonstrates the generality of Taylor's theory, and also because it helps to clarify the relationship between the Eulerian and Lagrangian descriptions of dispersion in turbulent shear flow.

\section{c. Results for suspended sediment when}

$\gamma=0$ - When $\beta>0$, the dispersant tends to settle toward the bed and the mathematical model simulates the longitudinal dispersion of sediment particles. The main purpose of this section is to demonstrate the influence of the fall velocity term, $\beta=\nu_{S} / 6=V_{S} / \kappa U_{T}$, and the bed absorbency factor, $\alpha$, in cases where no entrainment of sediment from the bed is permitted. The range of $\beta$ was selected to simulate silt-size sediment particles in rough-boundary flow. When $\alpha=1$, the bed behaves as a completely absorbing boundary. All particles which come into contact with the bed remain there and are thenceforth eliminated from the dispersion process, provided that attention is restricted to the suspended particles. When $\alpha=0$, the bed behaves as a completely reflecting boundary so that no deposition is permitted. All particles which come into contact with the bed are immediately resuspended in the bottom increment of the flow. When $0<\alpha<1$, the bed behaves as a partially absorbing boundary and the value of $\alpha$ represents the fraction of particles striking the bed that are retained there. The remaining fraction, $1-\alpha$, are reflected.

All of the results reported in this section are for flows with a logarithmic velocity distribution in which $\kappa=0.42$. Furthermore, because no entrainment is permitted $(\gamma=0)$, and attention is restricted to the suspended particles, solutions of equation 3-7 are independent of equation 3-11 so that the results are independent of the mean velocity term $\mu=(6 / \kappa)\left(\bar{U} / U_{\tau}\right)$. If entrainment is permitted and/or the deposited particles are included in the longitudinal distributions, this is no longer true. Such cases are taken up in later sections.

Figure 3-11 shows vertical distributions of suspended sediment averaged over the length of the dispersing cloud, for different values of $\beta$ for both the absorbing and the reflecting boundary conditions. The distributions, which are uniform at $\tau=0$, approach a state of equilibrium with increasing $\tau$, attaining it at about $\boldsymbol{T}=\mathbf{0 . 5}$. All of the solutions shown are for the equilibrium situation. As anticipated, the gradients of the distributions become more pronounced as the fall velocity term, $\beta$, increases. Note that the solution for the reflecting boundary case, $\alpha=0$, is apparently identical to equation $2-44$, which is the familiar vertical concentration distribution function for the steady state equilibrium condition where the deposition and entrainment rates are equal. Solutions for $0<\alpha<1$ fall between those for the limiting cases $\alpha=0$ and $\alpha=1$.

When $\alpha=0$ the total amount of sediment retained in suspension, $m_{0}(\tau)$, remains constant. In the absorbing boundary case, when $\alpha=1, m_{0}(\tau)$ decreases with dispersion time as shown in Figure 3-12. The decrease can be represented empirically by the exponential function

$$
m_{0}(\tau)=e^{-\lambda \tau}
$$




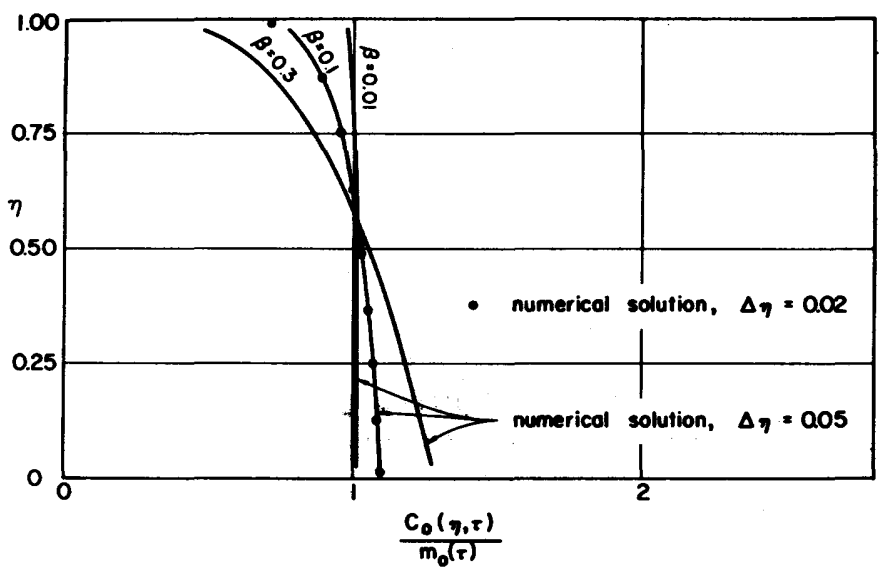

a. Absorption condition, $\alpha=1$

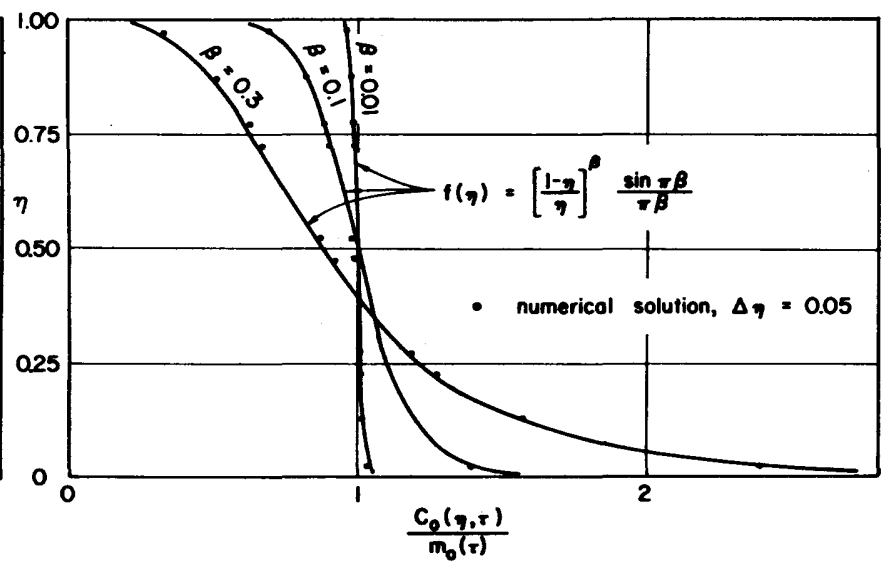

b. Reflection condition, $\alpha=0$

Figure 3-11. Vertical distributions of suspended sediment when $\tau \geq 0.5$ for particles having different terminal fall velocities

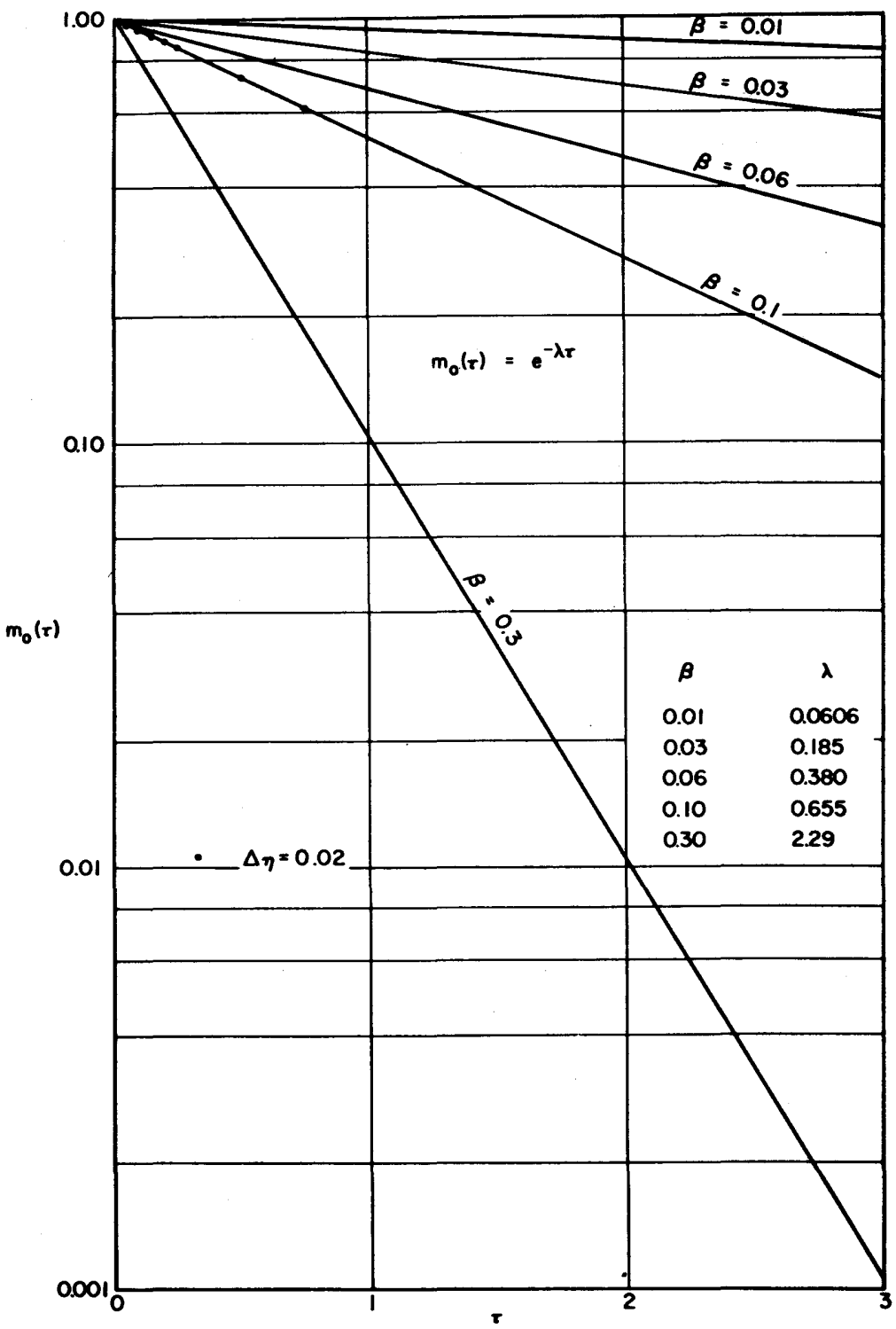

Figure 3-12. Fraction of sediment retained in suspension when $\alpha=1$ as a function of dispersion time for particles having different terminal fall velocities 
If $\mathrm{m}_{0}(\tau)$ is interpreted as the probability that a randomly chosen particle is still in suspension at time $\tau$, then $1-m_{0}(\tau)$ represents the probability that the duration of the period of retention in suspension is equal to or less than $\tau$. Furthermore

$$
f(\tau)=\frac{\partial}{\partial \tau}\left[1-m_{0}(\tau)\right]=\lambda e^{-\lambda \tau}
$$

is the probability density function for the duration of the retention period, from which the mean retention time

$$
\bar{\tau}=\int_{0}^{\infty} \tau f(\tau) d \tau=\frac{1}{\lambda}
$$

can be obtained. Thus, the coefficient $\lambda$ can be interpreted as the mean rate at which particles settle out of suspension.

In Figure $3-13, \lambda$ is shown as a function of $\beta$. The results obtained from the numerical solutions with $\alpha=1$, shown as the solid black circles, can be represented by the empirical relationship

$$
\lambda=6 \beta(0.9 \beta+1) .
$$

The single black triangle represents a solution for the case of a partially absorbing boundary, with $\alpha=0.5$. Although $\lambda$ decreases with $\alpha$, the relative values of $\lambda$ for $\beta=0.1$, and $\alpha=1,0.5$ and $0(\lambda=0$ when $\alpha=0$ ) indicate that the relationship is not linear. It is noteworthy that the solution of Dobbins' (1944) equation 3-22, which was derived for the case of a uniformly distributed eddy diffusivity, leads to values of $\lambda$ which agree very closely with equation 3-73. The implication here is that whereas the rate at which sediment settles out of suspension is presumably a function of the average vertical eddy diffusivity $D$, it may be insensitive to the diffusivity distribution function $\psi$.

Focusing attention now on the overall transport rate of the suspended particles, Figure 3-14 shows some examples of the mean longitudinal displacement, $\bar{\xi}_{S}$, of the suspended particles from the reference plane $(\xi=0)$ which is moving at the mean flow velocity, as a function of dispersion time. It is helpful to recall here that the displacement $\bar{\xi}_{\mathrm{S}}$, is measured in units of flow depths. The results in Figure 3-14 show that the mean position of the suspended particles advances more rapidly than the flow when the particles are absorbed by the bed $(\alpha=1)$, and less rapidly when the particles are reflected by

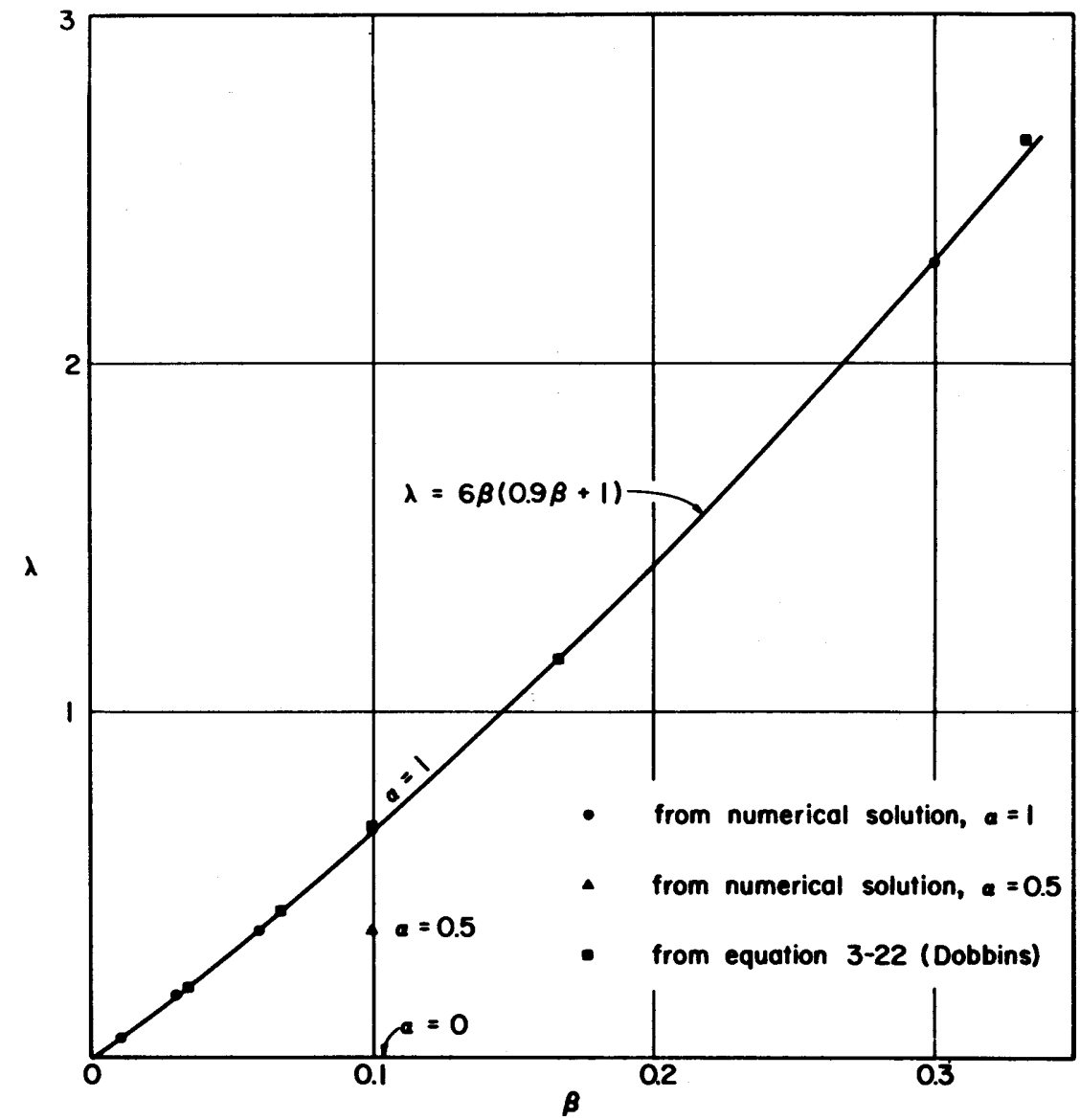

Figure 3-13. Mean rate at which particles settle out of suspension as a function of terminal fall velocity 


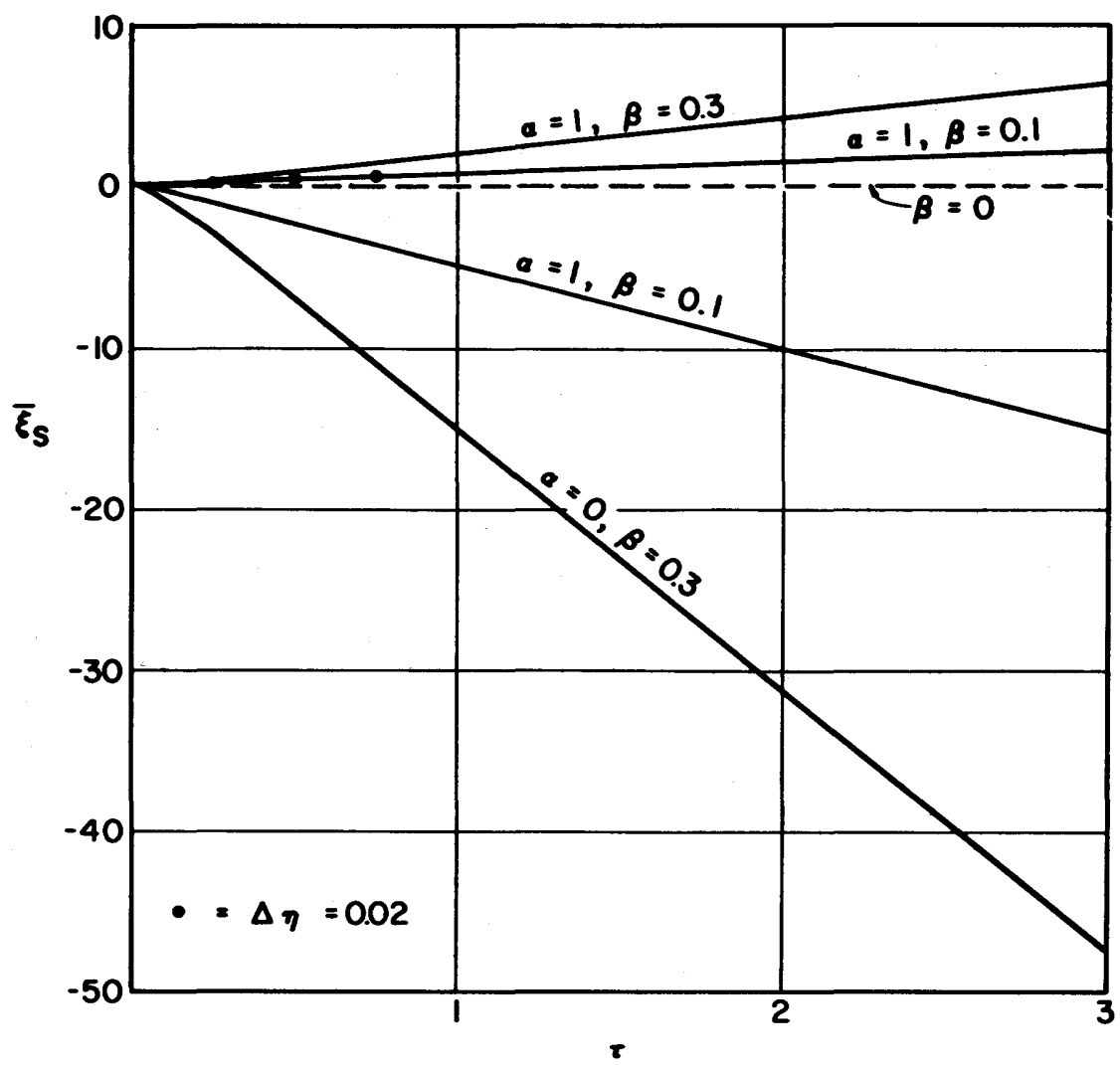

Figure 3-14. Mean longitudinal displacement, in a coordinate system moving at the mean flow velocity, of the suspended sediment as a function of dispersion time

the bed $(\alpha=0)$. Considering that the vertical concentration gradients are in both cases weighted toward the bottom where the velocity is slower, the result for $\alpha=1$ at first seems surprising. Upon further consideration, however, the reason becomes clear. The particles which settle out of suspension first tend to be those which were initially suspended in the slower moving flow near the bed. Conversely, those retained in suspension the longest tend to be the ones with the longest history of suspension in the upper region of the flow where velocities exceed the average. The rates of displacement, indicated by the slopes of the curves, increase slightly in absolute value at small dispersion times, reaching a limiting value at about $\tau=0.5$, when equilibrium vertical distributions are attained. The limiting rates $d \bar{\xi}_{S} / d_{\tau}$, are shown as functions of $\beta$ in Figure 3-15. The instantaneous average velocity of the suspended particles,

$$
\bar{\mu}_{\mathrm{s}}=\frac{1}{\mathrm{~m}_{0}} \int_{0}^{1} \mu \times \mathrm{C}_{0} \mathrm{~d} \eta,
$$

is shown also for comparison. When $\alpha=0$, and all the particles remain in suspension, $\bar{\mu}_{S}$ and $\mathrm{d} \bar{\xi}_{S} / \mathrm{d} \tau$ are equal. However, when $\alpha=1$ and deposition is occurring so that the population of suspended particles is continually decreasing, $\bar{\mu}_{\mathrm{S}}$ is less than $\mathrm{d} \bar{\xi}_{\mathrm{S}} / \mathrm{d} \tau$ because $\bar{\mu}_{\mathrm{S}}$ does not depend on the previous history of the suspended particles in the manner that $d \bar{\xi}_{S} / d \tau$ does. The results shown for $\alpha=0.5$ and 0.9 when $\beta=0.1$ give an indication of the behavior of $\mathrm{d} \bar{\xi}_{\mathrm{S}} / \mathrm{d} \tau$ and $\bar{\mu}_{\mathrm{S}}$ when the bed is a partially absorbing boundary.

Figure 3-16 which shows the mean displacement of the suspended particles from the source in a flow with mean velocity $\mu=100$ helps to place Figures 3-14 and 3-15 in perspective. The velocity $\mu=100$ corresponds to $\bar{U} / U_{\tau}=7$ which in turn is indicative of flow near a very rough boundary except at small Reynolds numbers.

Some examples of the variance, $\sigma_{S}^{2}$, of the longitudinal distribution of the suspended particles as a function of dispersion time are shown in Figure 3-17. The form of the relationships for the suspended particles resembles that for $\beta=0$ in that the slope of the curves increases initially and reaches a limiting value at about $\tau=0.5$. Here again the results for $\alpha=0$ and $\alpha=1$ show opposite trends. When $\alpha=0$ the degree of dispersion tends to increase as $\beta$ increases, reflecting the combined effect of the vertical distribution of suspended particles and the velocity distribution. However, when $\alpha=1$, the particles which are retarded in the slower moving 


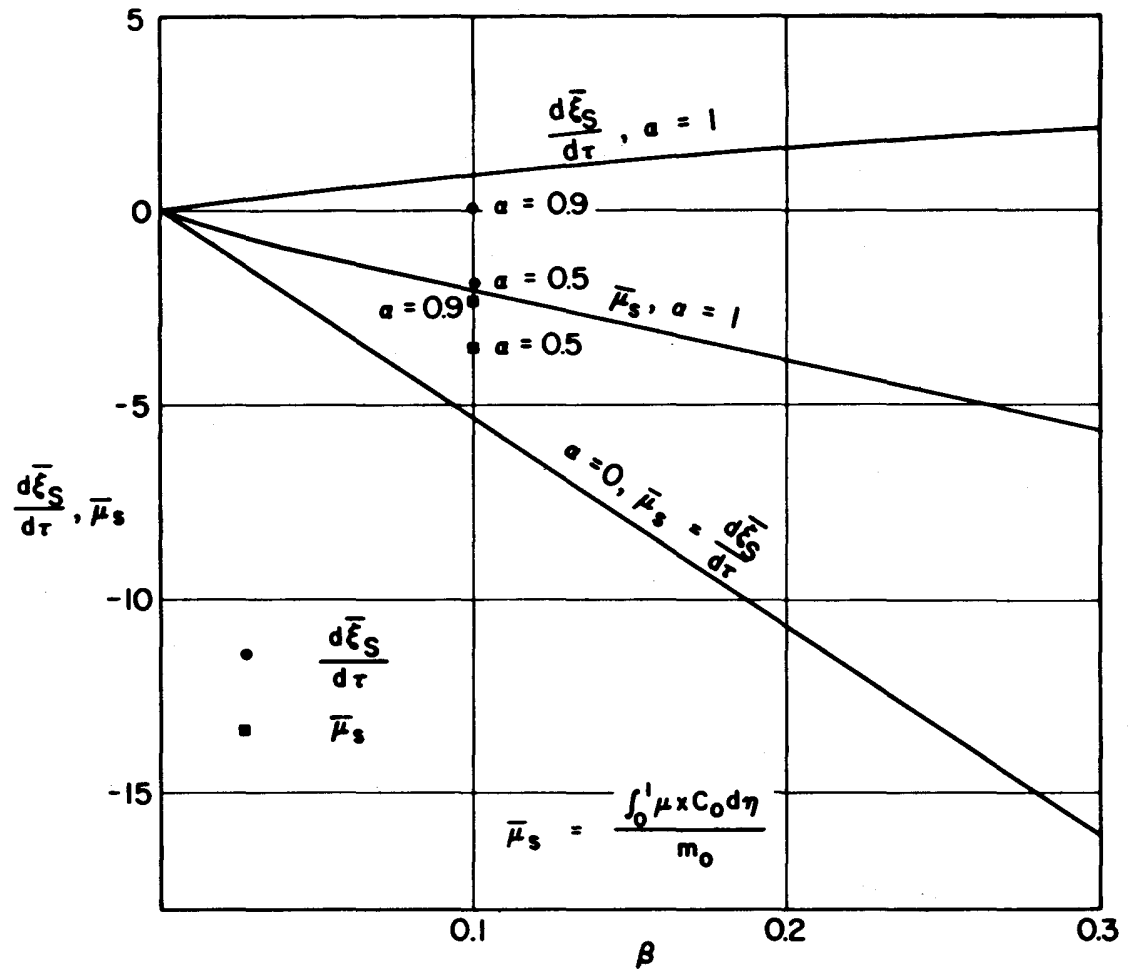

Figure 3-15. Limiting mean rate of longitudinal displacement, relative to mean flow velocity, of suspended sediment as a function of terminal fall velocity for $\tau \geq 0.5$

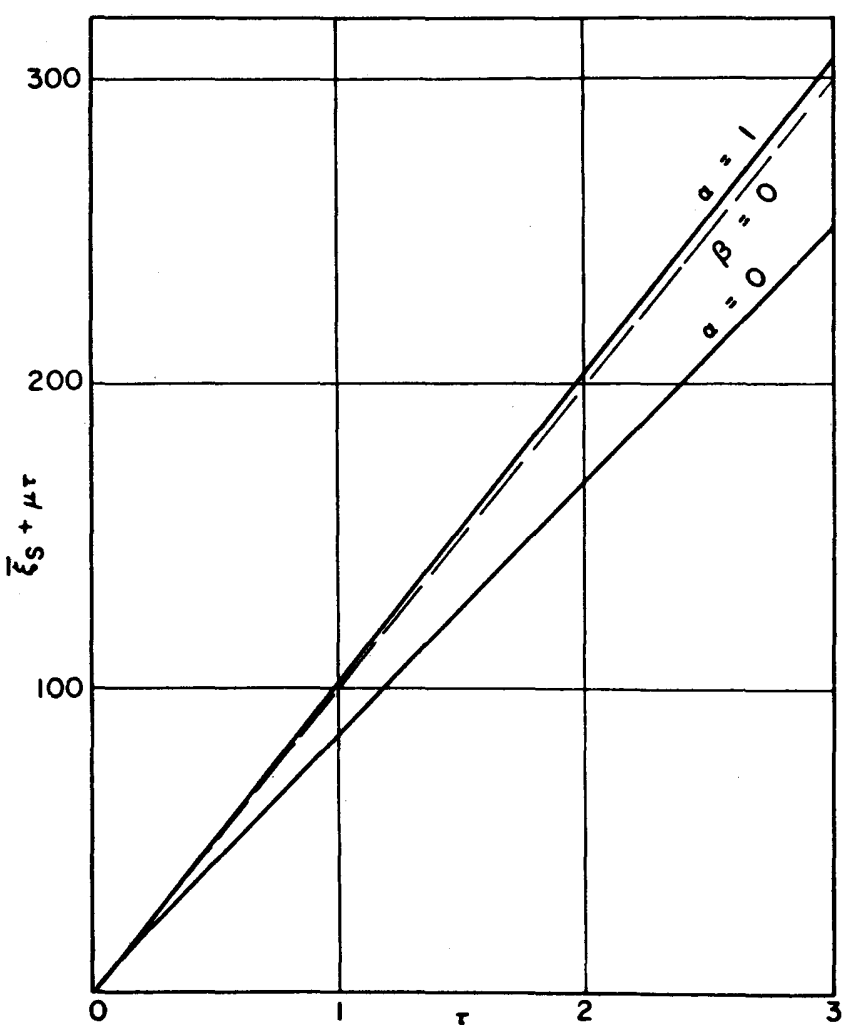

Figure 3-16. Mean longitudinal displacement, from the source, of suspended sediment as a function of dispersion time in a flow with $\bar{U} / U_{\tau}=7$ and $\beta=0.3$ 


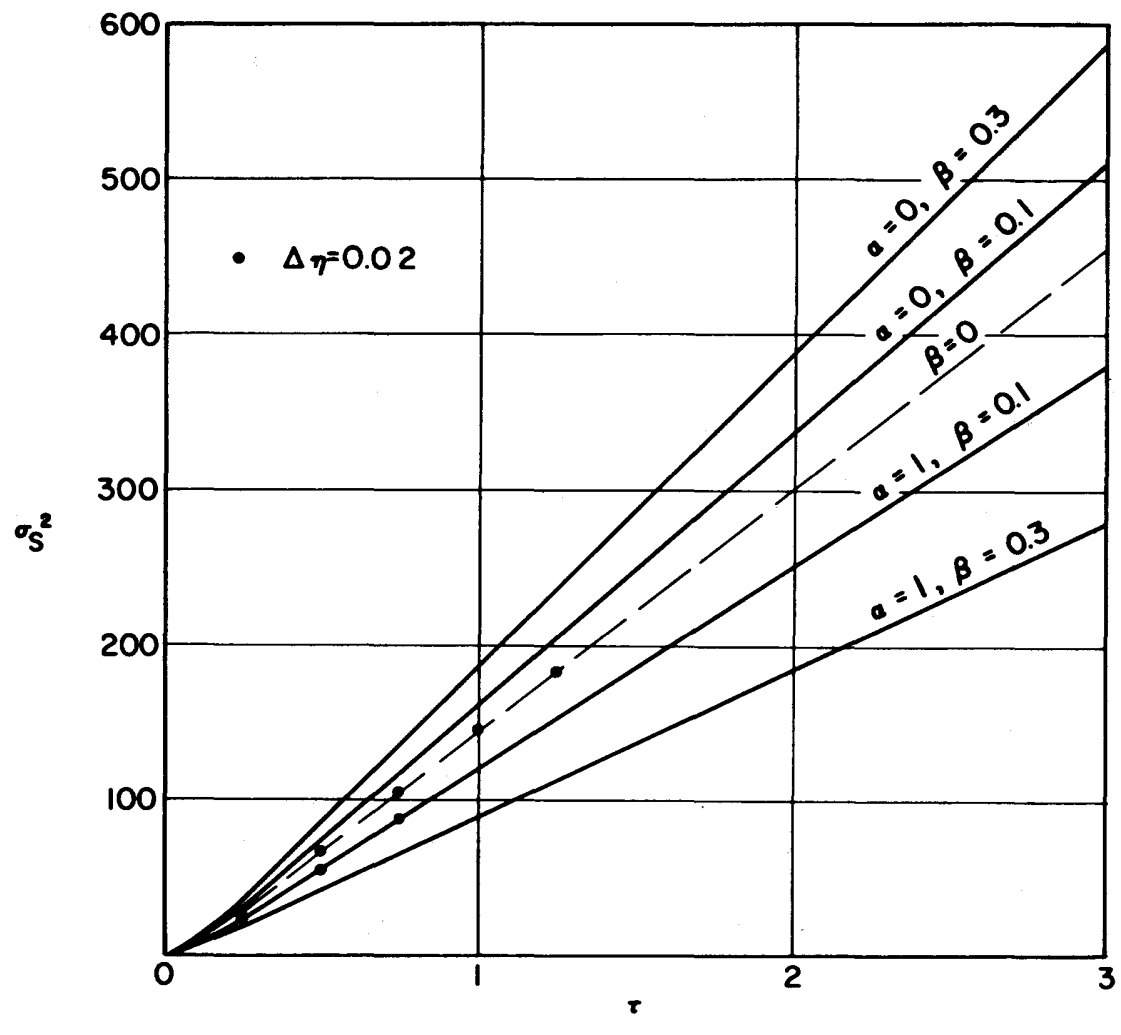

Figure 3-17. Variance of longitudinal distribution of suspended sediment as a function of dispersion time

flow near the bed and are contributing the most to the variance, tend to settle out of suspension.

The relative longitudinal dispersion coefficients, $\mathrm{K}_{\xi}(\beta) / \mathrm{K}_{\xi}(0)$, for the suspended sediment are given as functions of $\beta$ for both $\alpha=1$ and $\alpha=0$ in Figure 3-18. These are based on the asymptotic slopes of the $\sigma_{S}^{2}$ versus $\tau$ curves. The results for $\alpha=0.5$ and 0.9 when $\beta=0.1$ provide an additional indication of how the rate of dispersion varies with the bed absorbency factor. For comparison, Elder's (1959) equation 2-47, which was derived as a rough approximation of the reflecting boundary $(\alpha=0)$ case is also shown on Figure $3-18$. The reason why it diverges from the results of the numerical solution for $\alpha=0$ is not entirely clear. Possibly it is because Elder assumed the vertical distribution of particles to be the same throughout the entire length of the dispersing cloud.

In Figure 3-19, the $\tau$ intercepts, $\bar{\tau}_{1}$, obtained by extrapolating the asymptotic $\sigma_{S}^{2}$ versus $\tau$ relationships, are shown as functions of $\beta$. The influence of $\beta$ on $\bar{\tau}_{1}$ is not very pronounced, particularly when $\alpha=0$.

Some examples of the variation of the skew coefficient with respect to dispersion time are presented in Figure 3-20. When $\alpha=1$, the results for all values of $\beta$ ranging from 0 to 0.3 fall between the $\beta=0$ and the $\beta=0.3, \alpha=1$ curves. Therefore, the skew coefficient is quite insensitive to $\beta$ in the absorbing boundary case. When $\alpha=0$, however, the skewness decreases significantly with an increase in $\beta$.

The results of the numerical solutions also included the distributions of $\bar{\xi}_{S}(\eta, \tau), \sigma_{S}^{2}(\eta, \tau)$ and $\mathrm{S}_{\mathrm{S}}(\eta, \tau)$, with respect to $\eta$. An examination of these results, which is not presented here, showed them to be qualitatively but not necessarily quantitatively similar to the results given in Figure 3-7 for $\beta=0$.

Taking all of the results presented in this section together, it would seem that in a qualitative sense the longitudinal dispersion process for suspended sediment particles is not greatly different from the process for a neutrally-buoyant dispersant for which $\beta=0$. To be sure, there are significant quantitative differences, but these tend to be differences in degree rather than fundamental differences. In the next section, where the re-entrainment of deposited particles is considered, some fundamental differences come to light.

d. Results for suspended sediment when $\alpha=1$ and $\gamma>0$ - When $\gamma>0$, particles resting on the bed of the channel may be entrained in the flow. In this section the effect of the re-entrainment of deposited particles on the longitudinal distribution of suspended sediment is examined. In all cases, the bed is treated as a completely absorbing boundary, i. e., $\alpha=1$. As in the last section, all of the results are for flows with a logarithmic velocity distribution in which $\kappa=0.42$. Unlike the last section, the results 


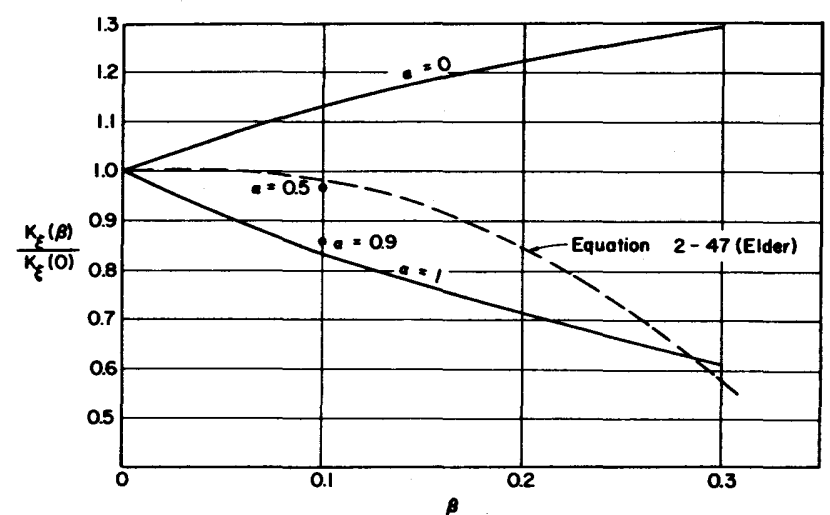

Figure 3-18. Relative longitudinal dispersion coefficient for suspended sediment as a function of terminal fall velocity

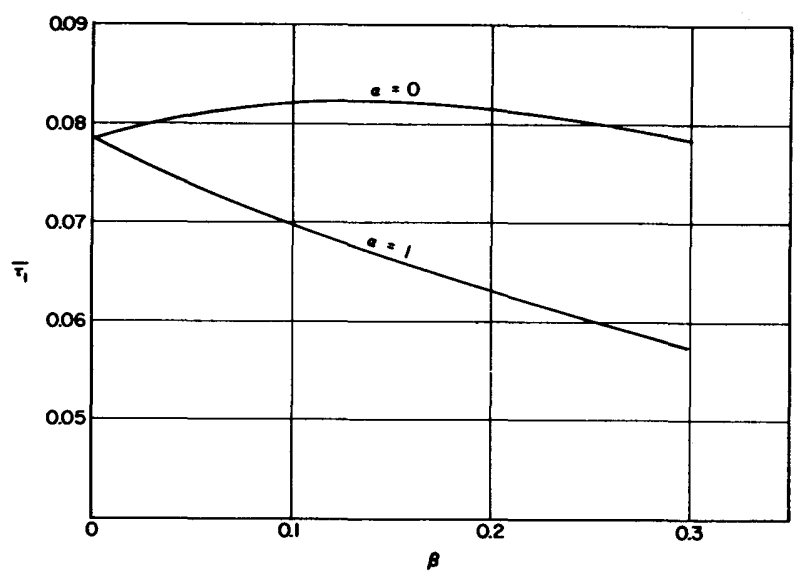

Figure 3-19. Intercept on $\sigma_{\mathrm{S}}^{2}$ vs. $\tau$ curve for suspended sediment as a function of terminal fall velocity

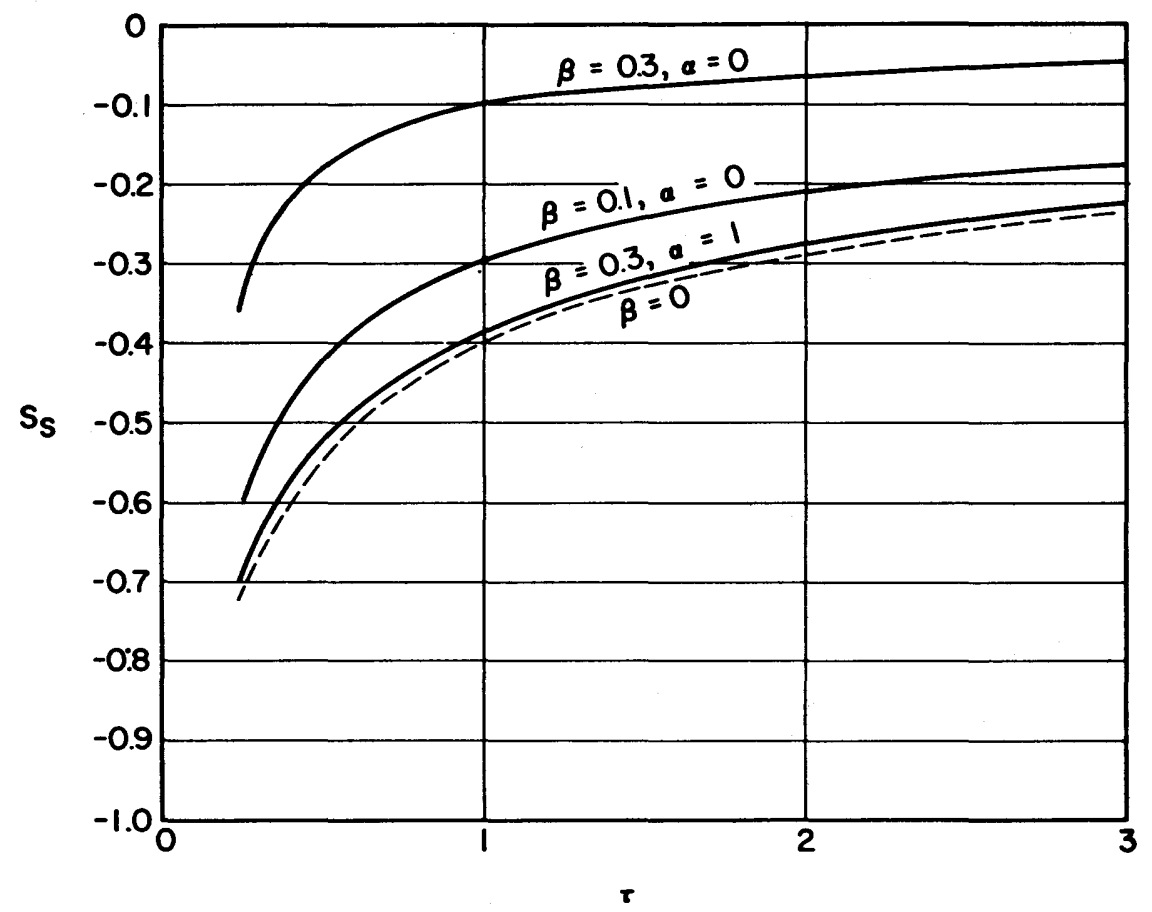

Figure 3-20. Skew coefficient for longitudinal distributions of suspended sediment as a function of dispersion time 
based on moments greater than $p=0$ are no longer independent of the mean velocity term, $\mu$.

Before launching into a description of the results, it would be well to examine more closely some of the attributes of the entrainment process. Equation 3-6 in a stationary frame of reference and with no deposition, reduces to

$$
\frac{d W}{d \tau}=-\gamma W
$$

which is merely a statement defining the rate of entrainment. If the probability of entrainment is, (1) the same for all particles, and (2) independent of the length of time that a particular particle has remained at rest, the solution of equation 3-74, giving the fraction of the particles remaining in an arbitrary control area at time $\tau$, is

$$
\frac{W(\tau)}{W(0)}=e^{-\gamma \tau}
$$

This result may also be interpreted as the probability that a given particle will not be entrained during the time interval, $(0, \tau)$. The probability that the particle will be entrained, which is equivalent to the probability that the time which the particle remains at rest is equal to or less than $\tau$, is then

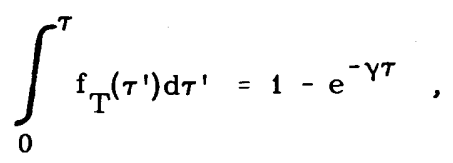

where $f_{T}\left(\tau^{\prime}\right)$ is the probability density function of the random variable $T$ which describes the duration of the rest period. Differentiating, we get the exponential probability density function,

$$
f_{T}(\tau)=\gamma e^{-\gamma \tau} \text {. }
$$

This function was used by Hubbell and Sayre (1964) and Einstein (1937) to express rest-period durations in deriving a stochastic model for the longitudinal dispersion of bed-material particles. From equation 3-76 can be obtained the mean rest period duration between successive entrainments,

$$
\bar{\tau}=\int_{0}^{\infty} \tau f_{T}(\tau) d \tau=\frac{1}{\gamma} .
$$

Provided the restriction is observed that coverage of the bed not exceed a single layer of particles, the validity of equation 3-76 is not affected by the occurrence of deposition. If the bed is covered by more than a single layer of particles, $\gamma$ will tend to decrease as the cover thickness increases, but the general form of equation 3-76 should remain valid provided that there is vertical mixing, promoted, for example, by ripples or dunes.

More information about the entrainment process could be inferred, for example, by representing it as a Poisson process or a renewal counting process. However, with one notable exception, sufficient information for our purposes is now at hand. The deficiency concerns the relationship of $\gamma$ to characteristics of the sediment, the flow and the bed. There are semiempirical relationships, such as that of Lane and Kalinske (1939), for predicting the conditions necessary for entrainment. However, none of them are capable of predicting in a probabilistic sense how long a particle will remain on the bed before it is entrained. Hopefully, this will be remedied by future research.

Because of the present lack of rational criteria for selecting appropriate $\gamma$ values, numerical solutions were performed with $\gamma$ ranging all the way from 0.01 to 2,000 . This was done with a view toward demonstrating the effect of the depositionstorage-re-entrainment cycle on the longitudinal dispersion process.

Figure 3-21 shows the effect of $Y$ on the vertical distribution of suspended sediment at $\tau=3$ for the condition that $\beta=0.1$. The results for other combinations of $\tau$ and $\beta$ are qualitatively similar. When the entrainment rate is small $(\gamma=0.02)$, the vertical distribution is nearly the same as for the case of the completely absorbing bed $(\alpha=1)$ with zero entrainment. As $\gamma$ becomes large, the vertical distribution converges to the distribution for the case of the completely reflecting bed $(\alpha=0)$, which corresponds to $\gamma$ approaching infinity.

The results in Figure 3-22, which show the fraction of sediment carried in suspension as a function of dispersion time for $\beta=0.1$, exhibit a similar tendency in that the results for small $\gamma$ approach those for $\alpha=1$ and the results for large $\gamma$ approach those for $\alpha=0$. It is noteworthy that the amount of sediment carried in suspension does not decrease exponentially with time when re-entrainment is occurring. Instead $\mathrm{m}_{0}(\tau)$ tends to approach an equilibrium value as a balance between the deposition rate, $v_{\mathrm{S}} \mathrm{C}_{0}(0+, \tau)$, and the entrainment rate, $\mathrm{\gamma W}_{0}(\tau)$, is achieved. This can be seen from equation 3-11 for $p=0$ together with the condition required by conservation of mass that $m_{0}(\tau)+W_{0}(\tau)=1$.

The remainder of this section is devoted for the most part to the presentation of results obtained with the entrainment factor $\gamma=0.02$ in a flow with mean velocity $\mu=100$, which corresponds to $\bar{U} / U_{\tau}=7$. In general, the results for $\gamma=0.20$ and 2.0 qualitatively resemble those for $\gamma=0.02$, whereas the results for $\gamma \geqslant 20$ tend to resemble more closely those for the reflecting boundary $(\alpha=0)$ case. The results for $\gamma=0.02$ were selected for presentation because they demonstrate more dramatically than the others the importance of even a small amount of reentrainment in the longitudinal dispersion process for suspended particles. The resistance coefficient $\bar{U} / \mathrm{U}_{\tau}$ $=7$ was selected because it is representative of the flow conditions in the Sayre-Chang experiments.

Figure 3-23 shows the mean longitudinal dis placement from the source of all the sediment that is 


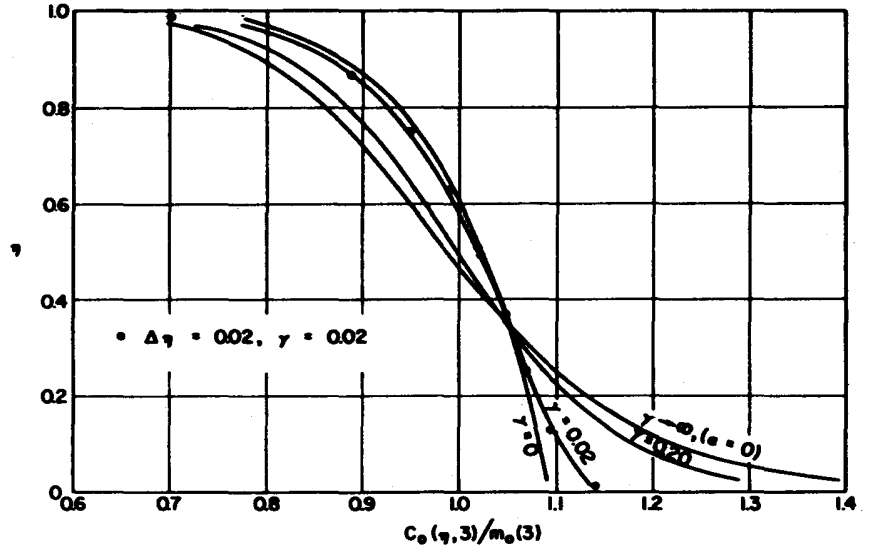

Figure 3-21. Vertical distribution of suspended sediment at $\tau=3$ with entrainment of deposited particles occurring; $\beta=0.1$

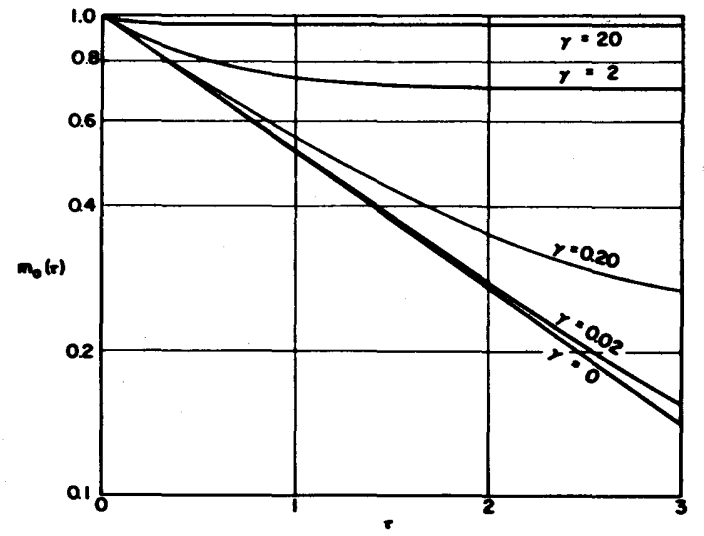

Figure 3-22. Fraction of sedinient carried in suspension as a function of dispersion time with entrainment of deposited particles occurring; $\beta=0.1$

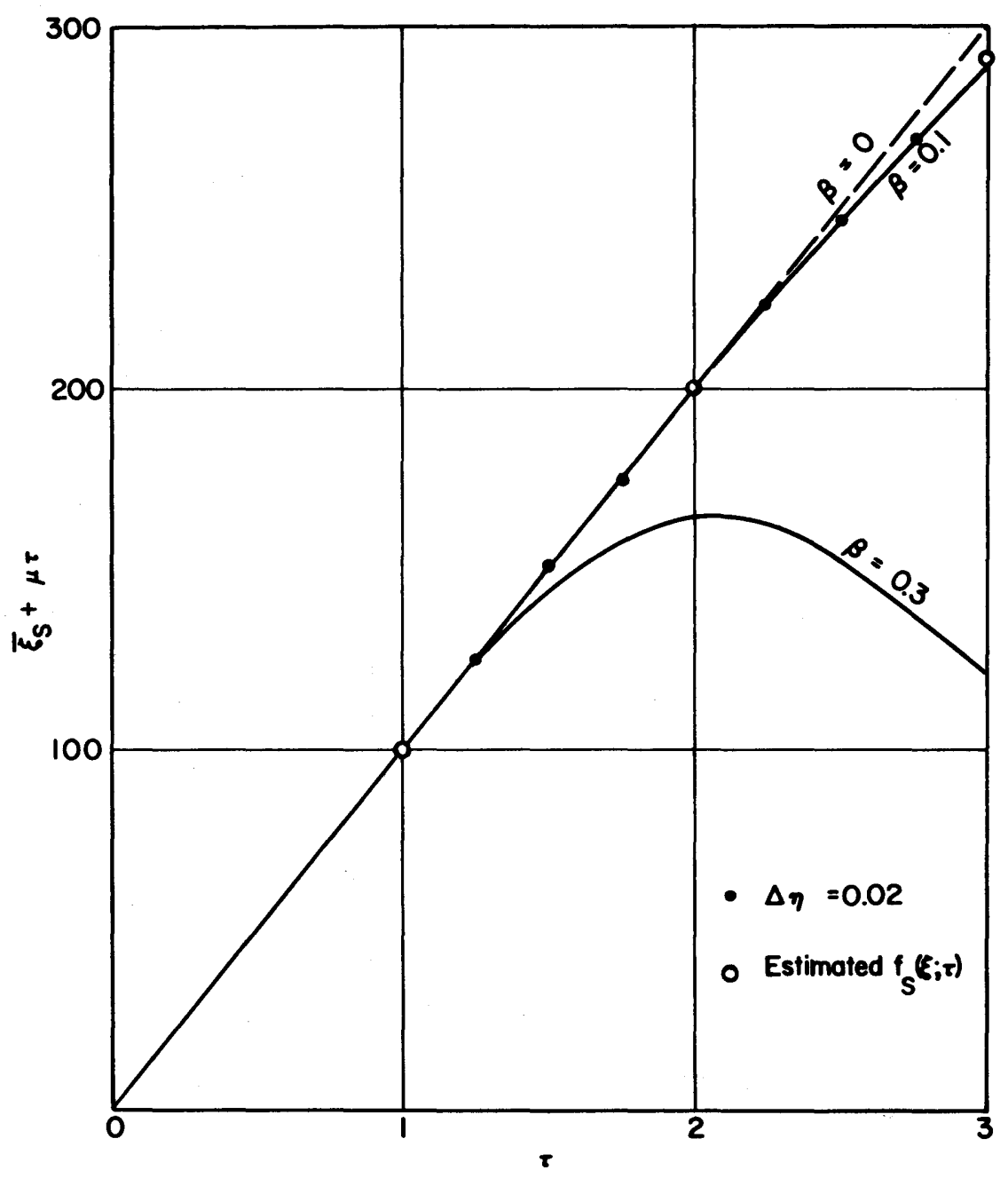

Figure 3-23. Mean longitudinal displacement, from the source, of the suspended sediment as a function of dispersion time in a flow with $\gamma=0.02$ and $\bar{U} / U_{T}=7$ 
in suspension at any time $\tau$. The curious form of the $\beta=0.3$ curve, which seems to show that the sediment is moving backwards when $\tau>2$, is evidently due to the deposition far downstream from the source of sediment which has been continuously transported in suspension, and the re-entrainment further upstream of sediment which has been deposited and temporarily stored on the bed. The reasoning behind this statement will become clearer as a picture of the total process unfolds in the following pages. That such an exchange occurs is demonstrated by the $\mathrm{m}_{0}(\tau)$ results for $\beta=0.3$. They show that the amount of sediment in suspension at $\tau=3$ when $\gamma=0.02$ is about five times greater than when $\gamma=0$. Although these results are not shown here, they are qualitatively similar to the results for $\beta=0.1$ in Figure 3-22 except that the relative difference when $\beta=0.3$ is much larger.

The mean longitudinal displacement results for smaller values of $\beta$ diverge at a much slower rate from the reference displacement for $\beta=0$ indicated by the dashed line in Figure 3-23. This is because the deposition rates, as demonstrated in Figure 3-12, are much less, Consequently, the relative amount of re-entrained sediment in suspension is also much less. However, it is anticipated that at sufficiently large dispersion times the curves representing smaller values of $\beta$ would show the same trend as the curve for $\beta=0.3$.

The variance, $\sigma_{S}^{2}$, of the longitudinal distribution of suspended sediment is shown as a function of $\tau$ in Figure 3-24. The tremendously increased rate of growth of the variance is caused by the reentrainment of deposited particles which add a long tail of suspended sediment to the longitudinal distribution curve. This almost literally becomes a case of the tail wagging the dog.

The results for $\beta=0.1$ and $\gamma>2$ (not shown here) indicate that $\sigma_{S}^{2}$ and $\bar{\xi}_{S}$ vary linearly with $\tau$ after the relative amounts of sediment in suspension and on the bed $m_{0}(\tau)$ and $W_{0}(\tau)$, become constant

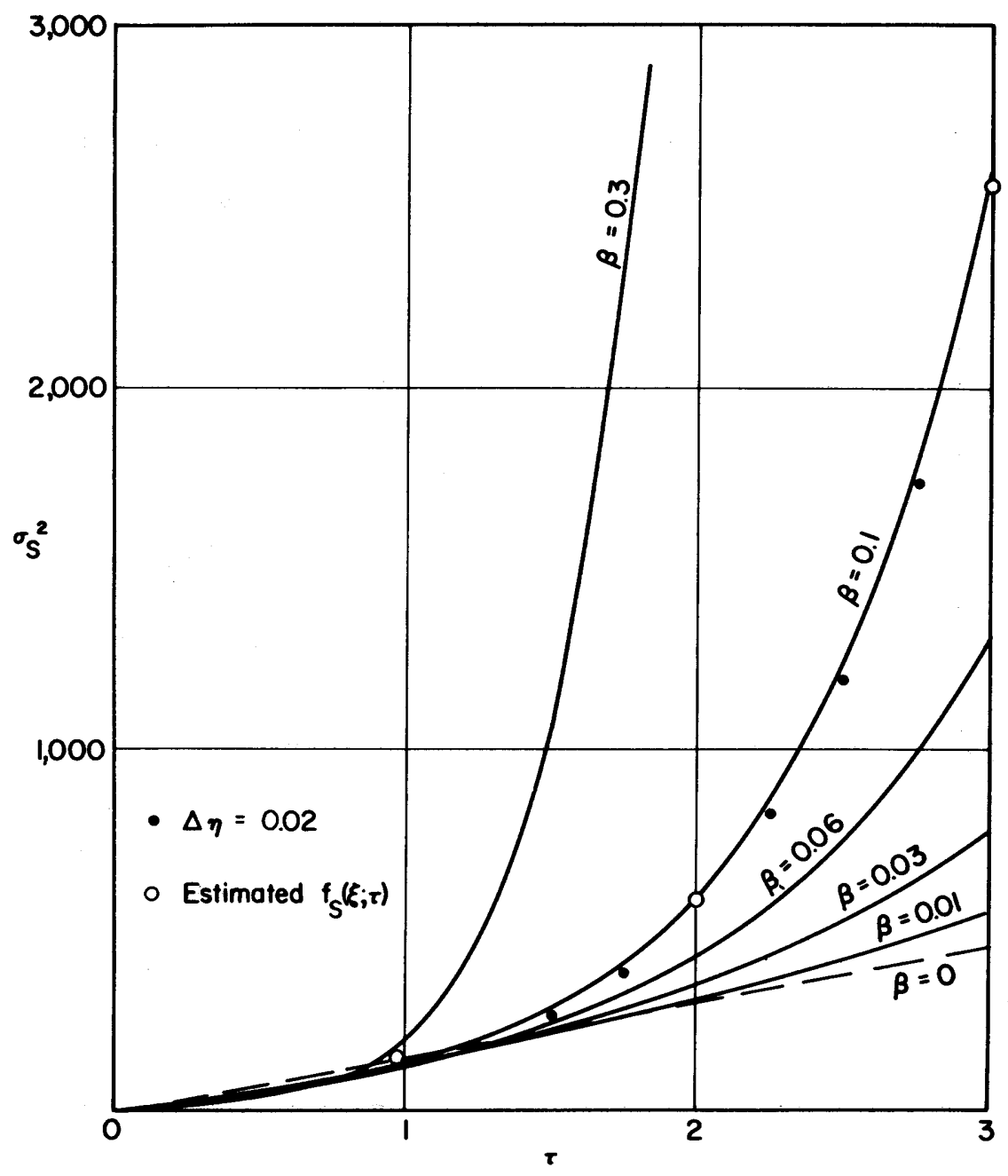

Figure 3-24. Variance of longitudinal distribution of suspended sediment as a function of dispersion time in a flow with $\gamma=0.02$ and $\bar{U} / U_{T}=7$ 
(see Figure 3-22), indicating that overall deposition and re-entrainment rates are in equilibrium. Not until then is there any possibility of representing the longitudinal dispersion of suspended particles as a one-dimensional Fickian diffusion process with a constant dispersion coefficient and mean velocity. Even then $\mathrm{K}_{\xi}$ and $\bar{\mu}_{\mathrm{S}}$ are liable to be complex functions of $\beta, \gamma$ and $\overline{\mathrm{U}} / \mathrm{U}_{\tau}$ in addition to $\mathrm{y}_{\mathrm{n}} \mathrm{U}_{\tau} / \mathrm{D} \kappa^{3}$. It was not possible to ascertain the form of these relationships from the results because the equilibrium condition was not approached in a sufficient number of runs. In only one run $(\beta=0.1, \gamma=2)$ were the results sufficiently different from those for the corresponding runs with $\alpha=0$ to be of any use in this regard. The logic for anticipating that the process must eventually attain the equilibrium stage where it behaves as a onedimensional diffusion process is that, so long as exchange between the bed and the flow is occurring, the bed is in effect just another incremental layer in the system in which the velocity with respect to the moving coordinate system is $-\mu$, and where the exchange coefficient, $\gamma$, at the interface has properties that differ somewhat from the exchange coefficient, $\psi$, in the flow. The essential requirements of Taylor's theory of diffusion by continuous movements, as outlined in Chapter II, Section 3 seem to be satisfied as long as the overall vertical distribution of particles in the system is, if not uniform, at least invariant with respect to time.
Results showing the skew coefficient of the suspended material as a function of $\tau$ are given in Figure 3-25. The general tendency toward large negative skewness is indicative of concentration distribution curves which are heavily weighted at the downstream end with a long tail extending in the upstream direction. The eventual shift from negative to positive skewness in the $\beta=0.3$ curve indicates a shift in weight from the downstream to the upstream end of the concentration-distribution curve. This occurs when the concentration of suspended sediment toward the trailing end of the tail, due to reentrainment from the region of heaviest deposition, overbalances the rapidly diminishing concentrations at the leading end of the curve where deposition greatly exceeds entrainment. Given sufficiently long dispersion time, it is anticipated that the curves representing the smaller values of $\beta$, would behave in a similar manner.

A comparison of the curves in Figures 3-23, 3-24 and 3-25 with the counterpart curves in Figures 3-16, 3-17 and 3-20, for $\alpha=1$ and no reentrainment, shows sharp divergencies. They are all consistent with the trend toward a relative gain in concentration, due to re-entrainment, in the tail toward the upstream end of the concentration distribution curve. The rate at which this process occurs evidently increases with the rate of deposition, which

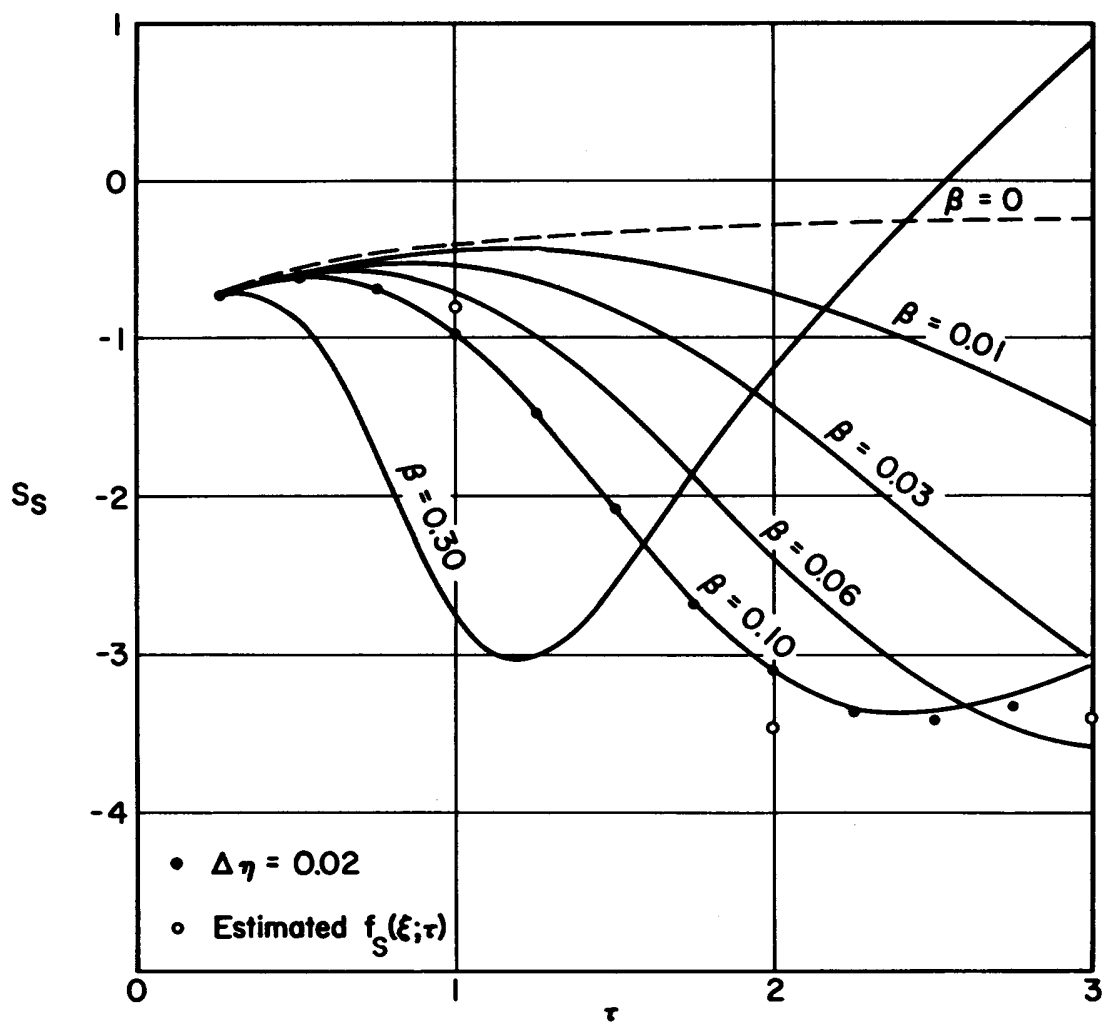

Figure 3-25. Skew coefficient of longitudinal distribution of suspended sediment as a function of dispersion time in a flow with $\gamma=0.02$ and $\bar{U} / U_{\tau}=7$ 
in turn increases with $\beta$. The possibility that this effect may be expressible in a generalized manner, perhaps by incorporating $\beta$ and $\gamma$ into the time scale, although appealing, is beyond the scope of this study.

e. Results for deposited sediment and composite distributions - In the two preceding sections, attention was focused on the suspended particles. In this section, some particular solutions for the deposited sediment and for the combined distribution of the suspended and deposited sediment are examined. As in the last section, the results presented here are restricted to a flow with a logarithmic velocity distribution in which $\kappa=0.42$ and $\bar{U} / U_{T}=7$. Also, the bed is again treated as a completely absorbing boundary.

Figures 3-26, 3-27 and 3-28 show some examples of how the mean displacement, variance and skew coefficient for the sediment deposited on the bed vary with dispersion time for the condition of no reentrainment. Given a sufficiently long time, all of the particles must eventually be deposited on the bed where they will remain at rest. Therefore, all of the parameters describing the distribution of the deposited particles should approach constant asymptotic values. The results for $\beta=0.3$ verify this. Evidently the curves for the other values of $\beta$ require much more time to reach their asymptotic limits.

The fact that the skew coefficients are positive indicates that the distributions are weighted in the upstream direction. This coincides with the intuitive hypothesis that the heaviest deposition should occur close to the source and that the amount of material deposited should decrease with distance going downstream from the source. This line of reasoning suggests that the distribution of deposited particles should follow an exponential function. Using the results for $\beta=0.3$, this hypothesis was tested. It was assumed that with increasing $\tau$ the longitudinal distribution of the deposited particles approaches the exponential probability density function

$$
\mathrm{f}_{\mathrm{W}}(\xi)=\frac{\lambda}{\mu} \mathrm{e}^{-\frac{\lambda}{\mu}(\xi+\mu \tau)} \quad(\xi \geq-\mu \tau) .
$$

The mean displacement, variance and skew coefficient calculated from equation 3-78 are respectively

$$
\begin{aligned}
& \bar{\xi}_{\mathrm{W}}+\mu \tau=\int_{-\mu \tau}^{\infty} \xi \mathrm{f}_{\mathrm{W}}(\xi) \mathrm{d} \xi+\mu \tau=\frac{\mu}{\lambda} \\
& \sigma_{\mathrm{W}}^{2}=\int_{-\mu \tau}^{\infty}\left(\xi-\bar{\xi}_{\mathrm{W}}\right)^{2} \mathrm{f}_{\mathrm{W}}(\xi) \mathrm{d} \xi=\left(\frac{\mu}{\lambda}\right)^{2} \\
& \mathrm{~S}_{\mathrm{W}}=\frac{1}{\sigma_{\mathrm{W}}^{3}} \int_{-\mu \tau}^{\infty}\left(\xi-\bar{\xi}_{\mathrm{W}}\right)^{3} \mathrm{f}_{\mathrm{W}}(\xi) \mathrm{d} \xi=2 .
\end{aligned}
$$

When evaluated for $\mu=100$ and $\lambda=2.29$, which corresponds to $\beta=0.3$ (see Figure 3-12), these equations give the values shown as the asymptotic limits for the $\beta=0.3$ curves shown on Figures 3-26, 3-27 and 3-28. That the curves do indeed approach these values strongly supports the hypothesis that the deposited particles are distributed according to equation 3-78. Additional support is given by the fact that equation 3-78 satisfies equation 3-6 when $y=0$ in the limiting case when

$$
\underset{\tau \rightarrow \infty}{\operatorname{Lim}} \mathrm{C}(\xi, 0+, \tau)=0
$$

At smaller values of $\tau$ when a significant quantity of the sediment is still in suspension, it is reasonable to assume that the distribution of deposited particles can be represented approximately by a truncated exponential distribution function. Given sufficiently large $\tau$, it is expected that the longitudinal distributions for other values of $\beta$ would also converge to equation 3-78.

It is interesting to note that equation 3-78, when expressed for a stationary coordinate system with the origin at the source, has the same form as the probability density function used by Hubbell and Sayre (1964) and Einstein (1937) for the distribution of step lengths in the stochastic model for the longitudinal dispersion of bed-material particles which was mentioned in connection with equation 3-76. This suggests the possibility that $f_{W}(\xi)$ for the initial condition of all particles concentrated near the bed, instead of distributed uniformly with respect to flow depth as with equation 3-78, might turn out to be closely related to the step length distribution function. If this is true, and if $f_{W}(\xi)$ for this initial condition is again an exponential function, it is more than likely that the stochastic dispersion model can be obtained as a particular simultaneous solution of equations $3-2$ and 3-6.

As might be expected, the entrainment factor, $\gamma$, has considerable effect on the distribution of deposited particles. For example, when $\gamma=0.02$, the numerical results show that the displacement of the mean and the variance are slightly larger and the skew coefficient is slightly smaller than when $\gamma=0$. As the entrainment rate increases, the differences become larger. Finally, when the entrainment rate becomes large $(y \geq 20)$, and the residence times of particles on the bed become short, the values of the parameters describing the longitudinal distribution of the particles on the bed approach those of the corresponding parameters describing the distribution of the particles in the bottom increment of the flow for the reflecting boundary case.

Figures 3-29, 3-30 and 3-31 illustrate the variation with respect to dispersion time of the mean displacement, variance and skew coefficient of the composite longitudinal distribution,

$$
f_{T}(\xi ; \tau)=f_{S}(\xi ; \tau)+f_{W}(\xi ; \tau),
$$




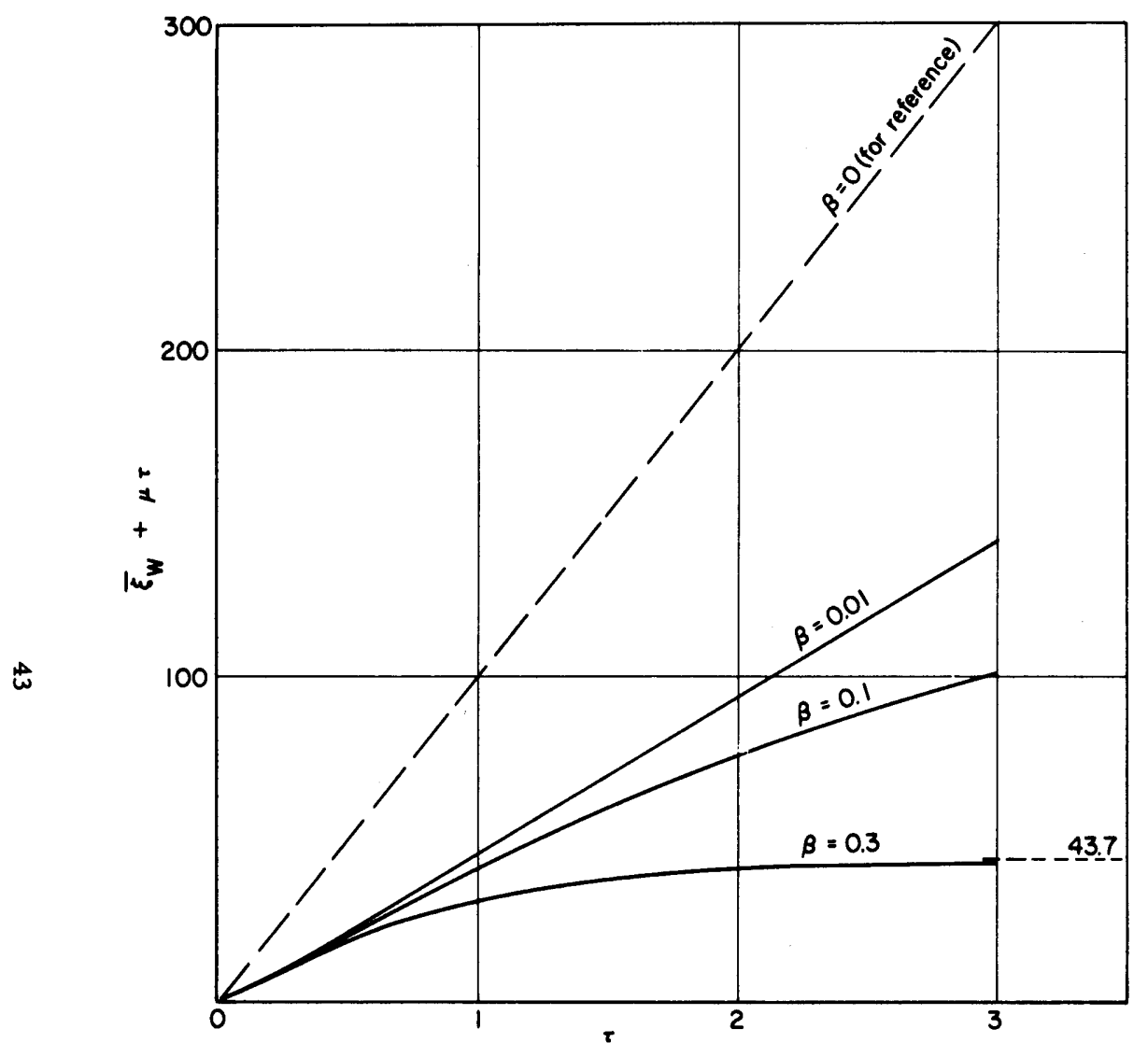

Figure 3-26. Mean longitudinal displacement, from the source, of the deposited sediment as a function of dispersion time in a flow with $\gamma=0$ and $\bar{U} / U_{\tau}=7$

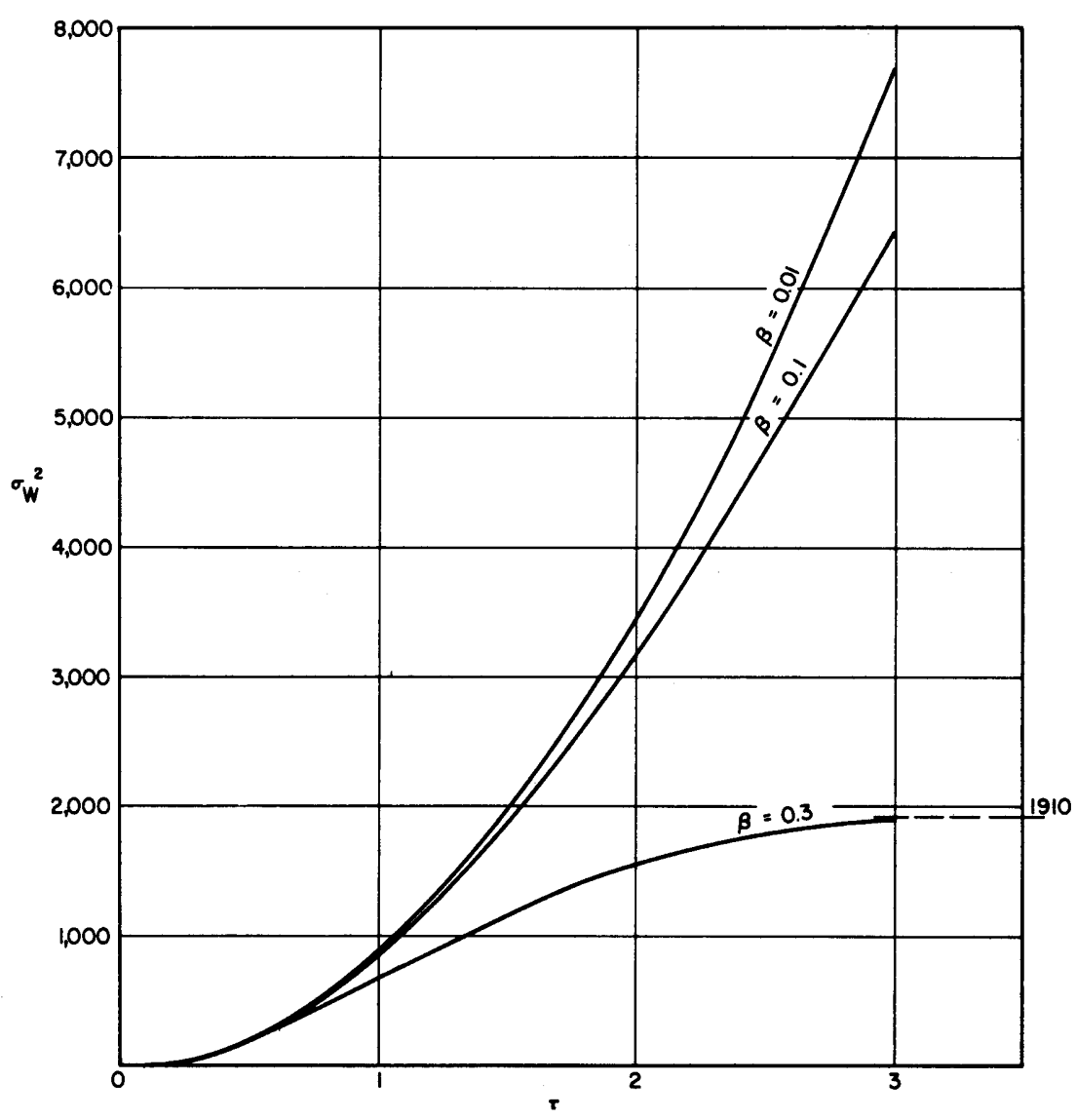

Figure 3-27. Variance of longitudinal distribution of deposited sediment as a function of dispersion time in a flow with $\gamma=0$ and $\bar{U} / U_{\tau}=7$ 


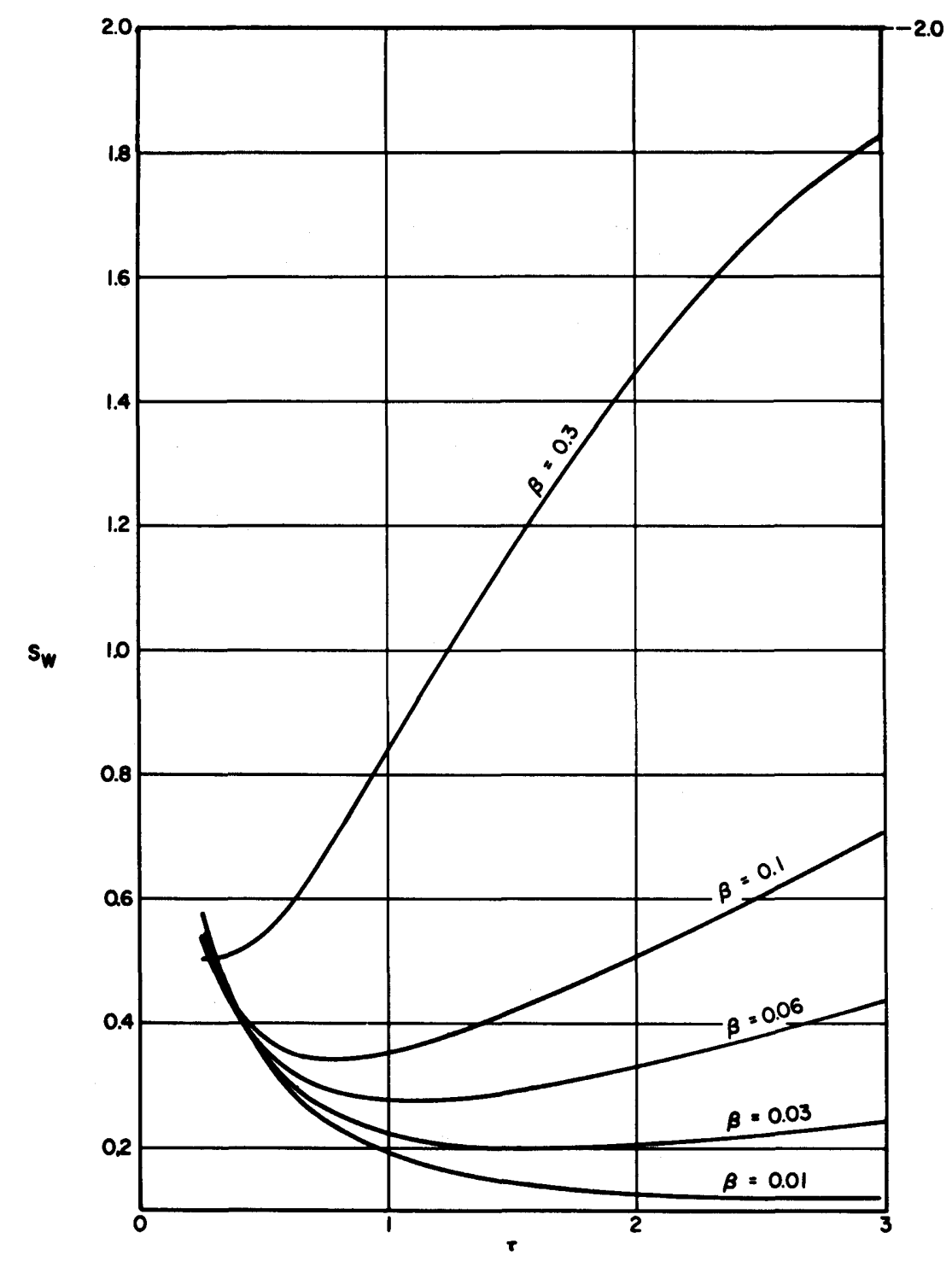

Figure 3-28. Skew coefficient of longitudinal distribution of deposited sediment as a function of dispersion time in a flow with $\gamma=0$ and $\bar{U} / U_{T}=7$

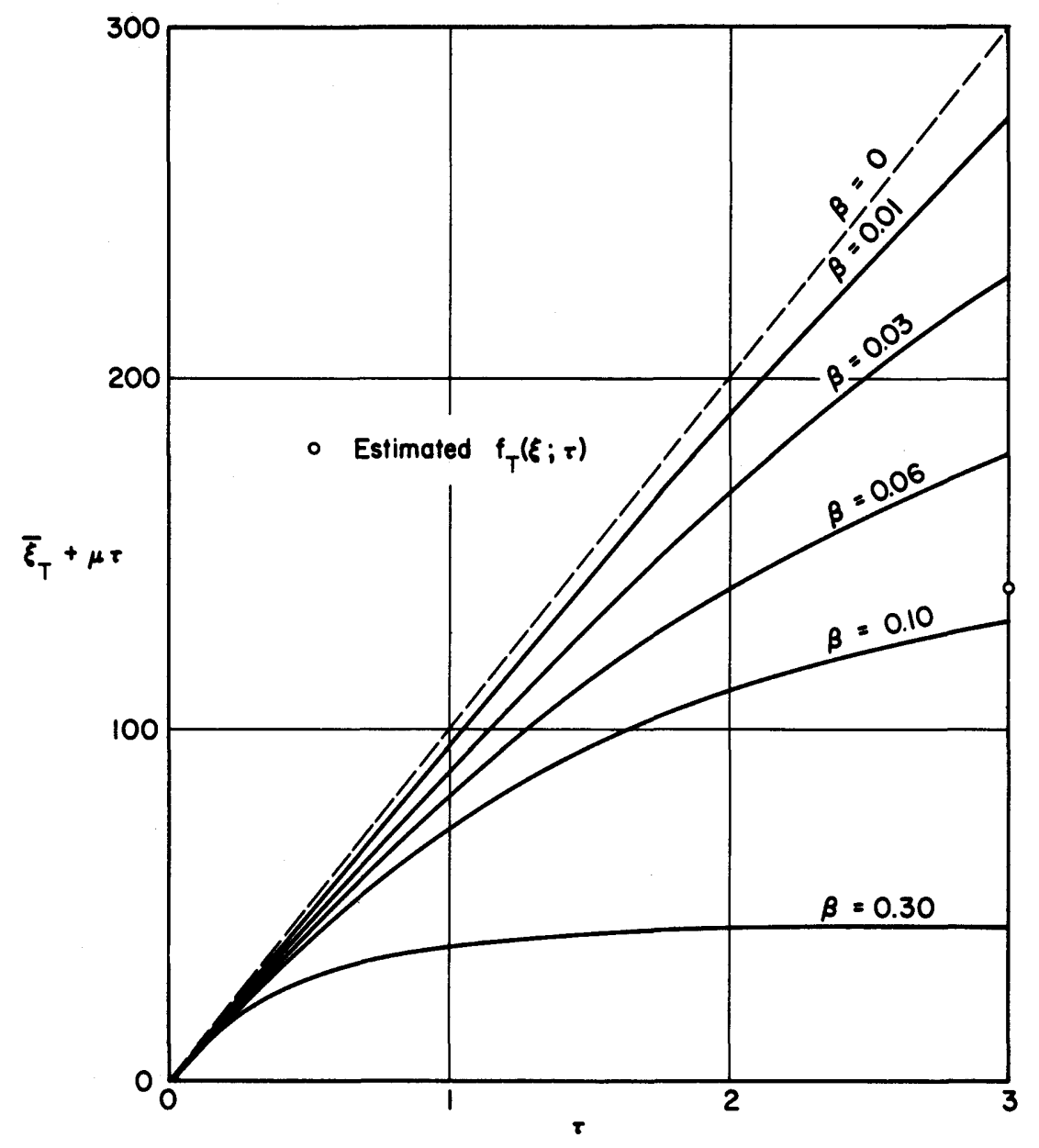

Figure 3-29. Mean longitudinal displacement, from the source, of all the sediment as a function of dispersion time in a flow with $\gamma=0.02$ and $\bar{U} / U_{\tau}=7$ 


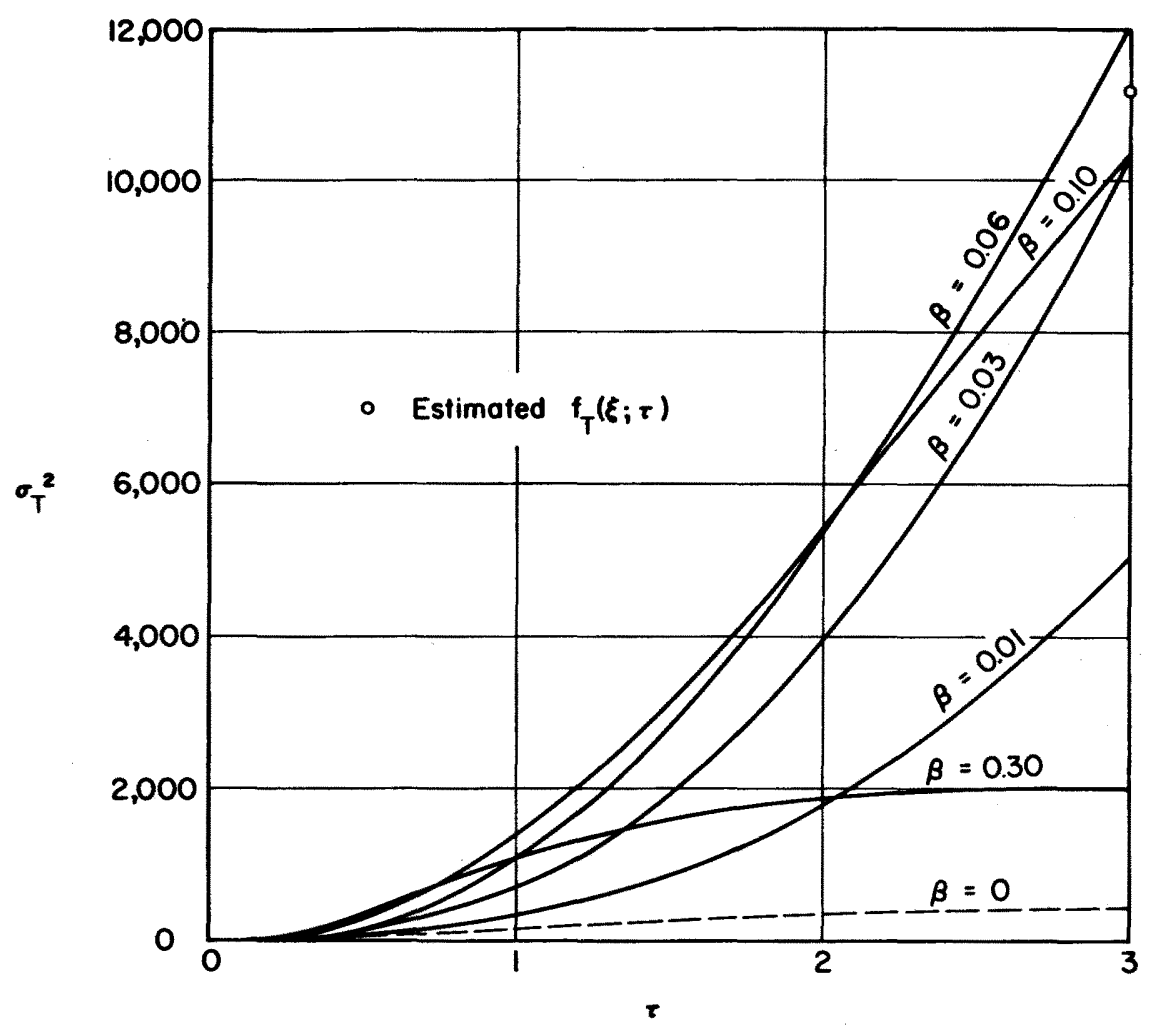

Figure 3-30. Variance of longitudinal distribution of all the sediment as a function of dispersion time in a flow with $y=0.02$ and $\bar{U} / U_{\tau}=7$

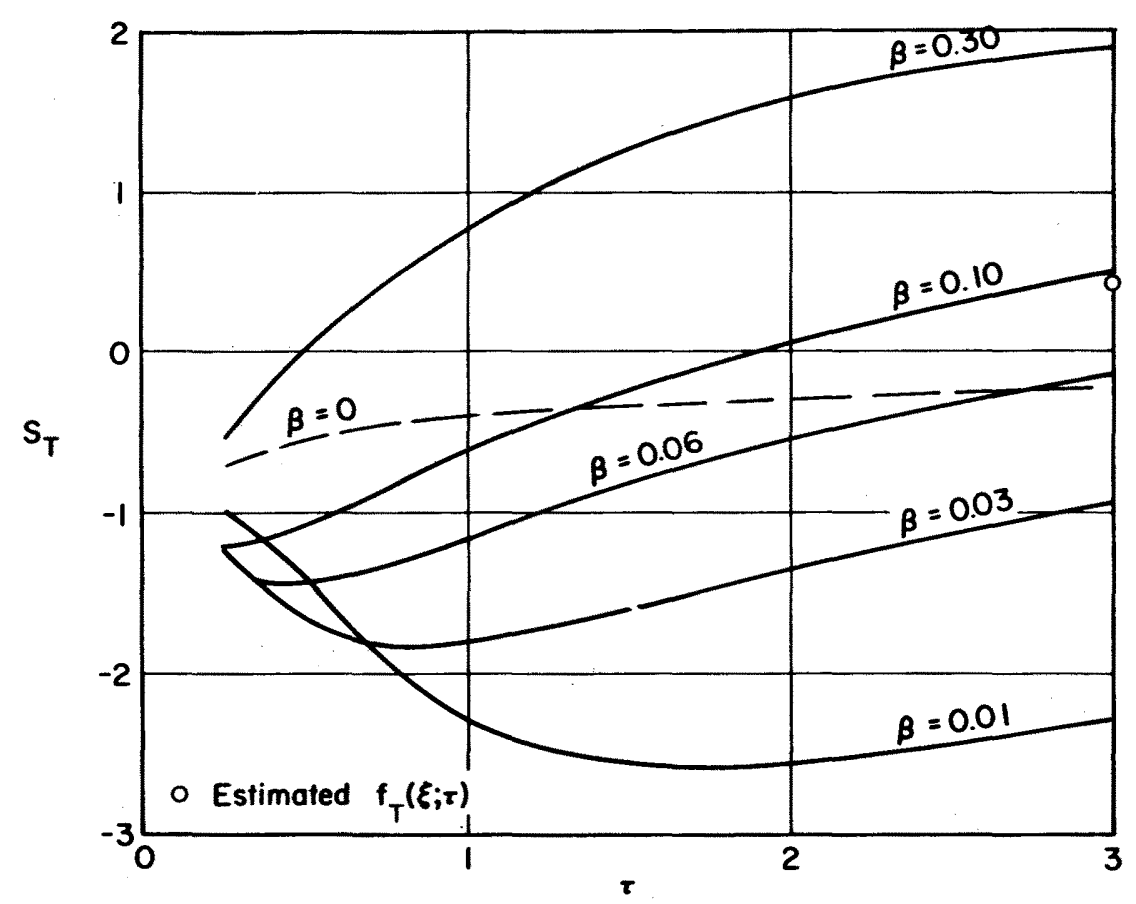

Figure 3-31. Skew coefficient of longitudinal distribution of all the sediment as a function of dispersion time in a flow with $\gamma=0.02$ and $\bar{U} / U_{\tau}=7$ 
of both the suspended and the deposited sediment when $Y=0.02$. Considering the dispersion process as a whole, this is perhaps the most logical form in which to present the results. From an experimental point of view, however, separation into the suspended and deposited components is almost mandatory.

A comparison with the results for the suspended sediment indicates that the results for the composite distribution are more strongly influenced by the tails, which in the composite distribution are composed mostly of deposited material. As with the suspended sediment, here also the results indicate that the mean displacement and variance will not vary linearly with $\tau$ before an equilibrium balance between $\mathrm{m}_{0}(\tau)$ and $\mathrm{W}_{0}(\tau)$ is achieved. Therefore, at low reentrainment rates in particular, a very long dispersion time will be required before the dispersion could possibly be described as a one-dimensional process characterized by a constant mean velocity and dispersion coefficient. The results for the runs with $\gamma=2$ .and $\gamma=20$ for $\beta=0.1$ do indicate that such a relationship exists as an asymptotic limit. As $\gamma$ becomes still larger, the results approach those of the case of the completely reflecting boundary.

f. Estimated longitudinal sediment distribution curves - Throughout the discussion in the preceding two sections it has been implied that the radical departure from the results for $\beta=0$ is due mainly to the influence of tails that are composed of deposited and/or re-entrained particles. In order to test this hypothesis several estimated longitudinal distributions were constructed. The mean displacement, variance and skew coefficients for the estimated distributions were then calculated and compared with results from the numerical solutions. Two examples of the estimated distributions are given in Figures 3-32 and 3-33.

The estimated distribution, $f_{S}(\xi ; \tau)$, for the conditions indicated in Figure 3-32, was obtained by resolving the suspended component into a head and a tail, so that

$$
\mathrm{f}_{\mathrm{S}}(\xi ; \tau)=\mathrm{f}_{\mathrm{h}}(\xi ; \tau)+\mathrm{f}_{\mathrm{t}}(\xi ; \tau)
$$

The function $f_{h}(\xi ; \tau)$ for the head was assumed to be normally distributed with $\mathrm{m}_{0}, \bar{\xi}$ and $\sigma^{2}$ given by the results of the corresponding solution for $\gamma=0$. That is, the head was assumed to be composed entirely of particles which had not been deposited. It was assumed that the tail is composed entirely of reentrained particles so that the area under the tail is given by

$$
\left.\left.m_{0_{t}}=m_{0}\right]_{\gamma=0.02}-m_{0}\right]_{\gamma=0}
$$

Assuming that the truncated exponential form inherited

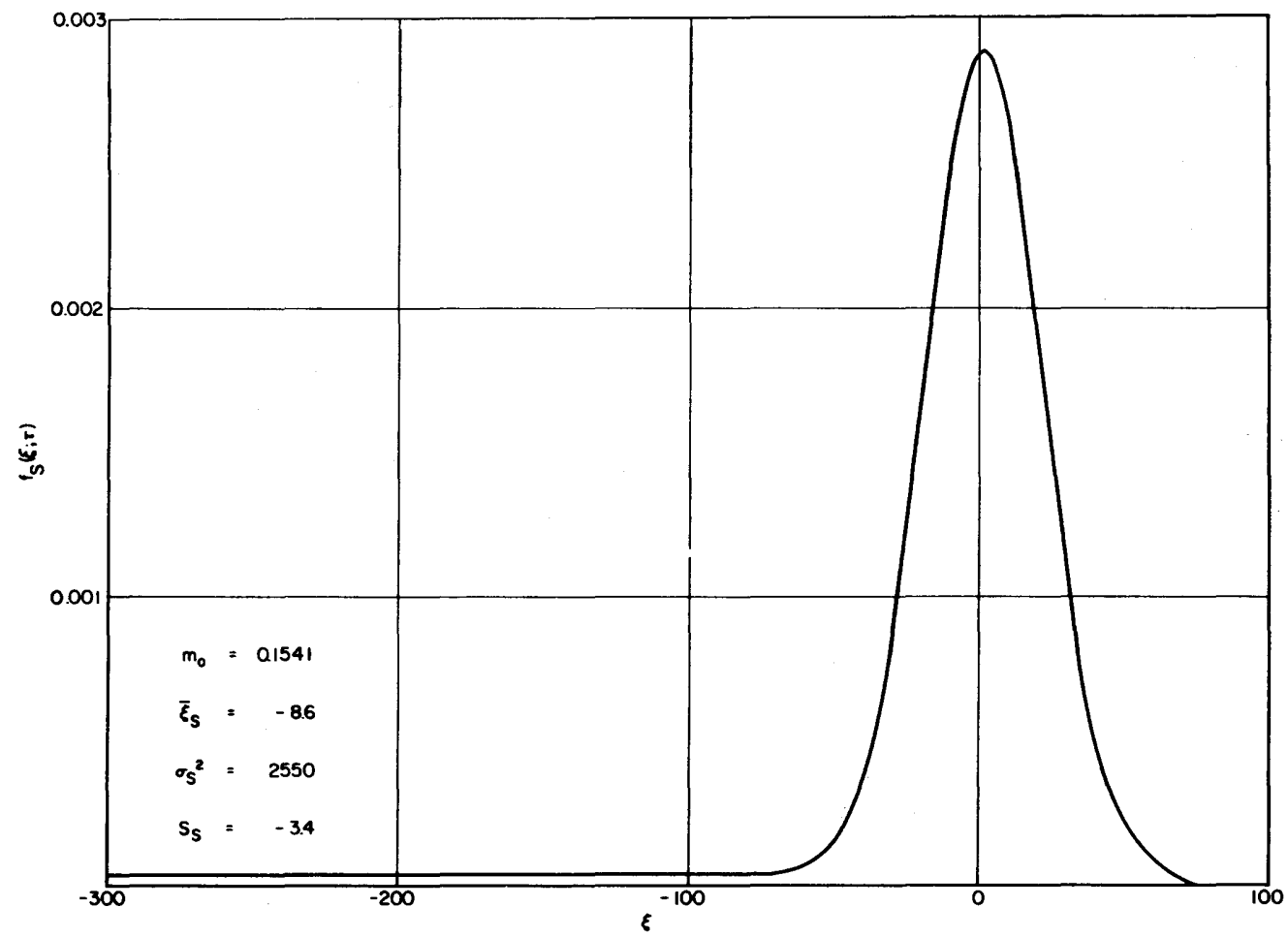

Figure 3-32. Estimated longitudinal distribution of suspended sediment at $\tau=3$ in a flow with $\gamma=0.02, \bar{U} / U_{\tau}=7$, and $\beta=0.1$ 


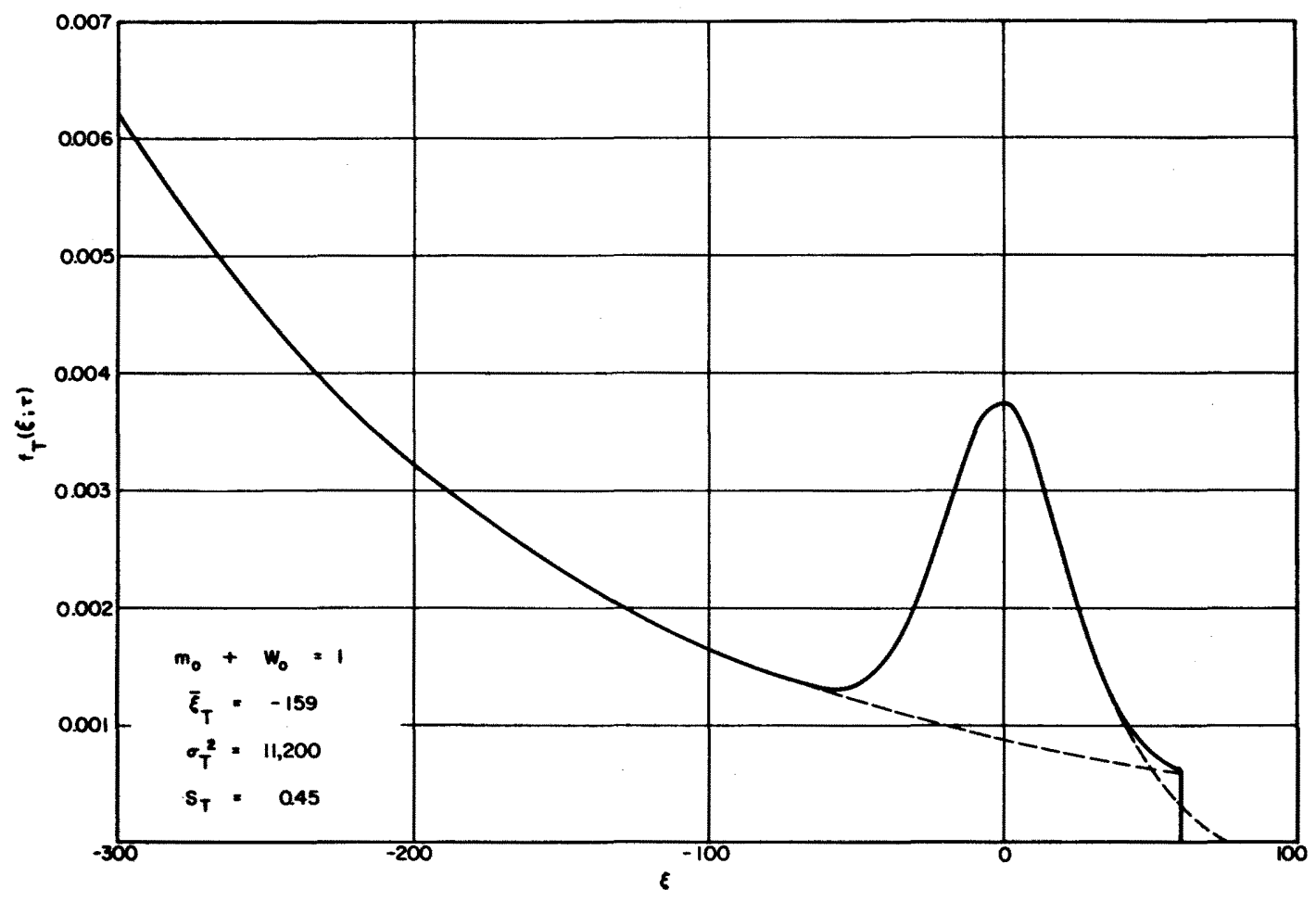

Figure 3-33. Estimated longitudinal composite distribution of all the sediment at $\tau=3$ in a flow with $\gamma=0.02, \bar{U} / U_{\tau}=7$, and $\beta=0.1$

from the deposited particles would tend to be somewhat flattened due to re-entrainment and subsequent transport in suspension, $f_{t}(\xi ; \tau)$ for the tail was represented as a uniform distribution extending from the source at $\xi=-\mu \tau=-300$ to $\xi=3 \sigma=60$. Values of $\bar{\xi}_{\mathrm{S}}, \sigma_{\mathrm{S}}^{2}$ and $\mathrm{S}_{\mathrm{S}}$, calculated from the estimated $f_{S}(\xi ; \tau)$ distributions for $\tau=1,2$ and 3 , are plotted in Figures 3-23, 3-24 and 3-25. The agreement with the curves indicating the numerical solutions is very good, particularly for the mean displacement and variance.

The estimated composite distribution, $f_{T}(\xi ; \tau)$ for both the suspended and deposited sediment at $\tau=3$, which is shown in Figure 3-33, was obtained in much the same manner. Here the head was a assumed to have the same characteristics as in Figture 3-32. However, the tail was represented as an exponential distribution like equation 3-78 with $\mu=100$ and $\lambda=0.655$, but adjusted to give an area under the curve of

$$
\left.m_{0_{t}}=1-m_{0}\right]_{Y=0}
$$

when truncated at $\xi=60$. Values of $\bar{\xi}_{\mathrm{T}}, \sigma_{\mathrm{T}}^{2}$ and $\mathrm{S}_{\mathrm{T}}$, calculated for the estimated $\mathrm{f}_{\mathrm{T}}(\xi ; \tau)$ distribution, are plotted in Figures $3-29,3-30$ and 3-31. The agreement with the curves showing the numerical solutions is again quite good.

Long tails and a rapidly increasing variance, such as seen in the results presented in this section, have also frequently been observed in connection with the longitudinal dispersion of dissolved dispersants in natural streams with irregular banks. This suggests the existence of an exchange mechanism operating between dead zones close to the banks and the main stream analogous to the one postulated in this study for the deposit, storage and re-entrainment of sediment particles. Indeed, Hays, Krenkel and Schnelle (1966) have applied the dead zone concept with considerable success. Fischer (1966) has achieved similar results by taking into consideration the variation of velocity with respect to lateral position in such a channel.

With the exception of some comparisons with experimental data given in the next chaper, this concludes the presentation of results of the numerical solutions. Many interesting results have not been included. This applies particularly to the distributions of $\bar{\xi}_{S}, \sigma_{S}^{2}$ and $S_{S}$ with respect to $\eta$ for the suspended sediment, and to the results for entrainment factors other than $y=0.02$. However, since the computer programs are included in the appendix, it will not be difficult for the interested reader to reproduce these results. 


\section{THE SAYRE-CHANG EXPERIMENTS}

Of the open-channel longitudinal dispersion experiments which have been reported in the literature, those of Sayre and Chang (1966) are best suited for comparison with the results of the numerical solutions presented in Chapter III. This is largely because the theoretical investigation was planned with this purpose in mind. In addition, the Sayre-Chang experiments are among the few in which an approximately twodimensional flow with a logarithmic velocity distribution was verified by measurement. Furthermore, there is no other known source of data on the longitudinal dispersion of suspended sediment in the silt, or coarser, size range. To facilitate the comparison between the theoretical and experimental results, an abbreviated description of the experiments is given here.

\section{Description of Experiments}

All of the experiments were conducted in a $150-\mathrm{ft}$ long recirculating flume with a $7.83-\mathrm{ft}$ wide by $2-\mathrm{ft}$ deep rectangular cross section. Roughness elements, consisting of 3/4-inch high by 3 -inch long wooden cleats, were placed on the bed of the flume in order to induce an approximately two-dimensional flow with a minimum of lateral velocity variation. The arrangement of the roughness elements can be seen in Figure 4-2. Velocity distribution data, averaged across the middle 6 feet of the flume, are shown in Figure 4-1. For comparison, the logarithmic velocity distribution function according to equation 2-28 for $\kappa=0.42$ is also shown. The basic hydraulic data together with the kinds of dispersants used in the experiments are listed in Table 4-1.

The dispersant was dumped into the flume from an elevated trough which extended across the flume a short distance above the water surface in such a way that the initial distribution of dispersant approximated an instantaneous plane source, uniformly distributed over the entire flow cross section. The concentration of dispersant was then measured as a function of time at each of four downstream sampling stations as the slug of dispersing material passed by. The dispersion distances, defined as the distances from the source to the sampling probes, were 32.8 , $65.6,88.5$, and $115.0 \mathrm{ft}$. The source was always located at least $30 \mathrm{ft}$ downstream from the flume entrance, which was far enough so that a fully developed turbulent flow should have existed over most, if not all, of the test reach.

Two sampling and concentration measuring systems, one continuous and the other discrete, were used in the experiments. Both systems are shown in Figure 4-2. In the continuous system, used mainly

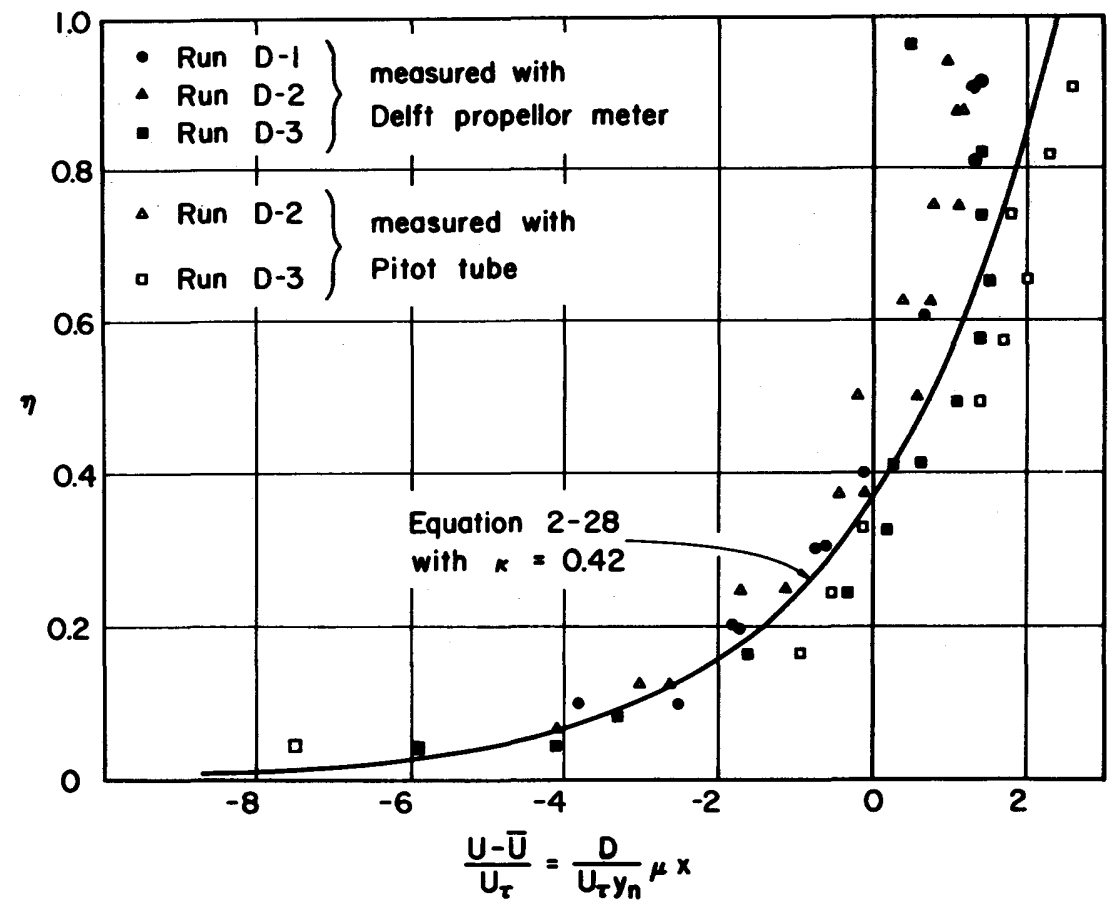

Figure 4-1. Velocity distribution data from dispersion experiments 
TABLE 4-1. HYDRAULIC CONDITIONS IN DISPERSION EXPERIMENTS

\begin{tabular}{|c|c|c|c|c|c|c|c|}
\hline Run & $\begin{array}{c}\mathrm{Q} \\
\mathrm{cfs}\end{array}$ & $\mathrm{ft}_{\mathrm{ft}}$ & $\begin{array}{c}\overline{\mathrm{U}} \\
\mathrm{ft} / \mathrm{sec}\end{array}$ & $\mathrm{S}_{\mathrm{e}}$ & $\begin{array}{c}\text { Temperature } \\
{ }^{0} \mathrm{C}\end{array}$ & Dis pers ant & $\beta$ \\
\hline$D-1$ & 2.88 & 0.493 & 0.747 & 0.001 & 20 & Rhodamine-B dye & 0 \\
\hline D-2 & 7.17 & 0.798 & 1.15 & 1 & 20 & Rhodamine-B dye & 0 \\
\hline$D-3$ & 14.9 & 1.217 & 1. 56 & & 5 & Pontacyl brilliant pink dye & 0 \\
\hline FS - 1 & 2.88 & 0.493 & 0.747 & & 20 & $15-30 \mu$ natural silt & 0.013 \\
\hline FS - 2 & 7.17 & 0.798 & 1. 15 & & 20 & $15-30 \mu$ natural silt & 0.010 \\
\hline $\mathrm{CS}-1$ & 2.88 & 0.493 & 0.747 & & 20 & $53-62 \mu$ natural silt & 0.13 \\
\hline FG-1 & 2.93 & 0.486 & 0.770 & & 7 & $<44 \mu$ glass beads & 0.031 \\
\hline FG-3 & 14.9 & 1.217 & 1. 56 & & 6 & $<44 \mu$ glass beads & 0.019 \\
\hline $\mathrm{CG}-1$ & 2.93 & 0.486 & 0.770 & & 5 & $53-62 \mu$ glass beads & 0.090 \\
\hline $\mathrm{CG}-2$ & 7.12 & 0.814 & 1.12 & & 4 & $53-62 \mu$ glass beads & 0.066 \\
\hline $\mathrm{CG}-3$ & 14.9 & 1.217 & 1.56 & & 6 & $53-62 \mu$ glass beads & 0.058 \\
\hline
\end{tabular}

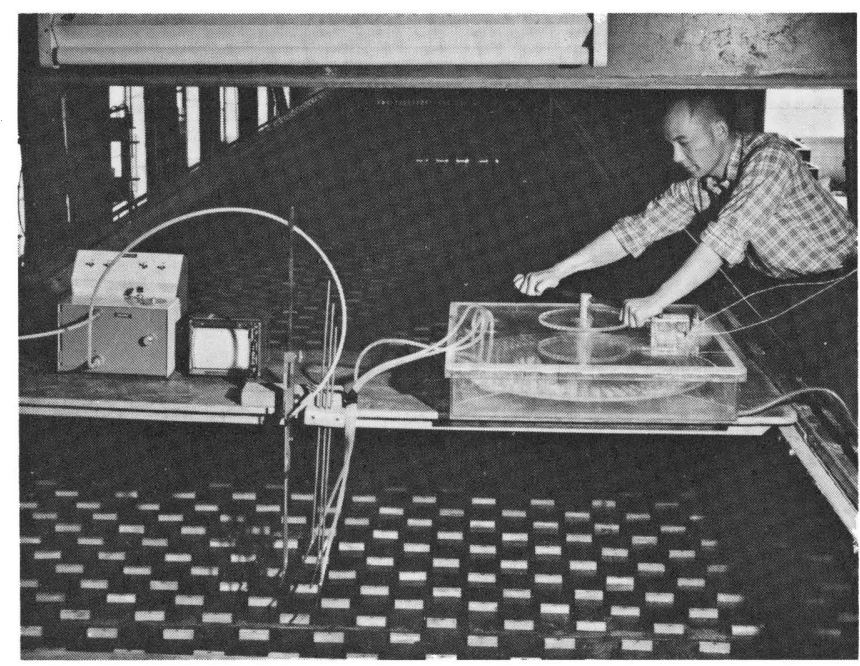

Figure 4-2. Continuous and discrete sampling systems used for determining dye and sediment concentrations in flume dispersion experiments 
for the dye dispersion experiments, the sample passed through a 1/4-inch diameter probe, positioned at $\eta=0.5$, and on through a length of polyethylene tubing to a Model 111 Turner Fluorometer which gave a continuous read out, proportional to concentration, on a strip-chart recorder. Because there was only one fluorometer, the dumping and concentration measuring operation had to be repeated at each of the four dispersion distances. In the discrete system, used in the silt dispersion experiments, the samples passed through 1/4-inch I. D. probes, positioned at $\eta=0.25,0.50$ and 0.75 , and on through mayon tubes to an enclosed box where three sets of vials, one for each sampling depth, were filled simultaneously in rapid sequence. The concentration of dispersant in each vial was determined later with the fluorometer. The sampling box was equipped with a microswitch, wired to an event recorder so that a record of the sampling time for each vial was obtained. There were four complete sets of discrete sampling equipment, so that for a given run, only one dumping was required.

In the silt dispersion experiments, the continuous system was set up at one of the four sampling locations so that a direct comparison of the performance of the two systems could be made. In both systems there was a certain amount of lag and distortion due to dispersion in the sampling tubes, and in the continuous system to the response characteristics of the fluorometer. Response functions for both systems were determined, under simulated experimental conditions, and used to correct the times and the variances of the observed concentration versus time curves. In their report, Sayre and Chang (1966) gave a complete description of the analysis, procedure and experiments involved in making these corrections. They concluded that the lag and distortion, although significant, did not have a major effect on the experimental results.

When used for determining silt concentrations, the fluorometer was converted to a nephelometer, by installing the appropriate combination of light sources and filters. A nephelometer is an instrument which measures concentrations of dilute suspensions from the amount of scattered light. Some examples of longitudinal dispersion data obtained in this manner with the discrete sampling system are shown in Figure 4-3.

Fall-velocity distribution curves for the particles used in the silt-dispersion experiments are shown in Figure 4-4. In computing the values of $\beta=V_{S} / \kappa U_{\tau}$ listed in Table 4-1, median fall velocities, corrected for temperature by Stokes law, were used.

\section{Comparison of Numerical Solutions With Experi- mental Results}

Unfortunately, the results of the numerical solutions can be compared to the experimental results only in a manner which is somewhat indirect. This is because solutions of the Aris moment equations are for instantaneous spatial distributions of dispersant, whereas the experimental results are based on observations of concentrations obtained at a point which are varying in the time domain. The dichotomy seems to be unavoidable because on the one hand the Aris moment transformations in the time domain are not readily applicable, and on the other hand it is not as a rule feasible to experimentally determine the instantaneous distribution of dispersant in the space domain. Even though the properties of the longitudinal dispersion process as viewed in the two domains are like two sides of the same coin, results based on the moments of the distribution curves are not in general transferable from one domain to the other except through the use of approximations. The approximations most commonly employed are based on Taylor's hypothesis,

$$
\frac{\partial}{\partial t} \approx-\bar{U} \frac{\partial}{\partial x},
$$

which is equivalent to stating that no appreciable deformation of the slug of dispersing material occurs during the time required for the slug to pass the sampling probe. From this relationship, approximations such as

$$
\left.\begin{array}{rl}
\mathrm{t} & \approx \mathrm{x} / \overline{\mathrm{U}} \\
\overline{\mathrm{t}} & \approx \overline{\mathrm{x}} / \overline{\mathrm{U}} \\
\sigma_{\mathrm{t}}^{2} & \approx \sigma_{\mathrm{x}}^{2} / \overline{\mathrm{U}}^{2}
\end{array}\right\}
$$

are obtained. Provided that the rate of convection is large in comparison to the rate of spread, i. e., that

$$
\overline{\mathrm{U}}>>\frac{\mathrm{d} \sigma \mathrm{x}}{\mathrm{dt}},
$$

the error involved in these applications of Taylor's hypothesis is small. Where this is not the case, for example, when there is a combination of deposition and re-entrainment, considerable error may be involved.

As the longitudinal dispersion progresses to the stage where it behaves as a one-dimensional process, it has been shown by Fischer (1966) that the relationships

$$
\bar{U}=\frac{d \bar{x}}{d t}=\left(\frac{d \bar{t}}{d x}\right)^{-1}
$$

and

$$
K_{x}=\frac{1}{2} \frac{d \sigma_{x}^{2}}{d t}=\frac{\bar{U}^{3}}{2} \frac{d \sigma_{t}^{2}}{d x}
$$

become identically true, Equations 4-2 and 4-3 provide a solid, if somewhat limited, basis for comparing results obtained in the space and time domains.

a. Dispersion of fluorescent dye - The results of the numerical solution for the case where $\beta=0$ are best compared to the results of the dye dispersion experiments through the use of equation 3-56, the Pearson Type III distribution function. In order to construct a concentration versus time curve in this manner from the results of the numerical solution, $t$, 

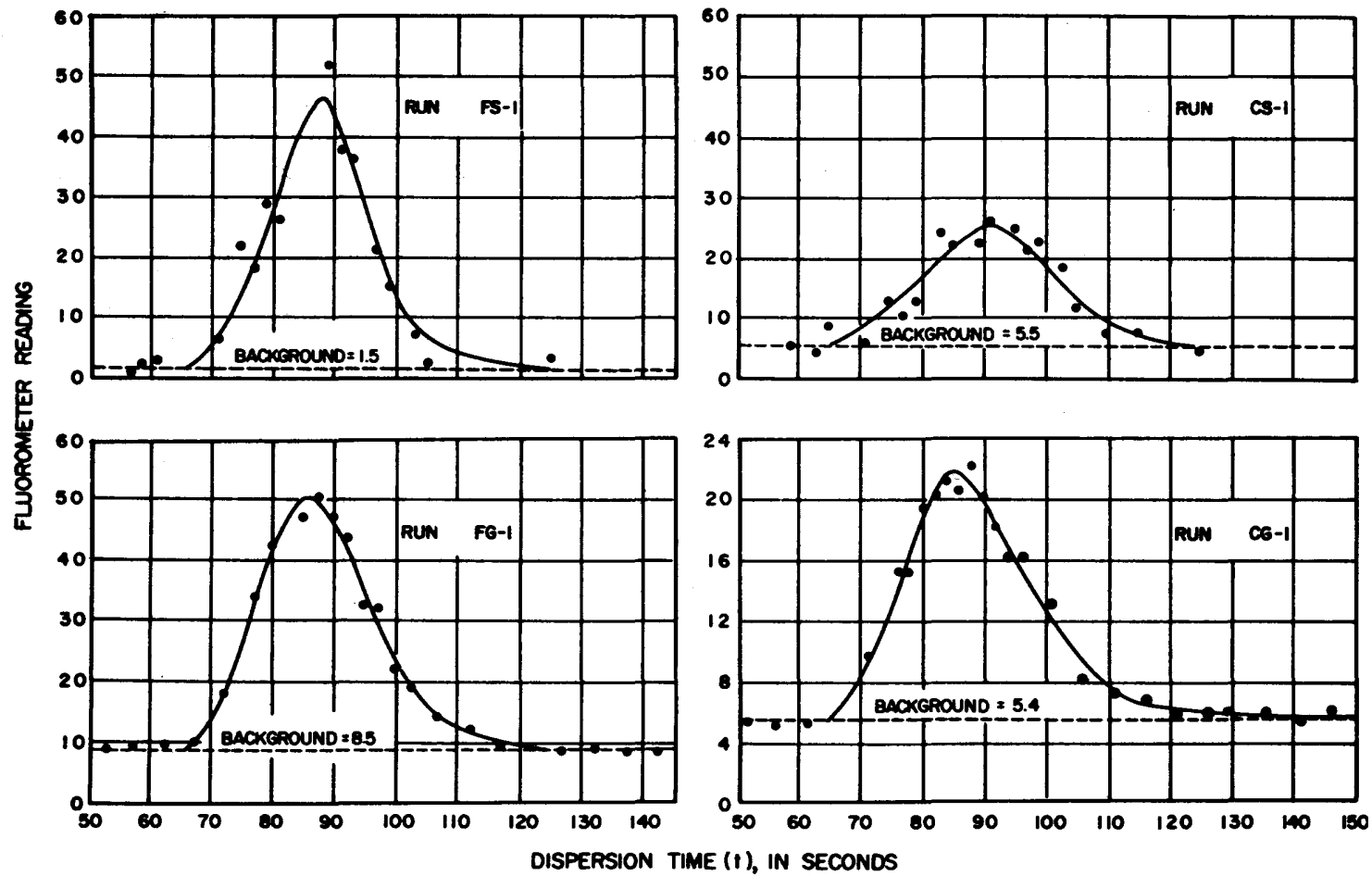

Figure 4-3. Typical longitudional dispersion data for natural silt and glass beads, flow $1, x=65.6 \mathrm{ft}, \eta=0.50$

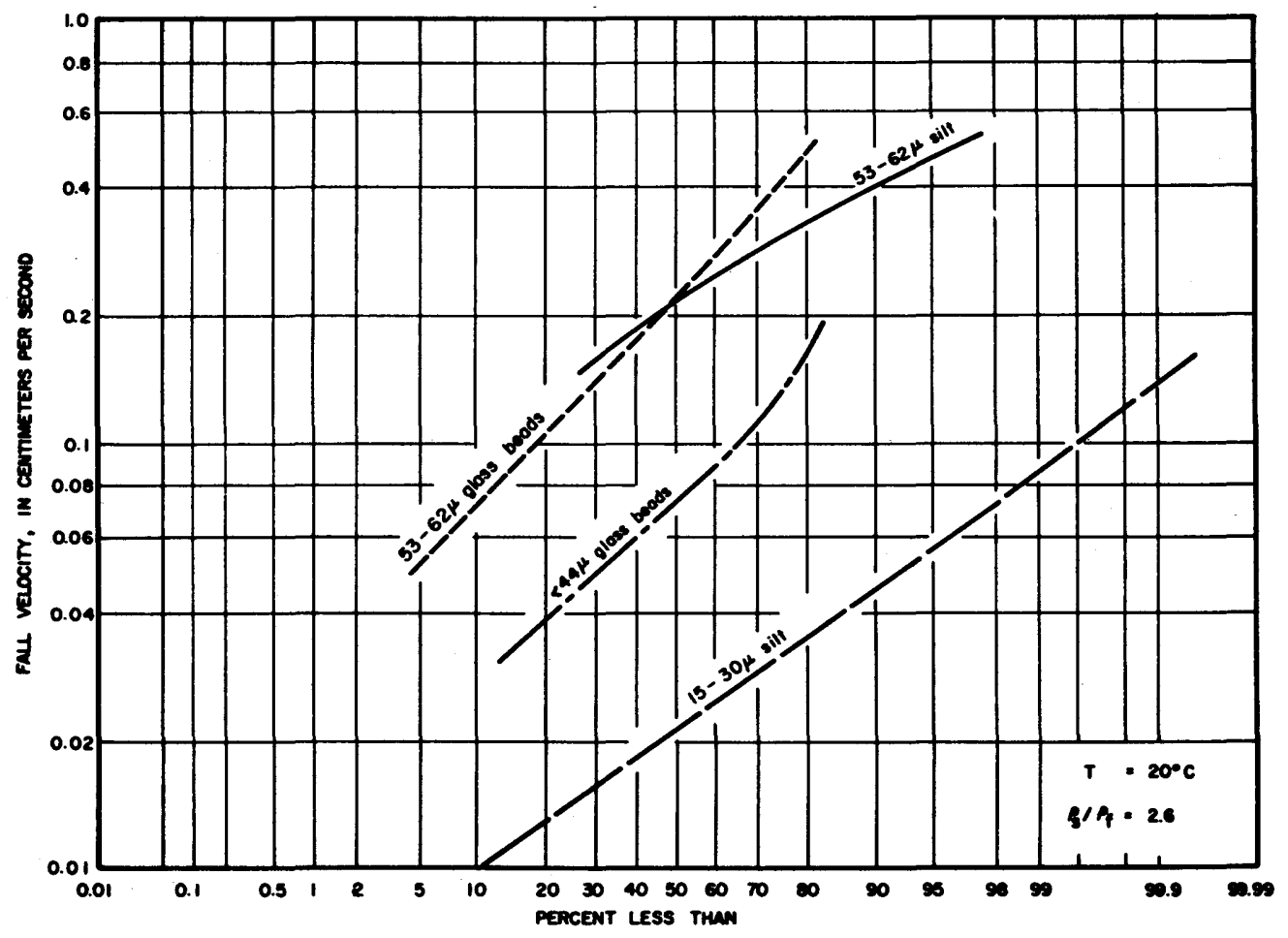

Figure 4-4. Fall velocity distribution curves for the sediments used in the experiments 
in the parameter $\xi=(x-\bar{U} t) / y_{n}$, is varied to corres pond to each point on the curve whereas $x$ is held constant. Also, the parameters $m, b$ and a which are functions of $\tau$, and therefore of $t$, must be recomputed for each point on the curve. In this manner, and changing back to dimensional units according to equations 2-22, we obtain

$$
f(t ; x, \eta)=\frac{\bar{U}}{y_{n}} f(\xi ; m, b, a) .
$$

Because the experimental data were obtained at the sampling depth $\eta=0.50$, the parameters $\mathrm{m}, \mathrm{b}$ and a were computed from the relationships

$$
\left.\begin{array}{l}
\bar{\xi}=0.349 \\
\sigma_{\xi}^{2}=\frac{1}{\kappa^{4}}[4.85 \tau-0.54] \\
S=-0.418(\tau+0.07)^{-1 / 2},
\end{array}\right\}
$$

together with equations 3-57 instead of from equations 3-58. Equations 4-5 are empirical approximations of the results of the numerical solution for $\eta=0.50$.

Equation 4-4 together with its parameters was solved with an IBM 1620 computer for values of $\overline{\mathrm{U}}$, $y_{n}, x$ and $\kappa$ corresponding to those in the experiments. The computer program, in Fortran II, is given in the appendix. The results are shown in Figures 4-5, 4-6 and 4-7, where they are compared with the results of the experiments. The experimental concentration distribution curves were normalized according to the relationship

$$
f(t ; x, \eta)=\frac{C(t, x, \eta)}{\int_{0}^{\infty} C(t, x, \eta) d t}
$$

to obtain the relative concentrations. The agreement between the two sets of curves is quite good. This is underlined by the fact that experimental curves when repeated, tend to differ as much between themselves as from the curves obtained from the numerical solutions.

It should be noted that in the figures the time scales for the experimental curves were shifted respectively $3.1,1.9$ and 3.4 seconds for Runs D-1, D-2 and D-3. These shifts represented the average amounts by which the mean times indicated by the numerical solutions exceeded those indicated by the experiments. The probable explanation is that in the experiments the initial distribution of dye tended to be weighted somewhat toward the water surface, so that the dye tended to move slightly faster than the mean flow velocity at the beginning of the dispersion process. It is also possible that the time-lag corrections indicated by the system response experiments, which were respectively 6. 4, 6. 4 and 5.1 seconds for Runs D-1, D-2 and D-3, were somewhat excessive.
In Figure 4-8, the mean times, variances and skew coefficients computed from the approximate theoretical curves in Figures 4-5, 4-6 and 4-7 are compared with those computed from the experimental data. Except for a displacement which does not seem to vary appreciably with $x$, there is good agreement in the $\bar{t}$ and $\sigma_{t}^{2}$ relationships. In the skew coefficient relationship there is both scatter and displacement. The scatter is no doubt mostly traceable to the tails of the experimental curves, to which the skew coefficients are extremely sensitive, where concentration measurements are the least accurate. The displacement in all of the relationships is consistent in direction, if not in magnitude, with the hypothesis that the initial distribution of dye tended to be weighted toward the water surface. If this is correct, then the dye would not have been subjected to the full dispersive capacity of the flow during the earliest stage of the process.

The dashed lines drawn on the variance relationship represent the Taylor's hypothesis approximation

$$
\sigma_{t}^{2} \approx \sigma_{x}^{2} / \bar{U}^{2}
$$

The displacement of the dashed lines from the solid ones give some idea of the amount of error introduced by this approximation.

b. Dispersion of suspended silt - A comparison of the experimental and theoretical mean rates at which particles settle out of suspension is shown in Figure 4-9. The $\beta$ values for the data points are based on median terminal fall velocities which were determined in still water. The hydrometer method was used for the glass beads, the pipette method for fine natural silt, and the visual accumulation tube method for the coarse natural silt. The $\lambda$ values for the data points are based on experimental observations of the rate of decrease in the amount of sediment remaining in suspension under conditions of open-channel turbulent flow. The curve represents the results of the numerical solution. The agreement between the data points and the curve is reasonably good except for the point farthest to the right which represents Run CS-1. Comparisons such as that in Figure 4-9 could be useful for determining whether the time-averaged settling velocity of sediment particles in open-channel turbulent flow is actually equal to the terminal fall velocity under quiescent conditions.

In the remaining portion of this section, the comparison between results of the numerical solution and the silt dispersion experiments is restricted to the experiments with particles in the $53-62 \mu$ size range. This is because the experimental results obtained in runs with the finer particles, where $\beta \leq 0.031$, except for the tendency of the particles to settle and deposit, differed from the results of the dye dispersion experiments by an amount which was within the limits of experimental error. This is evidently because not enough sediment was ever deposited 


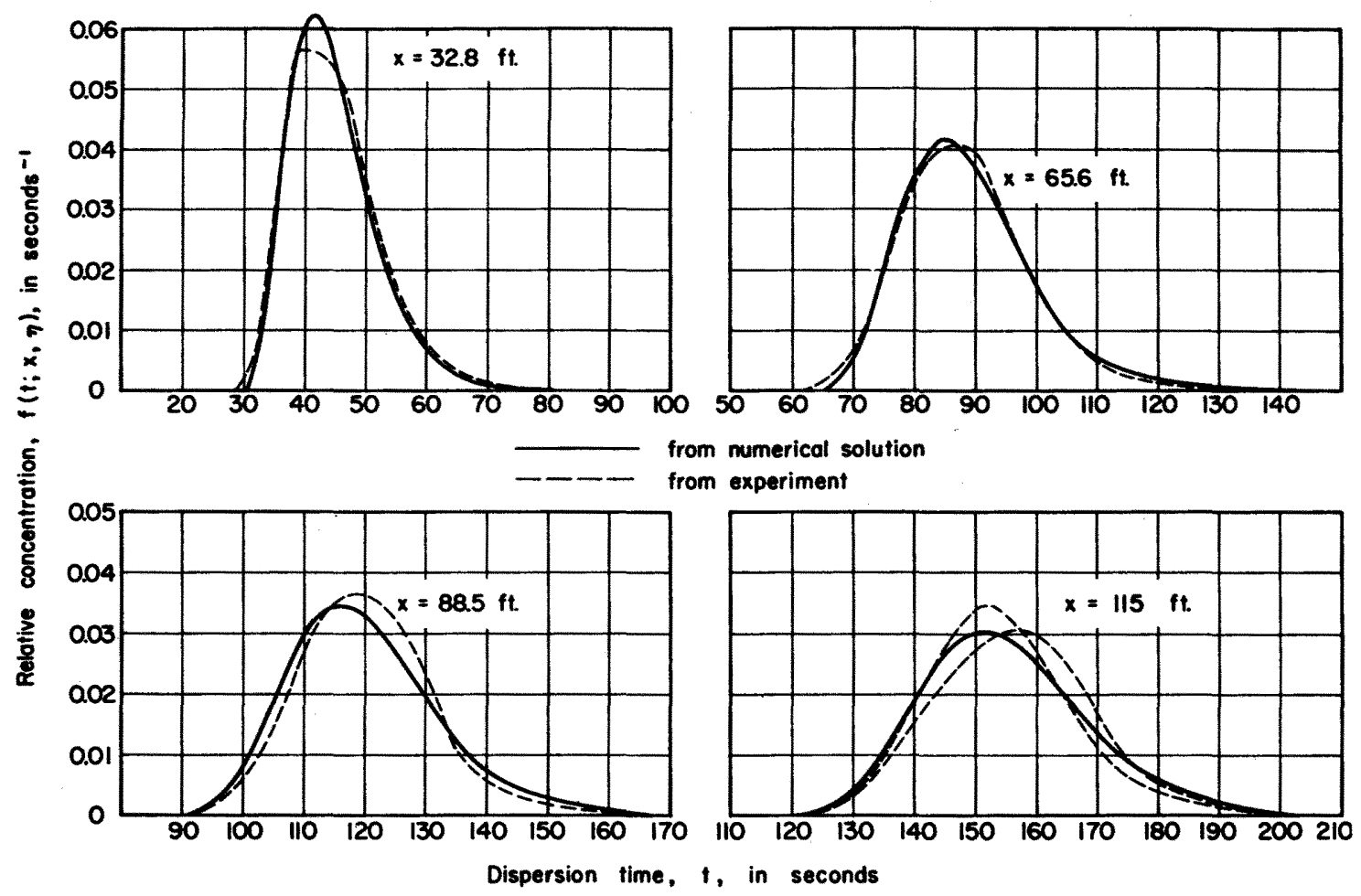

Figure 4-5. Comparison of experimental and approximate theoretical relative concentration versus time curves, longitudinal dispersion of dye, Run D-1

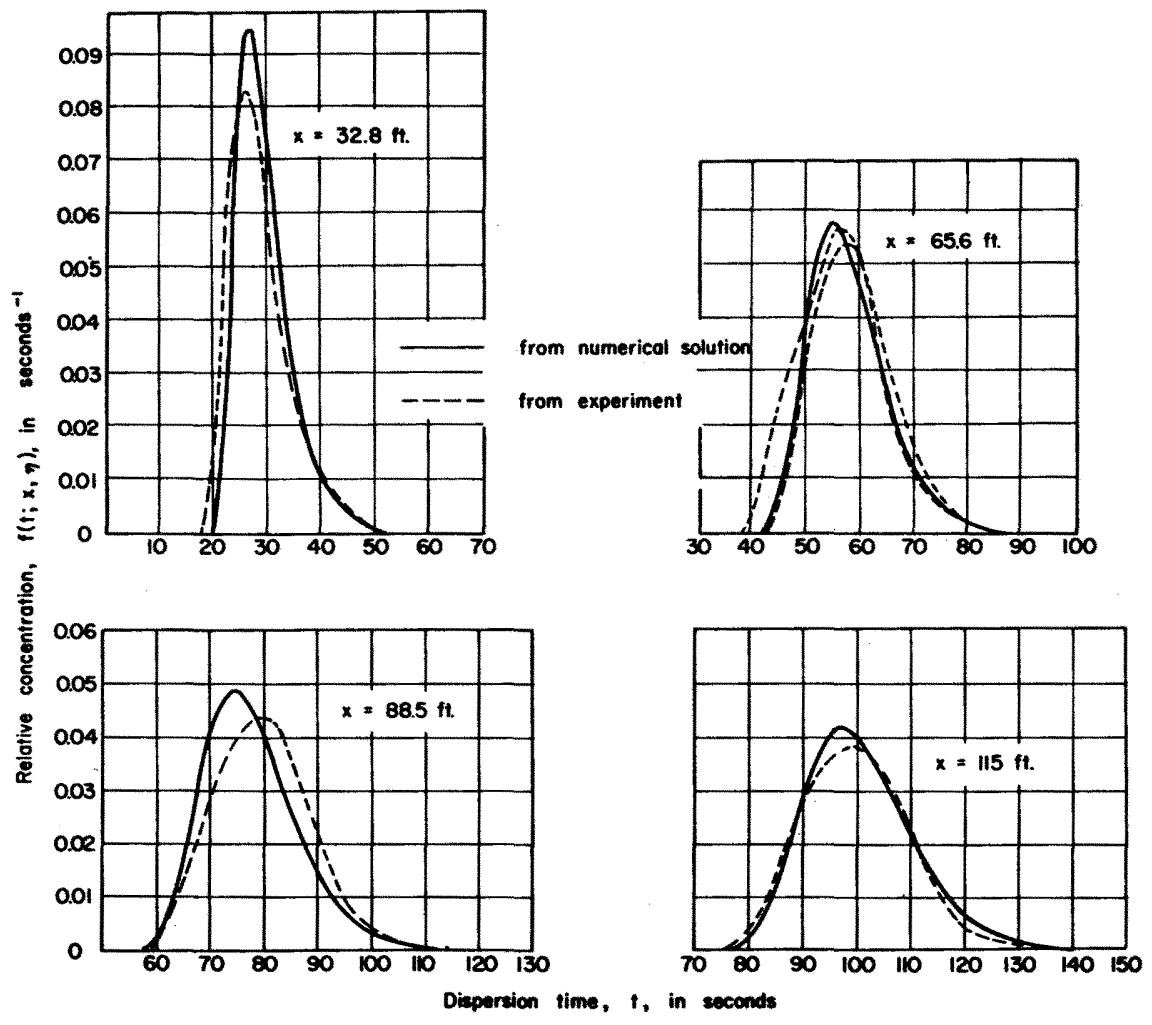

Figure 4-6. Comparison of experimental and approximate theoretical relative concentration versus time curves, longitudinal dispersion of dye, Run D-2 


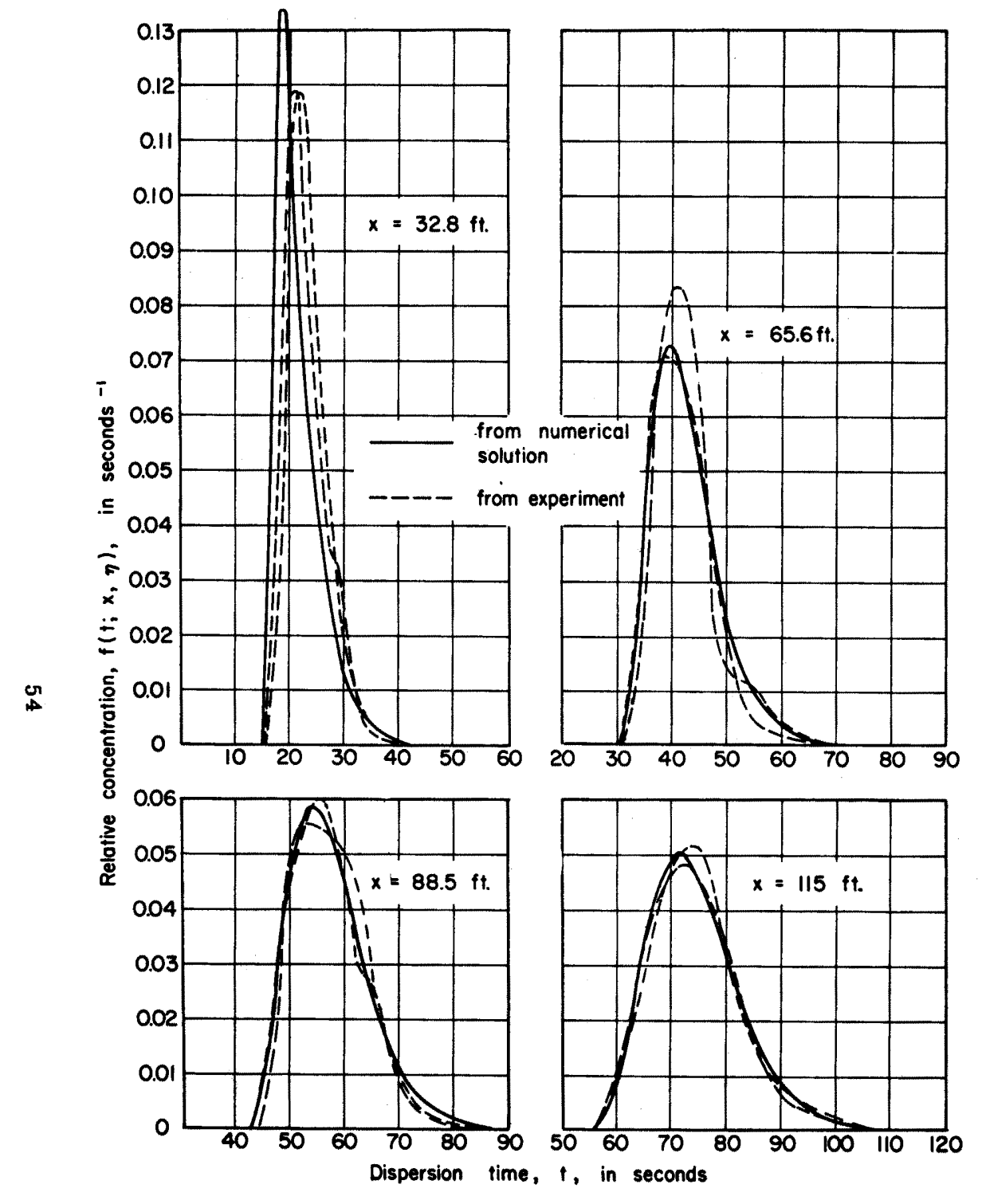

Figure 4-7. Comparison of experimental and approximate theoretical relative concentration versus time curves, longitudinal dispersion of dye, Run D-3
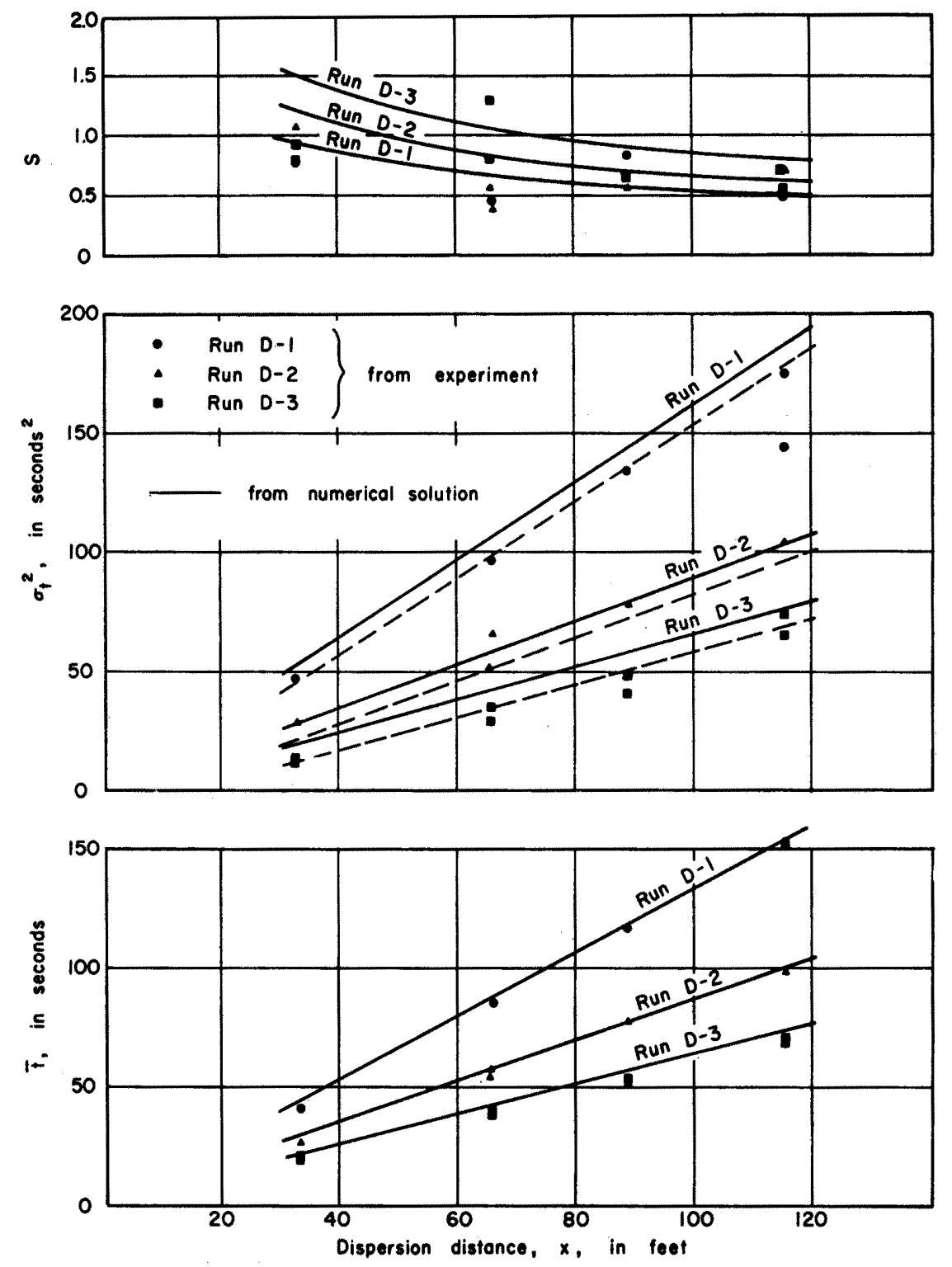

Figure 4-8. Comparison of experimental and approximate theoretical mean travel times, variances and skew coefficients, longitudinal dispersion of dye 


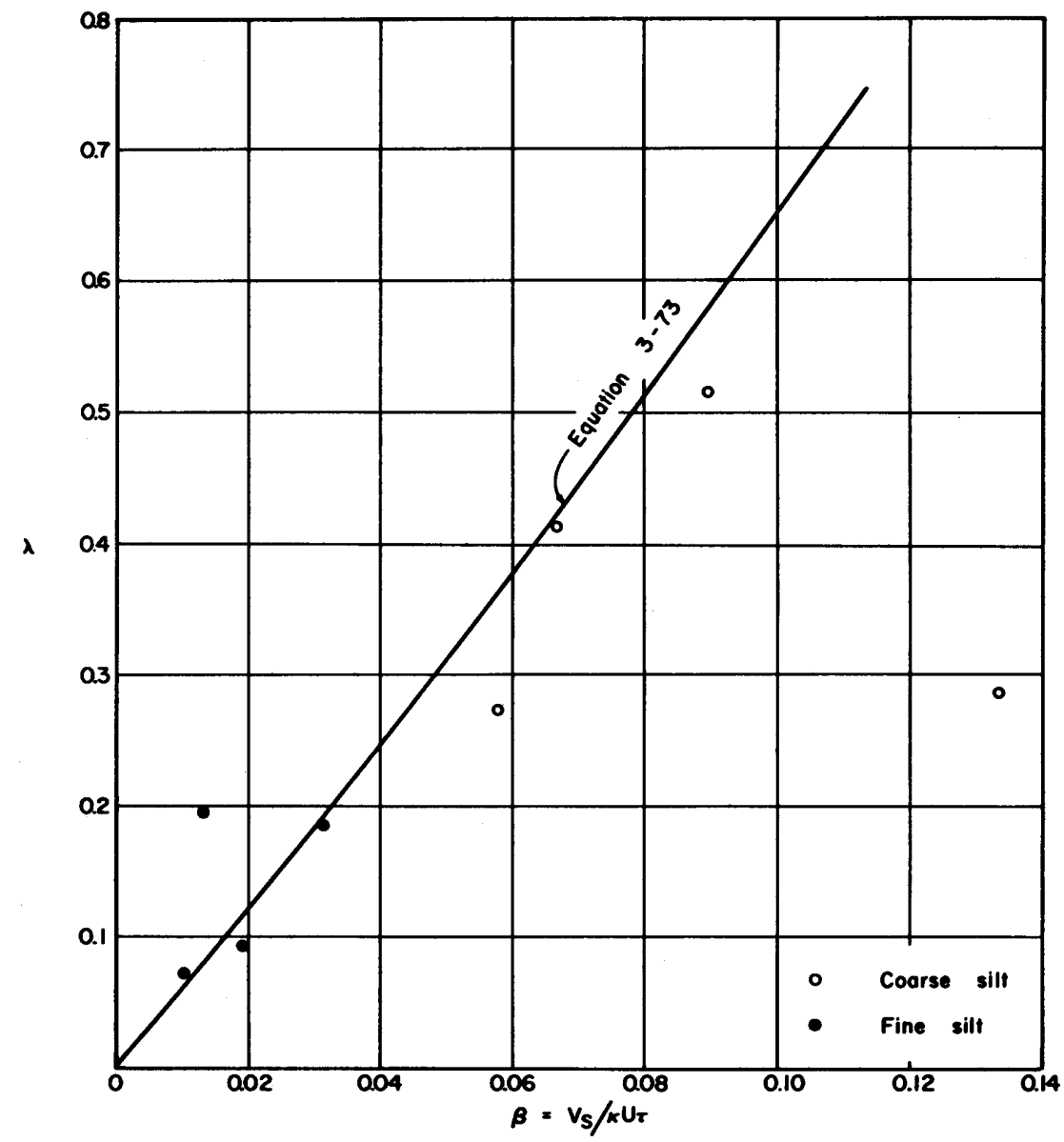

Figure 4-9. Comparison of experimental and theoretical mean rates at which particles settle out of suspension

for re-entrainment to become an important factor. Only in Run FG-1 was more than about 20 percent of the particles deposited. The results of the numerical solutions for conditions corresponding to those in the fine silt experiments also do not deviate from the results for the case with $\beta=0$ by more than a few percent.

With the particles in the $53-62 \mu$ size range, however, significant differences begin to appear. Three sets of experimental relative concentration ver. sus time curves are shown in Figures 4-10, 4-11 and 4-12. The differences can be seen, particularly in Runs CG-2 and CG-3, by comparing these curves with the dye dispersion curves for the corresponding flow conditions in Figures 4-5, 4-6 and 4-7. Especially evident is the growth of the tails of the silt dispersion curves with increasing dispersion distance, which suggests that re-entrainment is playing a significant role.

When deposition and re-entrainment are occurring, the basis for converting the results of the numerical solution to their counterparts in the time domain is much less secure. With the dye dispersion results, the problem was largely circumvented by introducing the Pearson Type III distribution function in an auxiliary capacity. The same procedure does not offer much promise in this case. Even if a general relationship of the parameters $m, b$ and $a$ of the Pearson function to dispersion time were found, it is unlikely that the first three moments of the longitudinal distribution as functions of dispersion time could in themselves provide a sufficiently reliable basis for constructing concentration versus time curves. Therefore, approximations based on Taylor's hypothesis seem to be the only available basis for comparing the numerical solution and experimental results. Bearing in mind the shortcomings of Taylor's hypothesis, the comparisons presented in the following paragraphs, although meaningful in a qualitative sense, may have limited quantitative significance.

In Figure 4-13, recovery ratios are compared. The recovery ratio is defined as the ratio of the amount of dispersant passing a particular sampling location to the amount of dispersant initially carried in suspension. For the experimental results, at the sampling level $n$,

$$
\frac{A(x, \eta)}{A_{0}}=\frac{\int_{0}^{\infty} C(x, \eta, t) d t}{W / Q},
$$




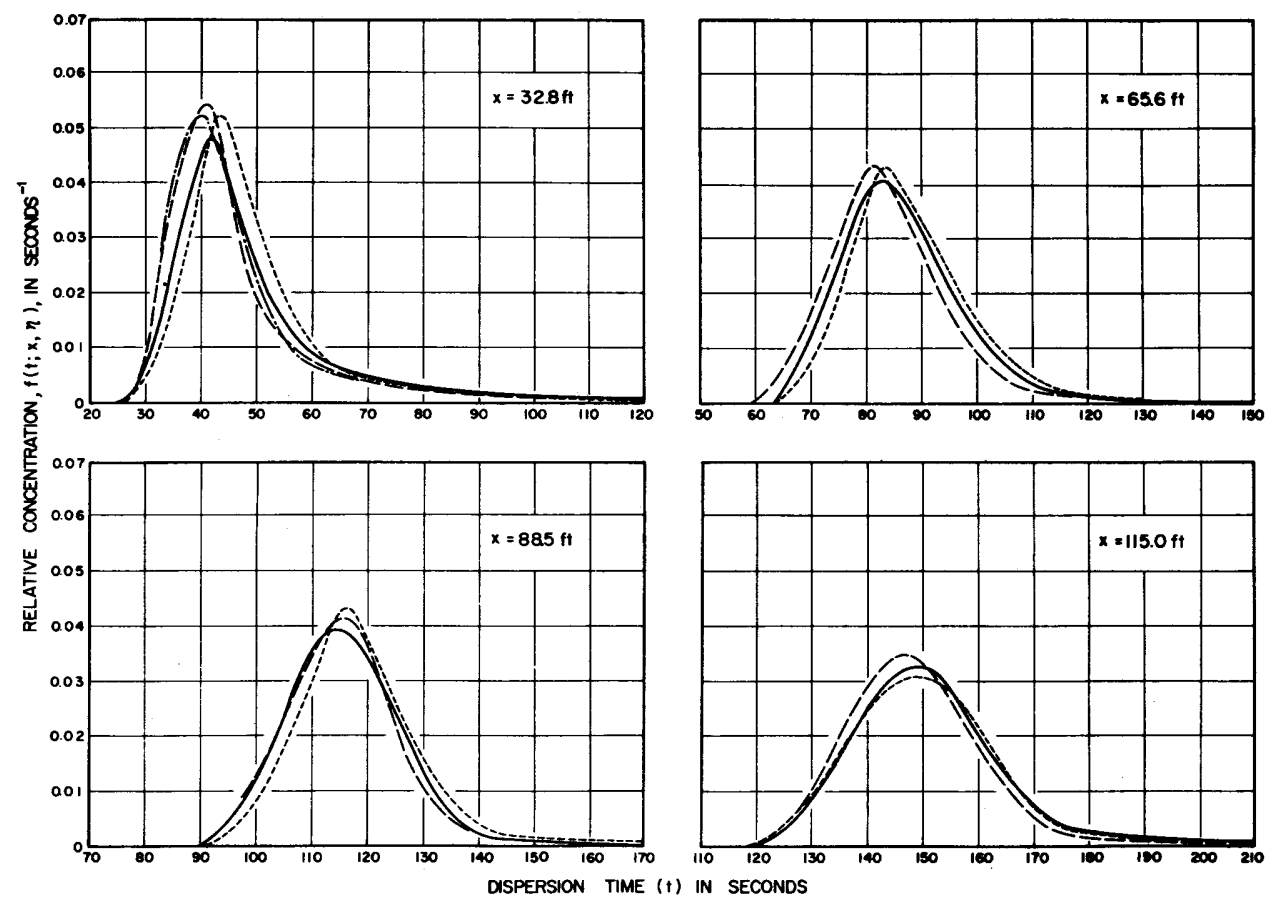

--- OBSERVED AT $\eta=0.25$ OBSERVED AT $\eta=0.50 \quad$-OBSERVED AT $\eta=0.75$ - CONTMNUOUS STSTEM

Figure 4-10. Experimental relative concentration versus time curves, longitudinal dispersion of 53-62 $\mu$ glass beads, Run CG-1
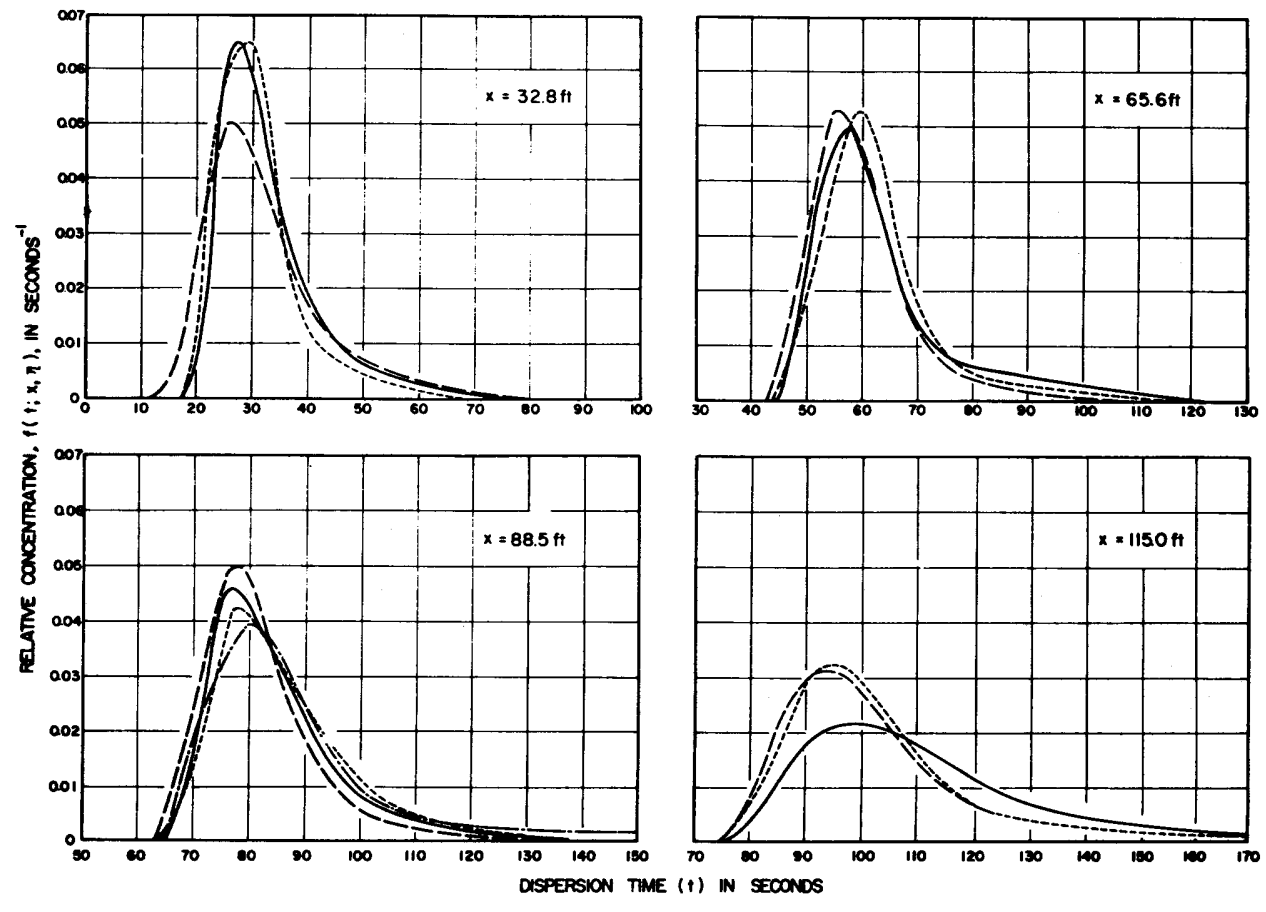

- CBSERVED AT $\eta=0.25$ OBSERVED AT $\eta=050$

OBSERVED AT $\eta=0.75-$ CONTINUOUS SYSTEM

Figure 4-11. Experimental relative concentration versus time curves, longitudinal dispersion of $53-62 \mu$ glass beads, Run CG-2 


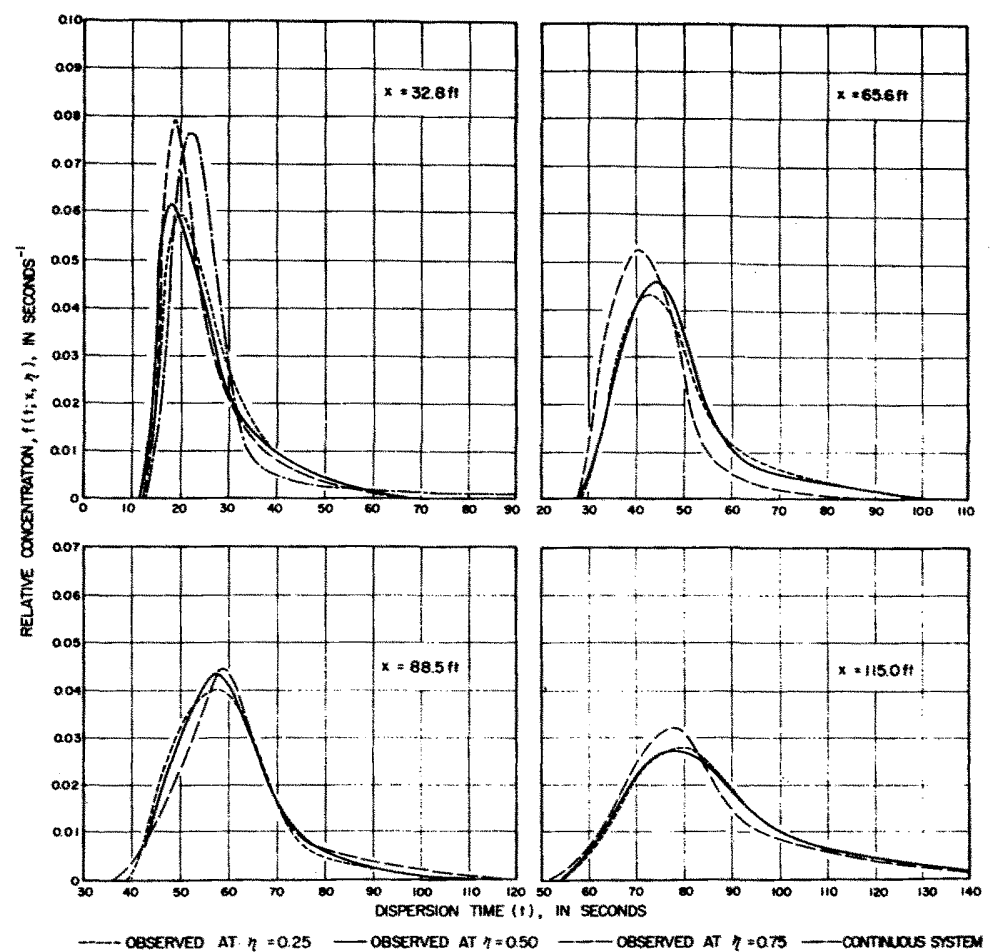

Figure 4-12. Experimental relative concentration versus time curves, longitudinal dispersion of $53-62 \mu$ glass beads, Run CG-3

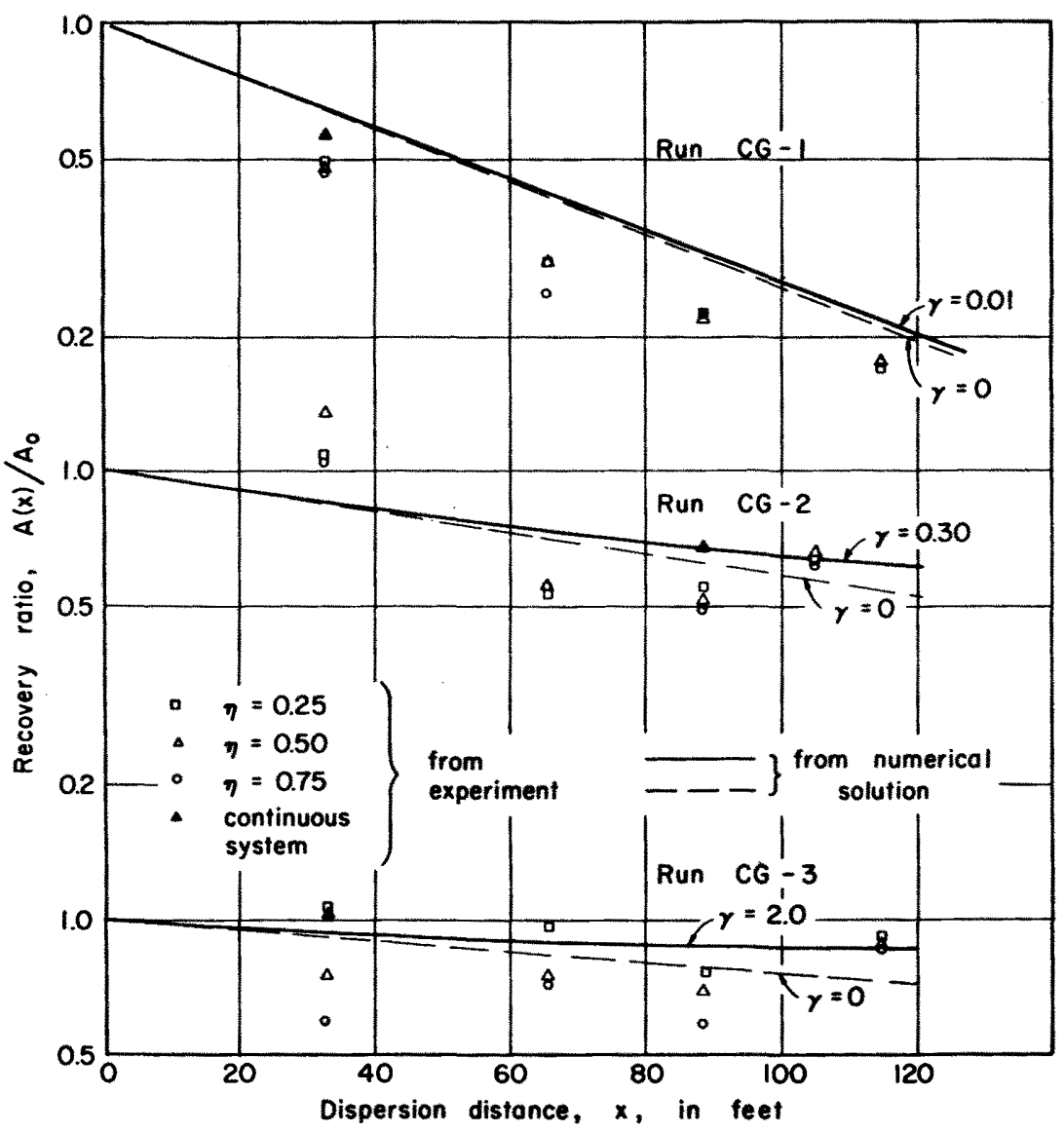

Figure 4-13. Comparison of experimental and approximate theoretical recovery ratios, Runs CG-1, CG-2 and $\mathrm{CG}-3$ 
where $W$ is here the total weight of dispersant, and the numerators represent the area under a concentration versus time curve. For the entire vertical,

$$
\frac{A(x)}{A_{0}}=\frac{1}{A_{0}} \int_{0}^{1} A(x, \eta) d \eta .
$$

For the results of the numerical solution, it was assumed that

$$
m_{0}(\tau) \longrightarrow \frac{A(x)}{A_{0}}
$$

under the approximate transformation

$$
\mathrm{x} \approx \frac{6}{\kappa} \mathrm{y}_{\mathrm{n}} \frac{\overline{\mathrm{U}}}{\mathrm{U}_{\tau}} \tau
$$

which comes from making the substitution $t \approx x / \bar{U}$ in the definition of $\tau$. Considering the scatter of the experimental data and the insensitivity of the recovery ratio to entrainment rate indicated by the results of the numerical solution, not much can be concluded from the comparison other than to observe that both the experimental and theoretical results follow the same general trend.

Comparisons for the mean travel time, $\bar{t}$, are given in Figure 4-14. The results of the numerical solution were transferred to the time domain by means of the approximate relationships, equation 4-6 and

$$
\overline{\mathrm{t}} \approx \frac{36 \overline{\mathrm{Uy}}_{\mathrm{n}} \tau^{2}}{\kappa^{2} \mathrm{U}_{\tau}^{2}\left(\bar{\xi}_{\mathrm{S}}+\mu \tau\right)}
$$

which are again based on Taylor's hypothesis and the conversion factors defined in Equation 2-22. The tendency of the experimental data to lie above the $\gamma=0$ line suggests that re-entrainment is occurring.

By far the most interesting comparison is the one for the variance, $\sigma_{t}^{2}$, shown in Figure 4-15.

Here the approximate relationship

$$
\sigma_{\mathrm{t}}^{2} \approx\left(\frac{\mathrm{y}_{\mathrm{n}}}{\overline{\mathrm{U}}}\right)^{2} \sigma_{\xi}^{2}
$$

together with equation 4-6 was used to transfer the results of the numerical solution to the time domain. For Runs CG-2 and CG-3 values of the entrainment factor $\gamma$ which led to reasonably good agreement with the experimental data were determined by trial and error. The dashed curves above and below represent the maximum and minimum values of variance obtained in the numerical solution for the indicated entrainment factor. These occurred in the bottom and top increments respectively, i.e., for $\eta \approx 0$ and $\eta \approx 1$. As seen in Figures 4-13 and 4-14, curves for these same values of $\gamma$ are not inconsistent with the experimentally-determined recovery ratios and mean travel times. The combined theoretical and experimental results for Runs CG-2 and CG-3 confirm beyond any reasonable doubt the occurrence of the deposition-re-entrainment phenomenon in these runs.

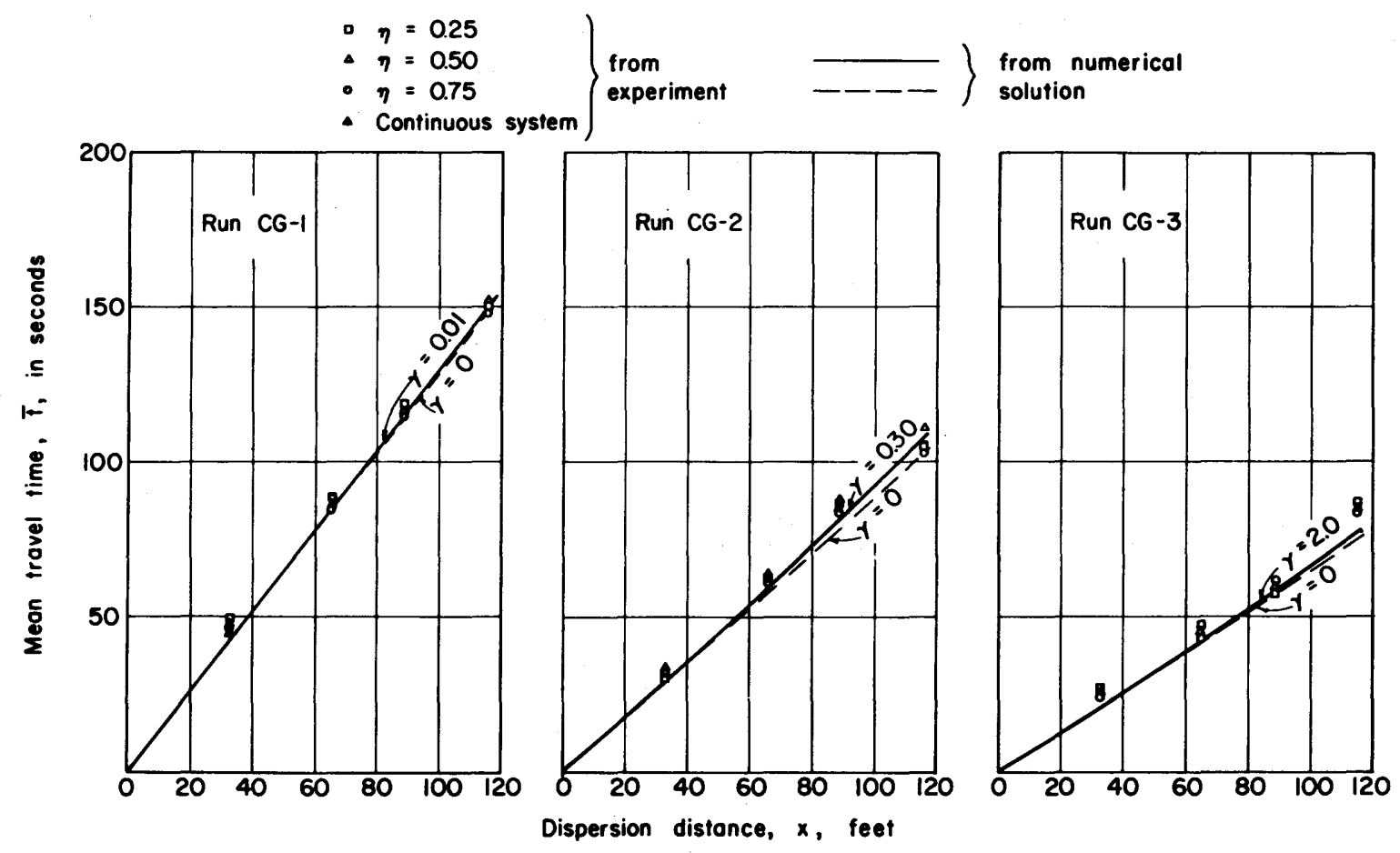

Figure 4-14. Comparison of experimental and approximate theoretical mean travel times, Runs CG-1, CG-2 and CG-3 


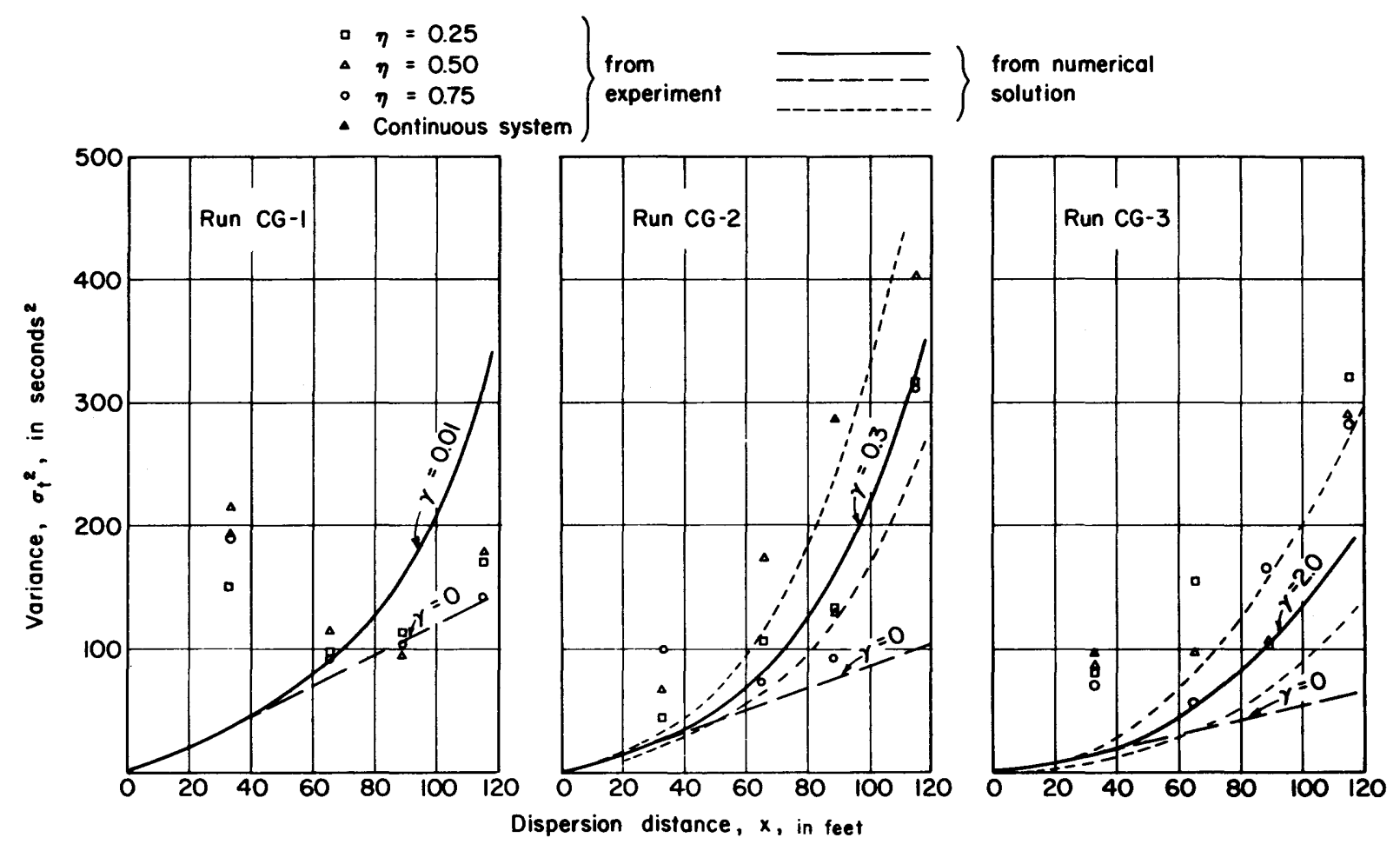

Figure 4-15. Comparison of experimental and approximate theoretical variances, Runs CG-1, CG 2 and CG-3

The rate of re-entrainment in Run CG-1 must have been very small. The tendency of the entrainment rate to increase going from Run CG-1 to CG-3 is certainly consistent with the increase in $U_{\tau}$ and consequent reduction in the thickness of the viscous sublayer which decreases from about eight to about five times the average particle diameter. Because of the error involved in Taylor's hypothesis, however, it is likely that the estimated values of $\gamma$ are too large, perhaps by several fold.
The comparison between the experimental and numerical solution results for the dispersion of particles in the $53-62 \mu$ size range has been limited in scope and qualitative in nature. However, it does suggest that the equations for suspended sediment transport, and deposition and re-entrainment, equations 3-2 and 3-6, with appropriate boundary conditions, are indeed capable of describing the longitudinal dispersion of silt particles in open-channel turbulent shear flow. 


\section{SUMMARY AND CONCLUSIONS}

The Eulerian equation for dispersion in a twodimensional, open-channel, turbulent shear flow, with a known velocity and eddy-diffusivity distribution, has been transformed by the method of Aris (1956) into a set of differential equations for the moments of the longitudinal distribution of dispersing material. Solutions of these equations for the zero'th, first, second and third moments have been obtained, mostly by numerical methods with the aid of a digital computer, for various combinations of boundary and parametric conditions. For a few simple cases, formal analytical solutions were obtained also. From the moments, certain statistical parameters, including the area under the distribution curve, mean displacement, variance, and skew coefficient, were obtained as functions of dispersion time and position in the vertical. These parameters were in turn used to describe the longitudinal dispersion process.

For all cases, the initial condition consisted of an instantaneous plane source, uniformly distributed over the flow cross section. In addition, local values of the eddy diffusivity were in all cases determined by assuming that the mechanisms of mass and momentum transfer in the vertical direction are the same.

For the case of a dissolved dispersant, e.g., a dye having the same fluid properties as the dispersion medium, the effect of velocity distribution on longitudinal dispersion was investigated. For a dispersant consisting of a group of silt-size sediment particles, in a flow with a logarithmic velocity distribution, the effects of the settling velocity of the particles and of various boundary conditions at the bed were investigated. Finally, for situations where sufficient basis for comparison exists, the results of these investigations have been compared with previously established theories and available experimental evidence.

The investigations gave rise to two groups of conclusions, one pertaining to the behavior of dis solved dispersants, and the other to the dispersion of sediment particles.

For dissolved dispersants:

1. As hypothesized by Taylor (1954a) and Elder (1959), longitudinal dispersion is caused by the combined action of turbulent diffusion and differential convection due to the velocity gradient. Turbulent diffusion acts as a catalytic agent by spreading the dispersant vertically so that it is continuously exposed to the effect of the velocity gradient which then becomes the predominant mechanism in promoting longitudinal dispersion.
2. The longitudinal dispersion process can be separated into an initial mixing period and an equilibrium period. During the initial mixing period, the distribution of local mean displacement and variance with respect to depth changes with time. After a dimensionless dispersion time of

$$
\tau=\frac{\mathrm{Dt}}{\mathrm{y}_{\mathrm{n}}^{2}} \approx 0.5
$$

these distributions become stable, supporting the intuitive hypothesis of Taylor (1954a) and Elder (1959) that a balance between the longitudinal convective and vertical diffusive transfer rates is attained.

3. In the equilibrium period, the longitudinal dispersion process becomes essentially one dimensional, in some respects resembling classical Fickian diffusion. The rate of growth of the variance is constant, indicating the existence of a constant overall longitudinal dispersion coefficient. The rate of approach from negative skewness to symmetry is quite slow, however, indicating that the longitudinal concentration distribution converges to the form of the normal probability density function only at asympotically large dispersion times.

4. For a logarithmic velocity distribution the value of the longitudinal dispersion coefficient in the equilibrium period is

$$
\mathrm{K}_{\mathrm{x}}=\left[\frac{0.40}{\kappa^{3}}+\frac{\kappa}{6}\right] \mathrm{y}_{\mathrm{n}} \mathrm{U}_{\tau}
$$

which agrees with the value predicted by Elder (1959) for large dispersion times. For a parabolic velocity distribution function that was also investigated, the value of the numerical coefficient in the equation for $\mathrm{K}_{\mathrm{X}}$ was different. In other respects, however, only relatively minor differences were found.

5. The longitudinal dispersion process can be interpreted in terms of Taylor's (1921) theory of diffusion by continuous movements. Considering a flow with a logarithmic velocity distribution and neglecting the direct contribution of turbulent diffusion to longitudinal dispersion, the Lagrangian intensity of turbulence is

$$
\overline{u^{\prime 2}}=\left(\frac{U_{T}}{\kappa}\right)^{2},
$$

and the Lagrangian integral time scale of turbulence in dimensionless time units is $L_{T} \approx 0.07$. 
For silt-size sediment particles:

6. Provided that there is no re-entrainment of deposited particles, the longitudinal dispersion of sus pended particles in most respects closely resembles the longitudinal dispersion of a dissolved substance. There are, however, significant differences due to the tendency of the particles to settle and the consequent development of a vertical concentration gradient. The degree of difference increases systematically with the settling velocity parameter, $\beta=\mathrm{V}_{\mathbf{S}} / \kappa \mathrm{U}_{\tau}$.

7. When the bed behaves as a reflecting barrier, the rate of dispersion is somewhat greater, and the mean velocity of propagation and the skewness are somewhat less for the particles than for a dissolved dispersant.

8. When the bed behaves as an absorbing barrier, the amount of sediment retained in suspension decreases exponentially with dispersion time.
For the particles retained in suspension, the mean velocity of propagation is somewhat greater, and the rate of dispersion and the skewness are less than for a dissolved dispersant. The longitudinal distribution of the particles which are deposited on the bed converges to an exponential distribution with increasing dispersion time.

9. When re-entrainment of deposited particles occurs, radical changes may appear in the longitudinal dispersion process, whether for the suspended component, the deposited component, or the composite of both. Paramount among these is a change in the rate of dispersion which under some conditions is greatly increased. In addition, the duration of the initial mixing period is apt to be greatly extended because the equilibrium period cannot be attained before the deposition and re-entrainment rates are balanced. Given the velocity distribution, the controlling parameters are the entrainment coefficient, $\gamma$; the fall velocity parameter, $\beta$; and the mean flow velocity, $\mu$. 


\section{SUGGESTIONS FOR FURTHER RESEARCH}

1. Numerical solutions of the moment equations should be performed for simulated three-dimensional flows in which the velocity and eddy diffusivity vary with lateral as well as with vertical position. The possibility of using this method for investigating dispersion in flows with secondary circulation patterns and in channels of various cross-sectional shapes should be explored.

2. The effect of the initial distribution of dispersant should be investigated by obtaining solutions corresponding to different initial source configurations. Solutions for point sources would be of particular interest. With these, solutions corresponding to other source configurations can be constructed by superposition.

3. Practical experimental techniques for obtaining instantaneous longitudinal concentration dis tributions should be developed. The simplest way may be to develop a device for obtaining a group of instantaneous grab samples simultaneously. Fifteen or more samples, distributed along the length of the channel, would be required to adequately define a concentration distribution curve. Ideally, the device should be capable of collecting samples at any point in the cross section, and should be suitable for particulate as well as for dissolved dispersants.

4. A great deal of research, both experimental and analytical, remains to be done on the entrainment of sediment. In addition to the rate of entrainment, the duration of the rest periods between the deposition and re-entrainment of a given particle needs to be determined. This involves considerations such as the geometrical configuration of sediment deposits and mixing processes occurring within the bed, which go considerably beyond the mechanics of lift and drag forces. In other words, to evaluate the probability that a particle will be entrained within a given period of time, not only the conditional probability that it will be entrained given that it is exposed to an impulse of a specified intensity, but also the probability of exposure must be known.
5. The phenomenon of local mass transfer in open-channel turbulent shear flows and its relationship to momentum transfer should be investigated further with a view toward defining the limitations of Reynolds' analogy. For dispersants consisting of particles above the Stokes range, the need for this kind of information is especially great.

6. Further investigations of the effect of openchannel, shear-flow turbulence on the mean fall velocity of sediment particles should also be undertaken. An approach which appears promising would be to compare deposition rates which are determined experimentally, with those predicted by the zero'th moment equation.

7. A computer program should be written for the numerical solution of the Eulerian dispersion equation for suspended sediment (equation 3-2) together with the conservation of mass equation for the bed (equation 3-6). Recognizing that a single run might require hours of computer time, even on a third generation model, considerable selectivity should be exercised in choosing boundary conditions and other input data. Results for a few well-selected cases could serve as benchmarks for interpreting results obtained from the moment equations, which in contrast require a nominal computation time.

\section{The feasibility of generalizing the} relationships for the parameters describing the longitudinal distribution of dispersing sediment when re-entrainment is occurring should be investigated. A possible approach would be to incorporate the fall velocity term $\beta$, and the entrainment rate coefficient $\gamma$, into the time scale by dimensional analysis.

9. By introducing the fourth central moment, in addition to the first three, it may be possible, through the use of Pearson's criteria, to find longitudinal distribution functions which correspond more closely to the numerical solutions of the Aris equations than does the Pearson Type III function. 


\section{BIBLIOGRAPHY}

Al-Saffar, A. M., 1964, Eddy diffusion and mass transfer in open-channel flow: Ph. D. Dissertation, University of California, Berkeley, $138 \mathrm{p}$.

Ananthakrishnan, V., Gill, W. N. and Barduhn, A. J. 1965, Laminar dispersion in capillaries: Part $I$, mathematical analysis: Am. Inst. Chem. Engr. Journal, Vol. 11, No. 6, p. 1063-1072.

Aris, R., 1956, On the dispersion of a solute in a fluid flowing through a tube: Proc. Royal Soc. of London, Vol. 235A, p. 67-77.

Bailey, H. R. and Gogarty, W. B., 1962, Numerical and experimental results on the dispersion of a solute in a fluid in laminar flow through a tube: Proc. Royal Soc. of London, Vol. 269A, p. 352367.

Batchelor, G. K., and Townsend, A. A., 1956, Turbulent diffusion: p. 352-399 in Surveys in Mechanics, edited by G. K. Batchelor and R. M. Davies, Cambridge University Press, $475 \mathrm{p}$.

Camp, T. R., 1944, Discussion of "Effect of turbulence on sedimentation": Trans. Am. Soc. Civil Engineers, Vol. 109, p. 660-667.

Camp, T. R., 1946, Sedimentation and the design of settling tanks: Trans. Am. Soc. Civil Engineers, Vol. 111, p. 895-958.

Carslaw, H. S. and Jaeger, J. C., 1959, Conduction of heat in solids: Oxford University Press London, 2nd Edition, $510 \mathrm{p}$.

Churchill, R. V., 1941, Fourier series and boundary value problems: McGraw Hill Book Co., New York, 206 p.

Crank, J., 1956, The mathematics of diffusion: Oxford University Press, London, 347 p.

Dobbins, W. E., 1944, Effect of turbulence on sedimentation: Trans. Am. Soc. Civil Engineers, Vol. 109, p. 629-678.

Einstein, H. A., 1937, Der Geschiebetrieb als Wahrscheinlichkeitsproblem: Zurich, Verlag Rascher, 110 p.

Elder, J. W., 1959, The dispersion of marked fluid in turbulent shear flow: Journal of Fluid Mechanics, Vol. 5, No. 4, p. 544-560.

Evans, R. D., 1955, The atmoic nucleus: McGrawHill Book Co., 972 p.
Fischer, H. B., 1964, Longitudinal dispersion by velocity gradients in open channel flow: Technical Memo 64-4, W. M. Keck Laboratory, California Institute of Technology, $12 \mathrm{p}$.

Fischer, H. B., 1966, Longitudinal dispersion in laboratory and natural streams: Report No. $\mathrm{KH}-\mathrm{R}-12$, California Inst. of Technology, Pasadena, $250 \mathrm{p}$.

Galvin, C. J., Jr., 1965, Discussion of "Sand transport studies with radioactive tracers": Proc. Am. Soc. Civil Engineers, Vol. 91, No. HY1, p. 173-178.

Glover, R. E., 1964, Dispersion of dissolved or suspended materials in flowing streams: U.S. Geol. Survey Prof. Paper 433-B, 32 p.

Godfrey, R. G., and Frederick, B. J., 1963, Dispersion in natural streams: U. S. Geological Survey Open File Report, Washington, D. C. , $75 \mathrm{p}$.

Hays, J. R., Krenkel, P. A. and Schnelle, K. B., Jr., 1966, Mass transport mechanisms in openchannel flow: Technical Report No. 8, Sanitary and Water Resources Engrg., Dept. of Civil Engrg., Vanderbilt University, Nashville, 138 p.

Hinze, J. O., 1959, Turbulence: McGraw-Hill Book Co., New York, 586 p.

Hubbell, D. W. and Sayre, W. W., 1964, Sand transport studies with radioactive tracers: Am. Soc. Civil Engineers Proc., Vol. 90, No. HY4, p. 39-68.

Hunt, J. N., 1954, The turbulent transport of suspended sediment in open channels: Proc. Royal Soc. of London, Vol. 224A, p. 322-335.

Kalinske, A. A., and Pien, C. L., 1944, Eddy diffusion: Industrial and Engineering Chemistry, Vol, 36, p. 220-223.

Lane, E. W. and Kalinske, A. A., 1939, The relation of suspended to bed material in rivers: Transactions, American Geophysical Union, Vol. 20, p. 637-641.

Laufer, J., 1951, Investigation of turbulent flow in a two-dimensional channel, N. A.C.A. Report No. 1053.

McLaughlin, R. T., 1959, The settling properties of suspensions: Proc. Am. Soc. Civil Engineers, Vol. 85 , No. HY12, p. 9-41. 
Mickelsen, W. R., 1960, Measurements of the effect of the molecular diffusivity--turbulent diffusion: Journal of Fluid Mechanics, Vol. 7, p. 397-400.

Orlob, G. T., 1958, Eddy diffusion in open channel flow: Contribution No. 19, Sanitary Engineering Research Laboratory, University of California, $144 \mathrm{p}$.

Orlob, G. T., 1961, Eddy diffusion in homogeneous turbulence: Trans. Am. Soc. Civil Engineers, Vol. 126, Part I, p. 397-438.

Reichardt, H., 1938, Messungen turbulenter Schwankungen: Naturwissenschaften, 404.

Richtmyer, R. D., 1957, Difference methods for initial-value problems: Interscience Publishers Inc., New York, 238 p.

Rohsenow, W. M. and Choi, H. Y., 1961, Heat, mass and momentum transfer: Prentice-Hall, Englewood Cliffs, N. J., 529 p.

Rouse, Hunter, 1937, Closure to "Modern conceptions of the mechanics of fluid turbulence": Trans. Am. Soc. Civil Engineers, Vol. 102, p. 534-536.

Sayre, W. W., and Chang, F. M., 1966, A laboratory investigation of open-channel dispersion processes for dissolved, suspended and floating dispersants: U.S. Geol. Survey, Water Resources Div., Open File Report, Fort Collins, Colorado, $210 \mathrm{p}$.

Singamsetti, S. R., 1966, Diffusion of sediment in a submerged jet: Proc. Am. Soc. Civil Engineers, Vol. 92, No. HY2, p. 153-168.

Task Committee on Preparation of Sedimentation Manual, 1963, Sediment transportation mechanics: Suspension of sediment: Proc. Am. Soc. Civil Engineers, Vol. 89, No. HY5, p. 45-87.
Taylor, G. I., 1921, Diffusion by continuous movements: Proc. London Math. Soc., A20, p. 196211.

Taylor, G. I., 1953, Dispersion of soluble matter in solvent flowing slowly through a tube: Proc. Royal Soc. London, Vol. 219A, p. 186-203.

Taylor, G. I., 1954a, The dispersion of matter in turbulent flow through a pipe: Proc. Royal Soc. London, Vol. 223A, p. 446-468.

Taylor, G. I., 1954b, Conditions under which dispersion of a solute in a stream of solvent can be used to measure molecular diffusion: Proc. Royal Soc. London, Vol. 225A, p. 473-477.

Tchen, C. M., 1947, Mean value and correlation problems connected with the motion of small particles suspended in a turbulent fluid: Publ. 51 of Lab for Aero- and Hydrodynamics, Technical University, Delft.

Vanoni, V. A., 1946, Transportation of suspended sediment by water: Trans. Am. Soc, Civil Engineers, Vol. 111, p. 67-133.

Yotsukura, Nobuhiro, 1963, Turbulent dispersion of miscible materials in open channels: p. 311-326 in "Transport of radionuclides.in fresh water systems, " U.S. Atomic Energy Comm. Report No. T1D-7664, 406 p.

Yotsukura, Nobuhiro, Smoot, G. F., and Cahal, D. I., 1964, Dispersion in open channel flow: A contribution to the Vicksburg ASCE Hydraulics Div. Conf., U.S. Geol. Survey, Water Resources Div., 19 p.

Yotsukura, Nobuhiro, and Fiering, M. B., 1964, Numerical solution to a dispersion equation: Proc. Am. Soc., Civil Engineers, Vol. 90, No. HY 5, p. 83-104.

Yotsukura, Nobuhiro and Fiering, M. B., 1966, Closure to "Numerical solution to a dispersion equation": Proc. Am. Soc. Civil Engineers, Vol. 92, No. HY3, p. 67-72. 
COMPUTER PROGRAMS AND SUPPLEMENTARY INFORMATION

SELECTED VARIABLE NAMES USED IN PROGRAM I

\begin{tabular}{|c|c|}
\hline Variable Name & Term Represented \\
\hline DT & $\Delta \boldsymbol{T}$ \\
\hline DY & $\Delta \eta$ \\
\hline KI & $1 / \Delta \eta$ \\
\hline $\mathbf{K 2}$ & No. of $\Delta t$ steps in program \\
\hline K3 & No. of $\Delta \tau$ steps between print outs \\
\hline $\mathrm{E}(\mathrm{I})$ & equation $3-25$ \\
\hline $\mathrm{U}(\mathrm{I})$ & equation $3-23$ \\
\hline $\mathrm{C}(\mathrm{I}, \mathrm{J})$ & $\kappa^{2} \mathrm{C}_{1}(\mathrm{I}, \mathrm{J})$ \\
\hline DL & $\frac{D \kappa^{3}}{\mathrm{y}_{\mathrm{n}} \mathrm{U}_{\tau}} \mathrm{K}_{\mathrm{C}}(\tau)$ \\
\hline
\end{tabular}

PROGRAM I

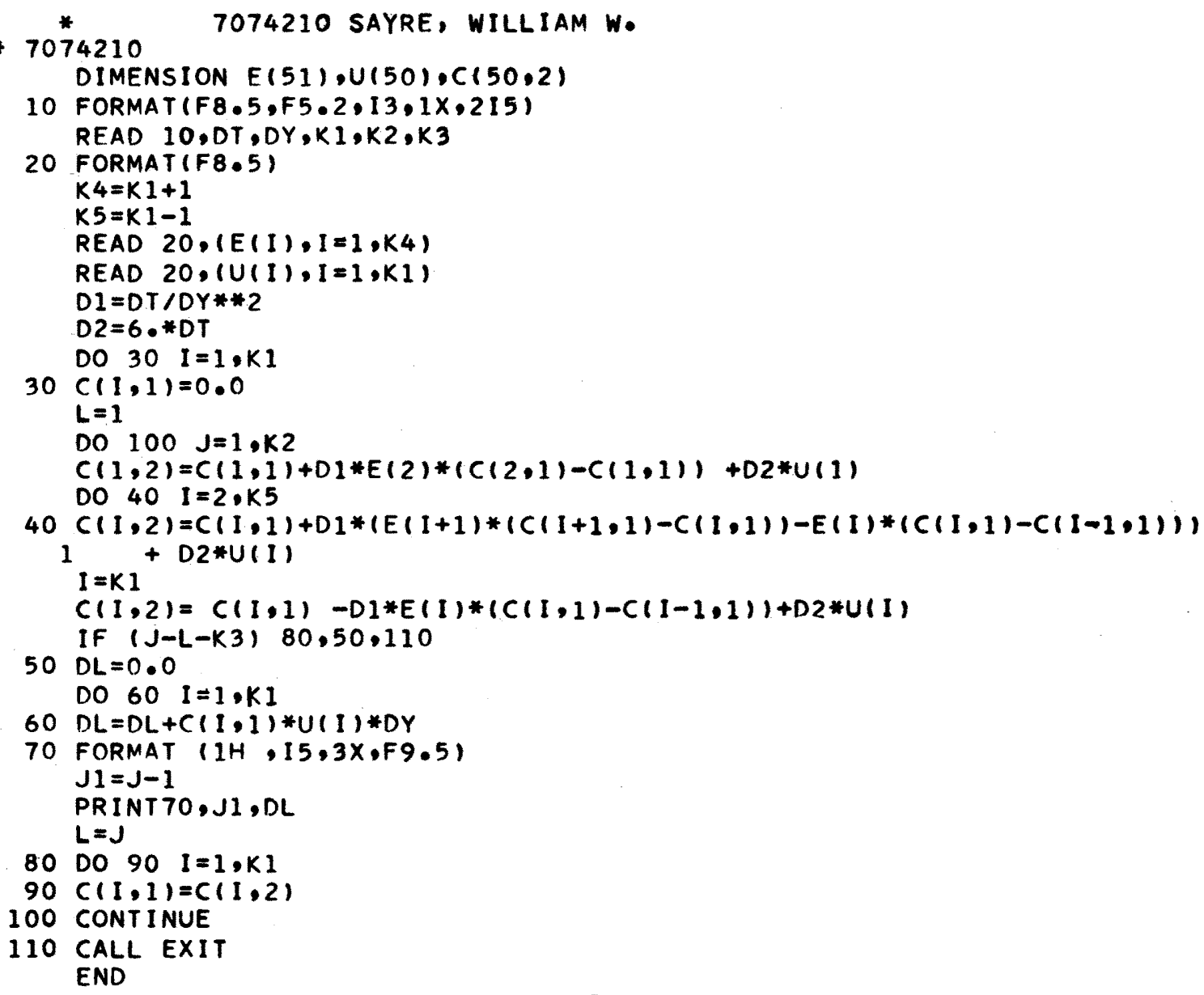


- FORTRAN

PROGRAM SAYRE

DIMENSION E(51),U(50), EA(50), CO(50,2), C1 $(50,2), C 2(50,2), E C 1(50)$,

$1 \operatorname{VAR}(50), 02(50), 03(50), 04(50), 05(50), C 3(50,2), S(50), 06(50)$,

2 HO(2),H1(2),H2(2),W3(2)

$X T I M I=T I M E F(X)$

10 FORMAT (F8.5,F5.2,13,1 $1 \times, 215)$

READ $(5,10)$ DT,OY,K1,K2,K3

20 FORMAT (3F6.3,F8.5,F7.3)

25 FORMAT $(1 \mathrm{HO}, 5 \mathrm{H} T=, \mathrm{F} 6.3,5 \mathrm{H}, B=, F 6.3,5 \mathrm{H}, A=, F 6.3,5 \mathrm{H}, G=, F 8.5 \mathrm{~g}$.

I $6 \mathrm{H}, U A=, F 7.3 / 11$ $K 4=K 1+1$

$K 5=K 1-1$

30 FORMAT (F8.5, $3 x, F 8,5,3 x, F 8.5)$

READ $(5,30)$ (E(I),U(I),EA(I), I =1,RI)

35 FORMAT (F8.5)

READ $(5,35)$ E(K4)

DI $=D T / D Y$

36 READ $(5,20) T, B, A, G, U A$

IF $(T-9.999) 37,165,170$

37 MRITE $(6,25)$ T,B,A,G,UA

$D 7=01 * 6 . * B *(1 .-A)$

$D 8=D T * A * 6 . * B$

$D 9=6 . * D T * U A / T$

$G 1=01 * G$

$G 2=D T * G$

$00 \quad 40 \quad I=1, K 1$

$D 2(I)=E(I+1) / O Y+6 . * B$

$D 3(I)=E(I) / O Y$

$D 4(I)=6 . * D T * U(I) / T * * 2$

$D S(I)=2$. $D T * E A(I)$

$D 6(I)=6 . * U(I) / T * * 2$

$\operatorname{CO}(I, 1)=1.0$

$C(1,1)=0.0$

$C 2(1,1)=0.0$

$40 C 3(1,1)=0.0$

$H O(1)=0.0$

$H 1(1)=0.0$

$W_{2}(1)=0.0$

$H 3(1)=0.0$

$L=1$

DO $160 J=1, K 2$

I = I

$\operatorname{co}(1,2)=\operatorname{co}(1,1)+01 *(02(1) *(\operatorname{Co}(1+1,1)-\operatorname{co}(1,1)))+07 * \operatorname{co}(1,1)$

$1+61 *$ WO(1)

DO $50 \quad I=2, K 5$

$50 \operatorname{CO}(1,2)=\operatorname{CO}(1,1)+D 1 *(02(1) *(\operatorname{CO}(1+1,1)-\operatorname{CO}(1,1))-D 3(1) *(\operatorname{CO}(1,1)-$

$(\operatorname{co}(1-1,1) 1)$

$I=K I$

$C O(1,2)=\operatorname{CO}(1,1)-D 1 *(D 3(1) *(\operatorname{CO}(1,1)-\operatorname{CO}(1-1,1))+6 . B * C O(1,1))$

$W O(2)=W O(1)+D 8 * C O(1,1)-G 2 * W O(1)$

$I=1$

$C_{1}(1,2)=C 1(1,1)+D 1=(D 2(1) *(C 1(1+1,1)-C 1(1,1))+D 4(1)=C O(1,1)$

$1+07 * C 1(1,1)+61 * H 1(1)$

DO $80 \quad I=2, K 5$

$80 C 1(1,2)=C 1(1,1)+D 1=(02(1)=(C 1(1+1,1)-C 1(1,1))-D 3(1)=(C 1(1,1)-$

$1 C 1(1-1,1) 1)+04(1)=C O(1,1)$

$I=K I$

$\left.C_{1}(1,2)=C 1(1,1)-D 1 *(D 3(1) *(C 1(1,1)-C(1)-1,2))+6 . * B * C 1(1,1)\right)$

$1+04(1)=C O(1,1)$ 


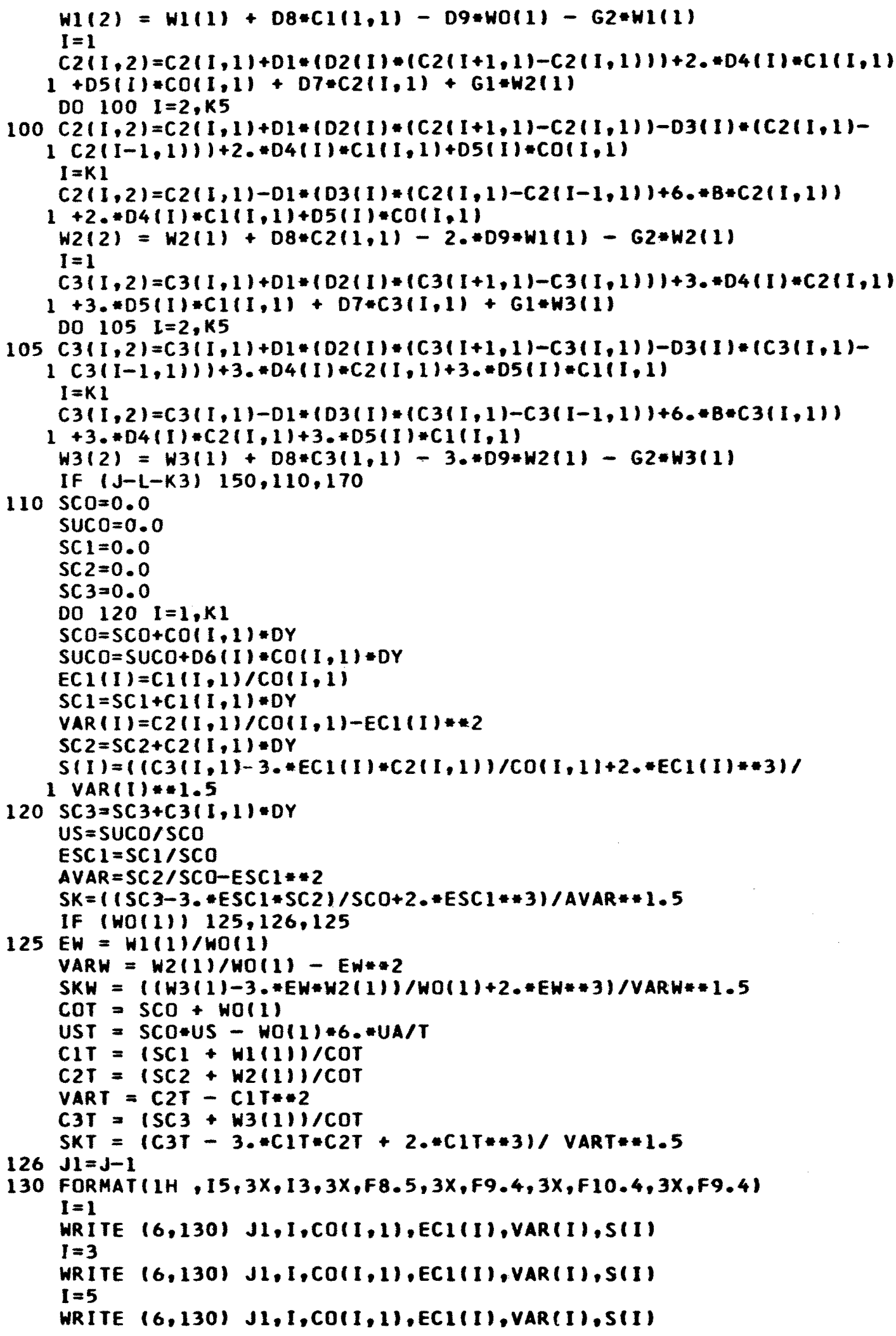




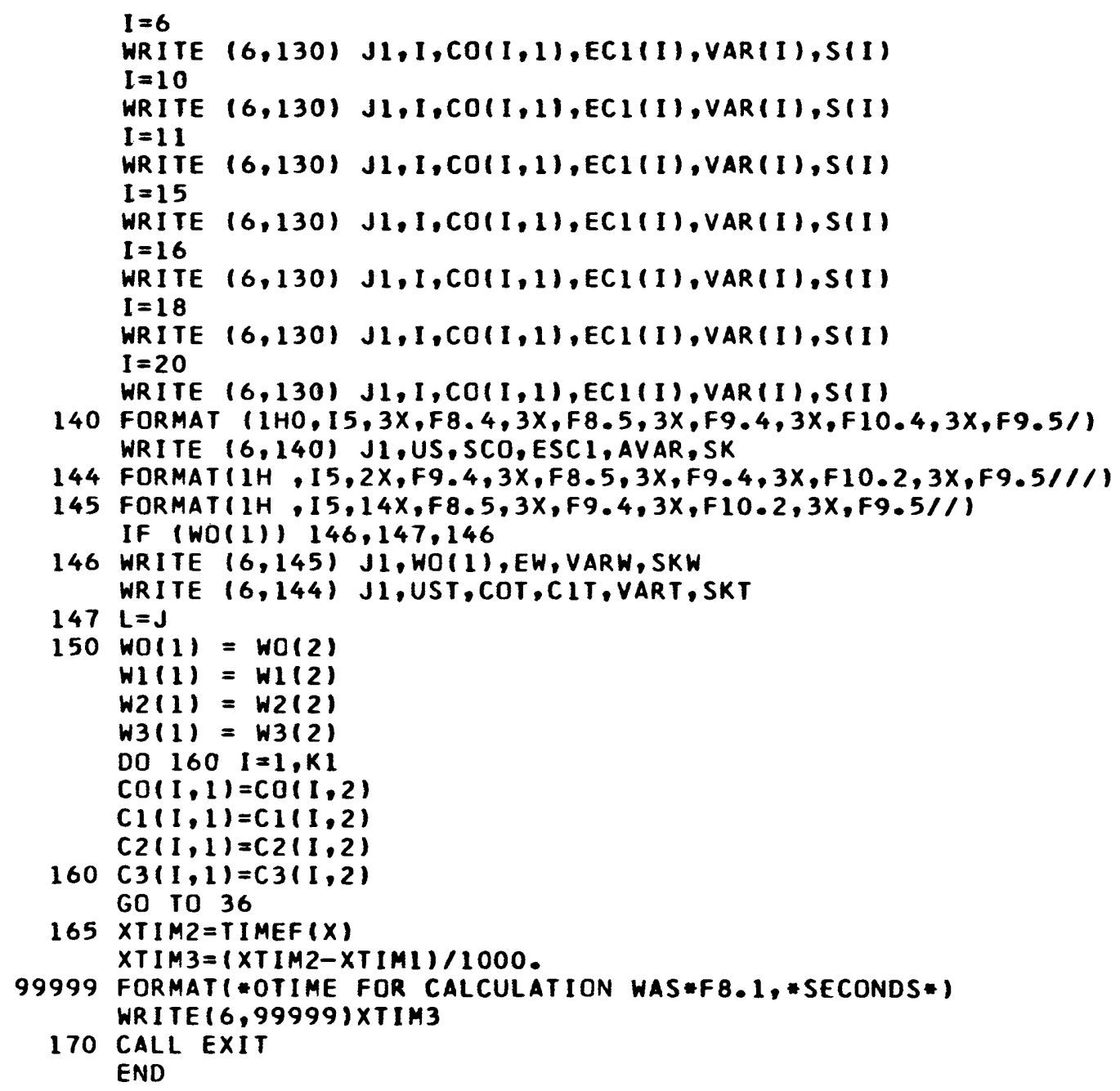




\section{SELECTED VARIABLE NAMES USED IN PROGRAM II}

Variable Name

DT

DY

K1

$\mathrm{K} 2$

K3

$E(I)$

$\mathrm{U}(\mathrm{I})$

EA(I)

$\mathrm{T}$

B

A

G

UA

$\mathrm{CO}(\mathrm{I}, \mathrm{J})$

C1(I, J)

C2(I, J)

C3(I, J)

WO(J)

W 1(J)

W2(J)

W3(J)

SCO

SUCO

EC1(I)

$\mathrm{SC} 1$

$\operatorname{VAR}(\mathrm{I})$

$\mathrm{SC2}$

\section{Term Represented}

$\Delta \tau$

$\Delta \eta$

$1 / \Delta \eta$

No. of $\Delta t$ steps in program

No. of $\Delta \tau$ steps between print outs

equation 3-25

equation 3-23

equation 3-24

$\kappa$

$\beta$

$\alpha$

$\gamma$

$\overline{\mathrm{U}} / \mathrm{U}_{\tau}$

$\mathrm{C}_{0}(\eta, \tau)$

$\mathrm{C}_{1}(\eta, \tau)$

$\mathrm{C}_{2}(\eta, \tau)$

$\mathrm{C}_{3}(\eta, \tau)$

$\mathrm{W}_{0}(\tau)$

$\mathrm{W}_{1}(\tau)$

$\mathrm{W}_{2}(\tau)$

$\mathrm{w}_{3}(\tau)$

$\mathrm{m}_{0}(\tau)$

$\frac{6}{\kappa^{2}} \sum^{\mathrm{K} 1} \mathrm{C}_{0}(\mathrm{I}, \mathrm{J}) \mathrm{U}(\mathrm{I}) \Delta \eta$

$\bar{\xi}_{S}(\eta, \tau)$

$\sum_{\mathrm{I}=1}^{\mathrm{K} 1} \mathrm{C}_{1}(\mathrm{I}, \mathrm{J}) \Delta \eta$

$\sigma_{S}^{2}(\eta, \tau)$

$\sum_{\mathrm{I}=1}^{\mathrm{K} 1} \mathrm{C}_{2}(\mathrm{I}, \mathrm{J}) \Delta \eta$ 
Variable Name

S(I)

SC3

US

ESC1

AVAR

SK

EW

VARW

SKW

US T

COT

C1 T

VART

SKT
Term Represented

$$
\sum_{I=1}^{K 1} \mathrm{C}_{3}(\mathrm{I}, \mathrm{J}) \Delta \eta
$$$$
\bar{\mu}_{\mathrm{s}}(\tau)
$$$$
\bar{\xi}_{S}(\tau)
$$$$
\sigma_{S}^{2}(\tau)
$$$$
\mathrm{S}_{\mathrm{S}}(\tau)
$$$$
\bar{\xi}_{\mathrm{W}}(\tau)
$$$$
\sigma_{\mathrm{W}}^{2}(\tau)
$$$$
\mathrm{S}_{\mathrm{W}^{(\tau)}}
$$$$
\bar{\mu}_{\mathrm{T}}(\tau)
$$$$
\mathrm{m}_{0}(\tau)+\mathrm{W}_{0}(\tau)
$$$$
\bar{\xi}_{\mathrm{T}}(\tau)
$$$$
\sigma_{\mathrm{T}}^{2}(\tau)
$$

$\mathrm{S}_{\mathrm{T}}(\tau)$ 
VELOCITIES AND EDDY DIFFUSIVITIES FOR PROGRAMS I AND II: LOGARITHMIC VELOCITY DISTRIBUTION, DY $=0.05$

$\mathrm{E}(\mathrm{I})$

$\mathrm{U}(\mathrm{I})$

EA(I)

1

0.00000

0.28500

0.54000

0.76500

0.96000

1. 12500

1. 26000

1. 36500

1. 44000

1.48500

1. 50000

1. 48500

1.44000

1. 36500

1. 26000

1. 12500

0.96000

0.76500

0.54000

0.28500

0.00000

$-2.99573$

$-1.60943$

$-1.08618$

$-0.74638$

$-0.49371$

$-0.29236$

$-0.12491$

0.01843

0.14375

0. 25509

0.35526

0.44629

0.52973

0.60672

0.67821

0. 74493

0.80748

0.86633

0.92191

0.97457

0.14500

0.41500

0.65500

0.86500

1. 04500

1. 19500

1. 31500

1. 40500

1. 46500

1. 49500

1. 49500

1. 46500

1. 40500

1. 31500

1. 19500

1. 04500

0. 86500

0.65500

0.41500

0.14500

SUM

0.00000

20.00000 
PROGRAM FOR CALCULATING $f(t ; x, \eta)$ FOR $\eta=0.50$

FROM PEARSON TYPE III DISTRIBUTION WHEN $\kappa=0.42, \beta=0$

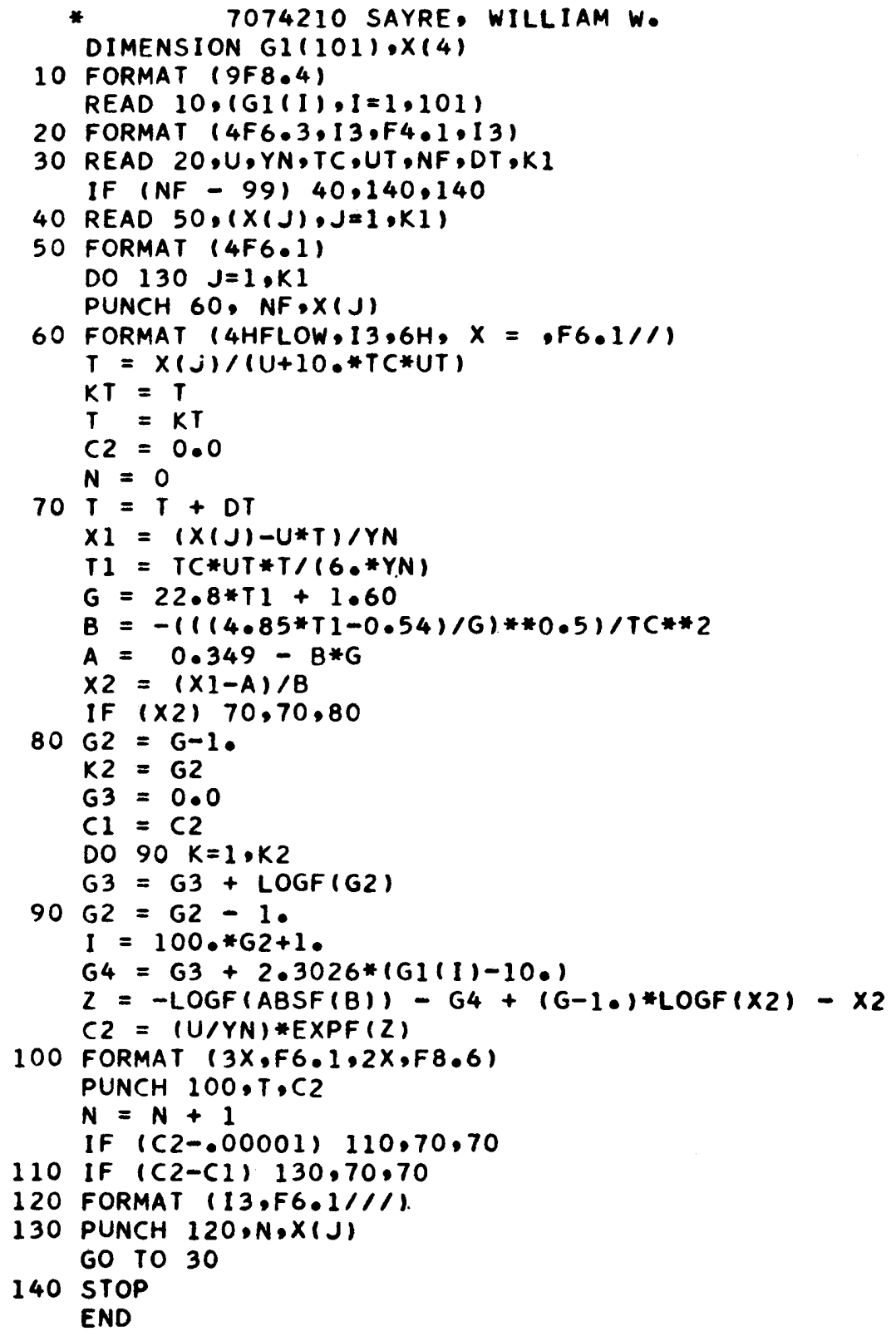




\section{SELECTED VARIABLE NAMES USED IN PROGRAM FOR CALCULATING $f(t ; x, \eta)$ FROM PEARSON TYPE III DISTRIBUTION FUNCTION}

Variable Name

$\mathrm{U}$

YN

TC

UT

NF

DT

K1

$\mathrm{X}(\mathrm{J})$

$\mathrm{T}, \mathrm{KT}$

$\mathrm{N}$

$\mathrm{X} 1$

$\mathrm{T} 1$

G

B

A

$\mathrm{X} 2$

Z

$\mathrm{C} 2$
Term Represented

$\overline{\mathrm{U}}$

$\mathrm{y}_{\mathrm{n}}$

$\kappa$

$\mathrm{U}_{\tau}$

Identification No. for run

$\Delta \mathrm{t}$

No. of curves to be computed

$\mathbf{x}$

No. of points on curve

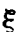

$\tau$

a

b

m

$(\xi-\mathrm{m}) / \mathrm{b}$

$f(\xi ; m, b, a)=f(\xi ; \tau, \eta)$

$\mathrm{f}(\mathrm{t} ; \mathrm{x}, \eta)$ 
Key Words: Diffusion, dispersion, open-channel hydraulics, sediment transport, shear flow, turbulence.

Abstract: The dispersion process for settling particles in a two-dimensional turbulent shear flow is formulated in two differential equations, one for particles suspended in the flow and the other for those deposited on the bed. Exchange of particles between the bed and the flow is permitted. Using the Aris moment trans formations, the equations are converted to a more tractable system of equations which are solved by numerical methods with the aid of a digital computer, for the zero'th, first, second and third moments of the longitudinal concentration distributions. Various combinations of boundary and other input conditions are imposed and their effects on the dispersion process are demonstrated. The results of the numerical solutions together with the results of earlier dispersion experiments with silt particles and fluorescent dyes show that the tendency of the silt particles toward deposition and temporary storage on the bed can profoundly affect the longitudinal dispersion process. Reference: Sayre, William W., Colorado State University Hydrology Paper No. 75,
(August 1975), "Dispers1on of
Key Words: Diffusion, dispersion, open-channel hydraulics, sediment transport shear flow, turbulence.

Abstract: The dispersion process for settling particles in a two-dimensional turbulent shear flow is formulated in two differential equations, one for particles sus pended in the flow and the other for those deposited on the bed. Exchange of

particles between the bed and the flow is permitted. Using the Aris moment trans formations, the equations are converted to a more tractable system of equations which are solved by numerical methods with the aid of a digital computer, for the zero'th, first, second and third moments of the longitudinal concentration distributions. Various combinations of boundary and other input conditions are imposed and their effects on the dispersion process are demonstrated. The results of the numerical solutions together with the results of earlier dispersion experiments with silt particles and fluorescent dyes show that the tendency of the silt particles toward deposition and temporary storage on the bed can profoundly affect the longitudinal dispersion process.

Reference: Sayre, William W., Colorado State University Hydrology Paper No. 75, Sayre, Wil11am W., Colorado State University Hydrology Paper
(August 1975), "Dispersion of Mass In Open-Channel Flow."

Key Words: Diffusion, dispersion, open-channel hydraulics, sediment transport shear flow, turbulence.

Abstract: The dispersion process for settling particles in a two-dimensional turbulent shear flow is formulated in two differential equations, one for particles sus pended in the flow and the other for those deposited on the bed. Exchange of

particles between the bed and the flow is permitted. Using the Aris moment trans particles between the bed and the now is permitted. Using the Aris moment tran formations, the equations are converted to a more tractable system of equations which are solved by numerical methods with the aid of a digital computer, for the zero'th, first, second and third moments of the longitudinal concentration distributions. Various combinations of boundary and other input conditions are imposed and their effects on the dispersion process are demonstrated. The results of the numerical solutions together with the results of earlier dispersion experiments with silt particles and fluorescent dyes show that the tendency of the silt particles toward deposition and temporary storage on the bed can profoundly affect the longitudinal dispersion process.

Reference: Sayre, William W., Colorado State University Hydrology Paper No. 75, (August 1975), "Dispersion of Mass in Open-Channel Flow."
Sayre, Will1am W., Colorado State University Hydrology Paper No. 75 


\section{LIST OF PREVIOUS 25 PAPERS}

No. 50 Probabilities of Observed Droughts, by Jaime Millan and Vujica Yevjevich, June 1971.

No. 51 Amplification Criterion of Gradually Varied, Single Peaked Waves, by John Peter Jolly and Vujica Yevjevich, December 1971.

No. 52 Stochastic Structure of Water Use Time Series, by Jose D. Salas-La Cruz and Vujica Yevjevich, June 1972.

No. 53 Agricultural Response to Hydrologic Drought, by V. J. Bidwell, July 1972.

No. 54 Loss of Information by Discretizing Hydrologic Series, by Mogens Dyhr-Nielsen, October 1972.

No. 55 Drought Impact on Regional Economy, by Jaime Millan, October 1972.

No. 56 Structural Analysis of Hydrologic Time Series, by Vujica Yevjevich, November 1972.

No. 57 Range Analysis for Storage Problems of Periodic-Stochastic Processes, by Jose Salas-La Cruz, November 1972.

No. 58 Applicability of Canonical Correlation in Hydrology, by Padoong Torranin, December 1972.

No. 59 Transposition of Storms, by Vijay Kumar Gupta, December 1972.

No. 60 Response of Karst Aquifers to Recharge, by Walter G. Knisel, December 1972.

No. 61 Drainage Design Based Upon Aeration by Harold R. Duke, June 1973.

No. 62 Techniques for Modeling Reservoir Salinity by John Hendrick, August 1973.

No. 63 Mechanics of Soil Erosion From Overland Flow Generated by Simulated Rainfall by Mustafa Kilinc and Everett V. Richardson, September 1973.

No. 64 Area-Time Structure of the Monthly Precipitation Process by V. Yevjevich and Alan K. Karjlus, August 1973.

No. 65 Almost-Periodic, Stochastic Process of Long-Term Climatic Changes, by William Q. Chin and Vujica Yevjevich, March 1974.

No. 66 Hydrologic Effects of Patch Cutting of Lodgepole Pine, by Thomas L. Dietrich and James R. Meiman, April 1974.

No. 67 Economic Value of Sediment Discharge Data, by Sven Jacobi and Everett V. Richardson, April 1974.

No. 68 Stochastic Analysis of Groundwater Level Time Series in the Western United States, by Albert G. Law, May 1974.

No. 69 Efficient Sequential Optimization in Water Resources, by Thomas E. Croley II, September 1974.

No. 70 Regional Water Exchange for Drought Alleviation, by Kuniyoshi Takeuchi, November 1974.

No. 71 Determination of Urban Watershed Response Time, by E. F. Schulz, December, 1974.

No. 72 Generation of Hydrologic Samples, Case Study of the Great Lakes, by V. Yevjevich May, 1975.

No. 73 Extraction of Information on Inorganic Water Quality, by William L. Lane, August, 1975.

No. 74 Numerical Model of Flow in A Stream-Aquifer System, by Catherine E. Kraeger Rovey, August, 1975. 